Prepared for the U.S. Department of Energy

Under Contract DE-AC05-76RL01830

Technical Letter Report

Assessment of Ultrasonic Phased Array

Testing for Cast Austenitic Stainless Steel

Pressurizer Surge Line Piping Welds and

Thick Section Primary System Cast Piping

Welds

JCN N6398, Task 2A
AA Diaz
KM Denslow
AD Cinson
M Morra
SL Crawford
MS Prowant
SE Cumblidge
MT Anderson

July 2008 


\title{
DISCLAIMER
}

This report was prepared as an account of work sponsored by an agency of the United States Government. Neither the United States Government nor any agency thereof, nor Battelle Memorial Institute, nor any of their employees, makes any warranty, express or implied, or assumes any legal liability or responsibility for the accuracy, completeness, or usefulness of any information, apparatus, product, or process disclosed, or represents that its use would not infringe privately owned rights. Reference herein to any specific commercial product, process, or service by trade name, trademark, manufacturer, or otherwise does not necessarily constitute or imply its endorsement, recommendation, or favoring by the United States Government or any agency thereof, or Battelle Memorial Institute. The views and opinions of authors expressed herein do not necessarily state or reflect those of the United States Government or any agency thereof.

\author{
PACIFIC NORTHWEST NATIONAL LABORATORY \\ operated by \\ BATTELLE \\ for the \\ UNITED STATES DEPARTMENT OF ENERGY \\ under Contract DE-AC05-76RL01830 \\ Printed in the United States of America \\ Available to DOE and DOE contractors from the \\ Office of Scientific and Technical Information, \\ P.O. Box 62, Oak Ridge, TN 37831-0062; \\ ph: (865) 576-8401 \\ fax: (865) 576-5728 \\ email: reports@adonis.osti.gov

\footnotetext{
Available to the public from the National Technical Information Service, U.S. Department of Commerce, 5285 Port Royal Rd., Springfield, VA 22161 ph: (800) 553-6847 fax: (703) 605-6900

email: orders@ntis.fedworld.gov

online ordering: http://www.ntis.gov/ordering.htm
}

This document was printed on recycled paper. 
Technical Letter Report

\section{Assessment of Ultrasonic Phased Array Testing for Cast Austenitic Stainless Steel Pressurizer Surge Line Piping Welds and Thick Section Primary System Cast Piping Welds}

JCN N6398, Task 2A

$\begin{array}{ll}\text { AA Diaz } & \text { KM Denslow } \\ \text { AD Cinson } & \text { M Morra } \\ \text { SL Crawford } & \text { MS Prowant } \\ \text { SE Cumblidge } & \text { MT Anderson }\end{array}$

July 2008

Prepared for the

U.S. Nuclear Regulatory Commission under the U.S. Department of Energy Contract DE-AC05-76RL01830 



\section{Executive Summary}

Research is being conducted for the U.S. Nuclear Regulatory Commission (NRC) at the Pacific Northwest National Laboratory (PNNL) to assess the effectiveness and reliability of advanced nondestructive examination (NDE) methods for the inspection of light water reactor (LWR) components. The scope of this research encompasses primary system pressure boundary materials including cast austenitic stainless steels (CASS), dissimilar metal welds (DMWs), piping with corrosion-resistant cladding, weld overlays, and far-side examinations of austenitic piping welds. A primary objective of this work is to evaluate various NDE methods to assess their ability to detect, localize, and size cracks in coarse-grained steel components. This interim technical letter report (TLR) provides a synopsis of recent investigations at PNNL aimed at evaluating the capabilities of phased-array (PA) ultrasonic testing (UT) methods as applied to the inspection of CASS welds in nuclear reactor piping. A description of progress, recent developments and interim results are provided. The ongoing work presented in this report encompasses:

- The use of phased-array ultrasonic methods and advanced probe designs to enhance detection and characterization of circumferentially oriented flaws in CASS piping specimens, and

- Preliminary investigation of delta-techniques for flaw-depth sizing using tip-diffracted energy from thermal fatigue cracks in CASS components.

While previous work primarily focused on thicker primary loop piping components with outer diameters (OD) ranging from 28 to 36 inches $(71.12$ to $91.44 \mathrm{~cm}$ ), a variety of thinner cast piping welds exist in pressurized water reactors (PWRs) and an optimum frequency has yet to be established to examine these thin CASS components. While the sample specimens used in this study may not represent worst-case field conditions with regard to microstructures, weld root conditions, counterbore facets, surface roughness/geometry, etc., the sample specimens provide a range of grain structure conditions and are representative of components found in plants.

The work reported here is focused toward evaluating UT inspection capabilities in CASS pressurizer surge line piping welds as well as continuing to assess improved PA probe designs on primary loop, CASS piping. PNNL was tasked with acquisition and procurement of materials, fabrication of flawed specimens, modeling and procurement of specialized phased-array probes, and conducting data acquisition and analysis on these specimens. The macrostructure of vintage pressurizer (PZR) surge line piping to determine grain size, shape, and orientation was performed in FY-2007. The material comprising these components include both centrifugally cast (pipe segments) and statically cast (elbows), with dimensions of approximately 12 - to 14 -inch $(30.5$ to $35.5 \mathrm{~cm})$ OD and 1.3 -inch $(33-\mathrm{mm})$ wall thickness. This thickness is representative of typical PZR surge lines and various safe-ends in Combustion Engineering-designed plants. The ongoing activities reported here included PA-UT examinations on implanted/fabricated thermal fatigue cracks to evaluate detection, localization, and sizing performance as a function of frequency. In conjunction with these activities, PNNL employed customdesigned, low-frequency PA probes for continued evaluation of inspection performance on thicker-section CASS pipe segments, such as the Westinghouse Owner's Group practice set of specimens. 
The work consisted of design and sound field modeling for various PA probes and probe configurations, development of specific PA focal laws, and use of a new data acquisition system for implementing examinations, including:

- Low-frequency PA-UT examination of CASS PZR surge-line components

- As a function of inspection frequency $(1.5 \mathrm{MHz}$ and $800 \mathrm{kHz})$

- Low-frequency PA-UT examination of thick-section, primary loop CASS piping components

- New data acquired at $800 \mathrm{kHz}$, with an optimized PA system

- Initial evaluation of the PA-delta technique for detecting tip-diffracted energy in CASS

- Contrasting data from examinations in wrought stainless parent material with electrical discharge machining (EDM) notches at 10\%, 20\%, 30\%, and 40\% through-wall depth versus thermal fatigue crack tips in the CASS PZR surge-line specimen.

Low-frequency phased-array and delta-technique data were acquired on specimens in the 12-14 inch (305-356 mm) diameter range using a manual-encoded scanner while data acquired on the larger diameter pipe sections ( 32 inch $[813 \mathrm{~mm}]$ ) were acquired with a flat track scanner. Data acquisition was accomplished using a prototype, low frequency DYNARAY ${ }^{\circledR}$ system for the $800-\mathrm{kHz}$ probe that accommodates a maximum of 128 channels and runs beta-version Ultravision ${ }^{\mathbb{R}} 3.0 \mathrm{~B} 1$ software. Its lowfrequency pulsing will drive probes in the $0.2-20 \mathrm{MHz}$ range. The $1.5-\mathrm{MHz}$ data were acquired with a standard Tomoscan ${ }^{\circledR}$ III system, which has 32 channels in transmission and up to 64 channels in the receive mode, and runs Ultravision ${ }^{\circledR} 1.1 \mathrm{Q} 5$ software. This system was designed to drive probes in the 1-25 MHz range. For the delta-technique, the setup included configuring two $800-\mathrm{kHz}$ phased array probes in an arrangement similar to that of a time-of-flight diffraction (TOFD) method, where transmit and receive transducers are oriented opposite and facing each other, with some separation distance.

Results show that the thermal fatigue cracks in the PZR surge line specimen were detected from both the centrifugally cast stainless steel (CCSS) and the statically cast stainless steel (SCSS) sides of the weld at $800 \mathrm{kHz}$ and at $1.5 \mathrm{MHz}$. Length sizing was better from the SCSS side, with a factor of two less error in the $800-\mathrm{kHz}$ data and only slightly less error in the $1.5-\mathrm{MHz}$ data as compared to the CCSS side. The crack depth sizing was similar from either the CCSS or the SCSS side at both frequencies with an approximate 3-mm error. Signal-to-noise values were in the 6.8 to $23 \mathrm{~dB}$ range showing generally good detection of both the crack corner signal and top of flaw signal.

The $800-\mathrm{kHz}$ probe with a wavelength in the material of approximately $7 \mathrm{~mm}(0.28 \mathrm{inch})$ performed well in comparison to the $1.5-\mathrm{MHz}$ probe, which has a wavelength of $4 \mathrm{~mm}(0.16 \mathrm{inch})$. A very limited frequency analysis showed that the $800-\mathrm{kHz}$ probe was actually operating at $730-780 \mathrm{kHz}$ in the material due to microstructural "filtering" effects, and the 1.5-MHz probe was effectively operating at 1-1.2 MHz. With both probes approaching a similar frequency range of operation in this thinner material and for these four flaws, it is not surprising that the signal-to-noise ratio (SNR) and depth sizing results were similar. Length sizing differences were attributed to variations in the effective focal spot size of each phased-array beam. It should be noted that the microstructures for both the elbow and pipe sections of the PZR surgeline were comprised of finer grained microstructures where the average grain diameters were typically smaller than the wavelength of the ultrasonic energy used to inspect this specimen. The PZR surge-line specimen evaluated here represents only one type of microstructure, and additional work using the $800-\mathrm{kHz}$ PA probe will be needed to more effectively assess detection and sizing capabilities for PZR 
surge-line components where the average grain diameters are much larger and where material homogeneity and grain anisotropy will provide more significant inspection challenges.

The limited data comparison from the three WOG specimens evaluated here shows that the $800-\mathrm{kHz}$ probe has the potential for inspecting some of the thicker section coarse-grained materials where attenuation and scattering allow for suitable penetration and resolution. However, it is anticipated that a lower frequency $(500 \mathrm{kHz})$ will be required for many of the dimensionally larger microstructures exhibited (as an example) by the more challenging WOG practice set of specimens that were not evaluated here. The $800-\mathrm{kHz}$ probe was pulsed at its optimum frequency, an 825-nanosecond pulse in this instance. In both the MPE-6 and OPE-5 specimens the center frequency of the flaw signal was greater on the SCSS side than the CCSS side showing that this CCSS material attenuates and scatters more than the SCSS material and also contributes to frequency "filtering" effects. The signal frequency dropped from 810 to $780 \mathrm{kHz}$ in the MPE-6 specimen and from 780 to $680 \mathrm{kHz}$ in the OPE-5 specimen. Again, these are preliminary findings are based on limited data.

With regard to acquiring baseline data and evaluating the delta-technique for capturing tip-diffracted energy from crack tips in CASS, the 40\%,30\%, and 20\% through-wall EDM notches were detected and depth sized with a root mean square error (RMSE) of $2.29 \mathrm{~mm}(0.091 \mathrm{inch})$ and length sized with a RMSE of $7.62 \mathrm{~mm}(0.30 \mathrm{inch})$ in the wrought stainless steel specimens. The $10 \%$ through-wall EDM notch was not detected using the delta technique. This is attributed to the size of the wavelength relative to the size of the notch.

Flaws 1, 2, and 4 on the PZR surge-line specimen were detected and depth sized with an RMSE of $3.44 \mathrm{~mm}(0.116 \mathrm{inch})$ and length sized with an RMSE of $24.0 \mathrm{~mm}$ (0.812 inch). Flaw 3 was not scanned using the delta-technique. The flaw sizing tended to be slightly larger than the true state. This "resolution" issue is attributed to the blooming effect that occurs from an incident sound field where the large $7.5-\mathrm{mm}$ wavelength in the material relative to the flaw size results in a distortion of the scattered energy where the resultant image has an exaggerated size, and hence oversized dimension.

The tip-diffraction signals observed from the EDM notches have low to moderate signal-to-noise ratios that range from $1.62(4.2 \mathrm{~dB})$ to $3.70(11.4 \mathrm{~dB})$. The certainty in reporting tip-diffraction signals comes from the significant backwall signal dropout that accompanies these low-amplitude responses, which range from $3.26(10.3 \mathrm{~dB})$ to $8.23(18.3 \mathrm{~dB})$. If the backwall dropout were not evident in the overall images, it would be more challenging to identify and discriminate the tip-diffracted energy from coherent energy scattered from the grain boundaries. As anticipated, the larger the EDM notch (in through-wall depth) the larger the SNR of the tip-diffracted signal for the case of the wrought stainless steel (WSS) coupons. The, tip-diffracted signals observed from the thermal fatigue cracks in the pressurizer surge line were similar to their EDM counterparts, with the exception of Flaw \#2, with signalto-noise ratios ranging from $2.6(8.3 \mathrm{~dB})$ to $12(21.6 \mathrm{~dB})$. The detection and localization of crack tipdiffracted signals is again only made possible from observing a significant backwall signal loss that accompanies these signals, which range from $7.98(18.0 \mathrm{~dB})$ to $12.0(21.6 \mathrm{~dB})$.

The frequency and focusing/steering of the phased-array probes used in these delta-technique examinations were not designed to accommodate very thin sample materials. Nevertheless, the results obtained in this study demonstrate that there is potential for improving the delta technique by employing optimally designed probes and suitably chosen inspection frequencies. The degree of success, however, is contingent upon the appropriate phased-array probe designs, the amount of background scatter from the microstructure, and the ability to capture the backwall signal dropout as a fiduciary for locating/detecting 
the areas where tip-diffracted energy should be seen. With suitable probes designed for thin materials and more evaluation of appropriate focal laws, the delta-technique approach may prove useful for crack depth determination. 


\section{Acronyms and Abbreviations}

\begin{tabular}{|c|c|}
\hline ASME & American Society of Mechanical Engineers \\
\hline BMI & bottom-mounted instrumentation \\
\hline BWR & boiling water reactor \\
\hline CASS & cast austenitic stainless steel \\
\hline CRDM & control rod drive mechanism \\
\hline CCSS & centrifugally cast stainless steel \\
\hline DMW & dissimilar metal weld \\
\hline EDM & electrical discharge machining \\
\hline EPRI & Electric Power Research Institute \\
\hline ET & eddy current testing \\
\hline ID & inner diameter \\
\hline ISI & inservice inspection \\
\hline LWR & light water reactor \\
\hline NDE & nondestructive examination \\
\hline NRC & U.S. Nuclear Regulatory Commission \\
\hline OD & outer diameter \\
\hline PA & phased array \\
\hline PA-UT & phased array ultrasonic testing \\
\hline PNNL & Pacific Northwest National Laboratory \\
\hline PZR & pressurizer \\
\hline PWR & pressurized water reactor \\
\hline RMSE & root mean square error \\
\hline RPV & reactor pressure vessel \\
\hline SAFT & synthetic aperture focusing technique \\
\hline SCSS & statically cast stainless steel \\
\hline SG & steam generator \\
\hline SNR & signal-to-noise ratio \\
\hline TLR & technical letter report \\
\hline TOFD & time-of-flight diffraction \\
\hline TRL & transmit-receive longitudinal \\
\hline UT & ultrasonic testing \\
\hline WOG & Westinghouse Owner's Group \\
\hline WSS & wrought stainless steel \\
\hline
\end{tabular}





\section{Contents}

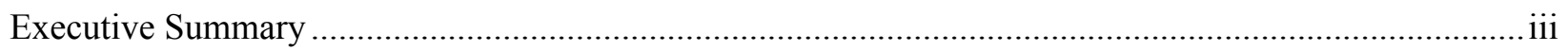

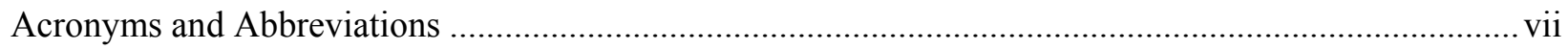

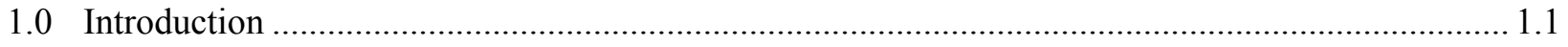

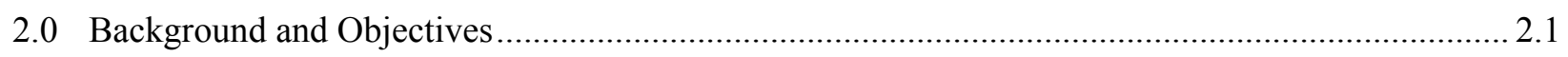

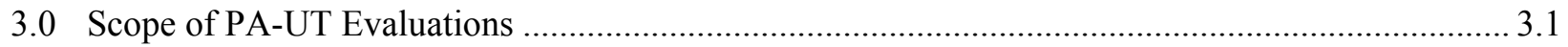

4.0 The CASS Inspection Challenge ............................................................................................ 4.1

5.0 Phased Array Probes, Modeling, and Focal Law Development .................................................... 5.1

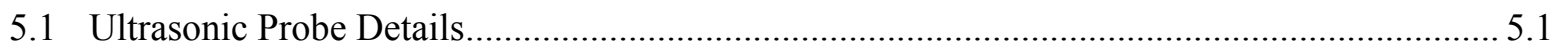

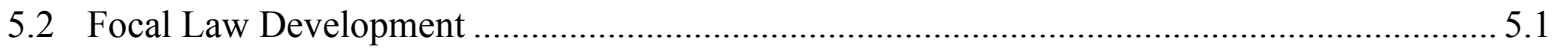

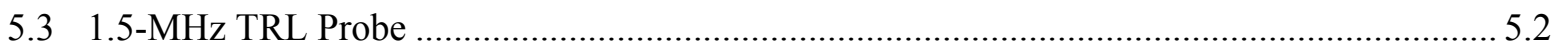

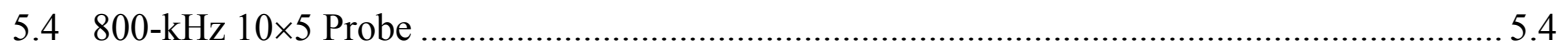

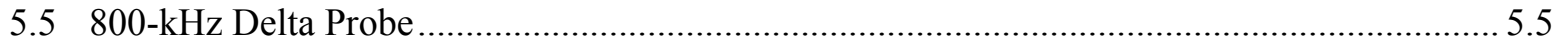

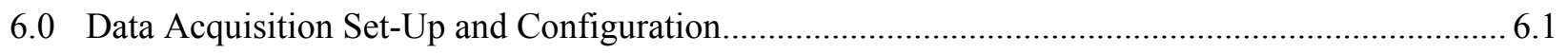

6.1 Phase Array Data Acquisition (PZR Surge Line and WOG Specimens) .............................. 6.1

$6.2800-\mathrm{kHz}$ Delta-Technique Data Acquisition ......................................................................... 6.2

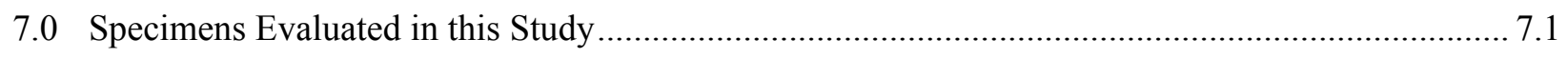

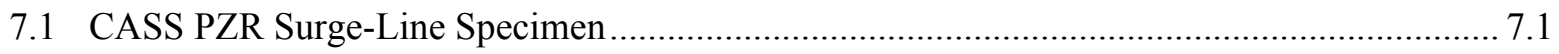

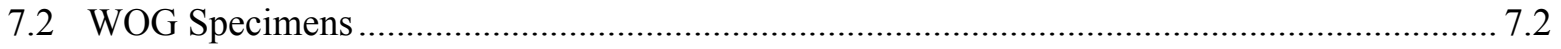

7.3 Wrought Steel Coupons with EDM Notches .................................................................. 7.4

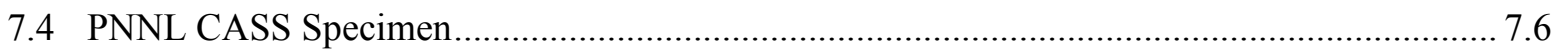

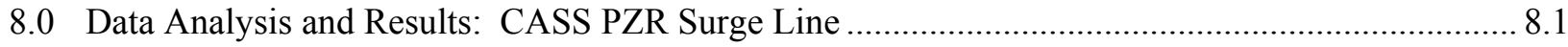

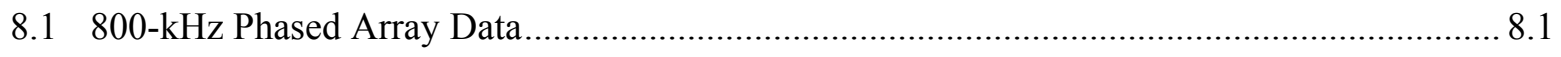

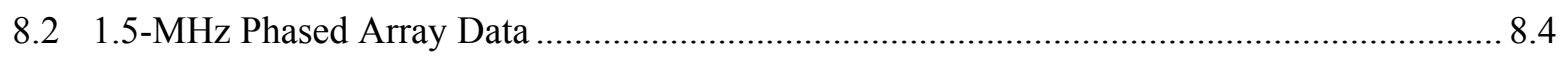

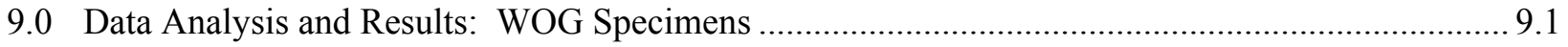

10.0 Data Analysis and Results: Delta-Technique (Tip Diffraction) …........................................... 10.1

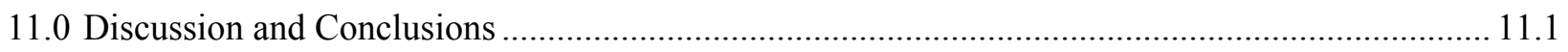

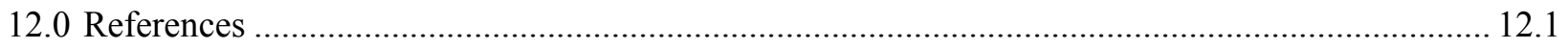

Appendix A 800-kHz Phased Array Data on the Pressurizer Surge Line .............................................. A.1

Appendix B 1.5-MHz Phased Array Data on the Pressurizer Surge Line ................................................. 1

Appendix C WOG Data with the 800-kHz Phased Array Probe ……....................................................

Appendix D 0.8-MHz Phased Array Data for Delta-Technique Evaluation......................................... D.1 


\section{Figures}

4.1 Photographs of Polished and Chemically Etched CASS PZR Ring Segment Showing the Pipe-Side Grain Microstructure and the Elbow-Side (SCSS) Grain Microstructure...................... 4.2

4.2 Digital Photographs of Coarse-Grained Microstructures in a PZR Surge Line at PNNL .............. 4.3

5.1 The ZETEC Advanced Phased Array Calculator is Useful for Generating Focal Laws and Simulating the Sound Field for the Focal Law to Determine Beam Characteristics ...................... 5.2

5.2 1.5-MHz TRL Probe Originally Designed for Wrought Stainless Steel Welds ............................ 5.3

5.3 Simulations of the Beams Created by the 1.5-MHz TRL Arrangement for $30^{\circ}, 45^{\circ}$, and $60^{\circ} \ldots \ldots . .5 .3$

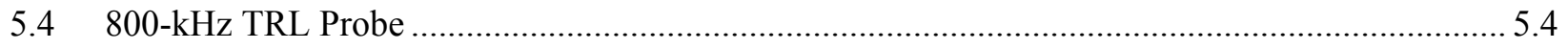

5.5 Simulations of the Beams Created by the $800-\mathrm{kHz}$ TRL Arrangement for $30^{\circ}, 45^{\circ}$, and $60^{\circ} \ldots \ldots . .5 .5$

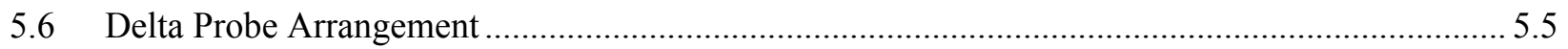

5.7 Simulated Sound Field for the $10 \times 5$ Transmitter at $55^{\circ}$ for Delta-Technique Evaluations ............ 5.6

5.8 Simulated Sound Fields for the Receiver Focused 0.5 Inches under the Front of the Wedge......... 5.7

5.9 Simulated Sound Fields for the Transmitter Focused 0.5 Inch in Front of the Wedge .................. 5.7

5.10 Simulated Sound Fields for the Receiver Focused 0.5 Inch in Front of the Wedge....................... 5.8

6.1 Photographs of the PZR Surge Line Specimen .................................................................. 6.1

6.2 Top and Front Views of the Immersion Tub Scanning Configuration for the PNNL CASS Specimen Using an Automated X-Y Axis, Flat Peg-Scanner and Spring-Loaded Gimbal

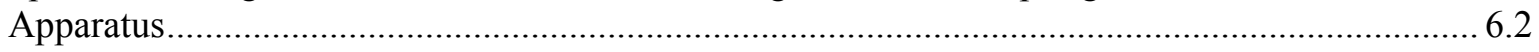

6.3 Photograph of Delta-Technique Configuration Just Prior to Scanning ….................................... 6.3

6.4 Top and Side Views of the Delta-Technique Chassis Mounted on a PNNL CASS Specimen for Data Acquisition, Using the Automated Peg-Scanner in an Immersion Tub ............................ 6.4

7.1 Photographs of the inside surface of the CASS PZR surge-lLine after excavation and preparation of the areas targeted for flaw implantation......................................................... 7.1

7.2 Photographs of the Three WOG Specimens Examined -WOG specimen MPE-6; WOG

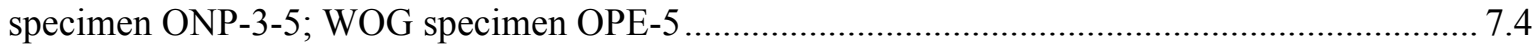

7.3 Photographs of the WSS Parent Material from which the Two Calibration Coupons were

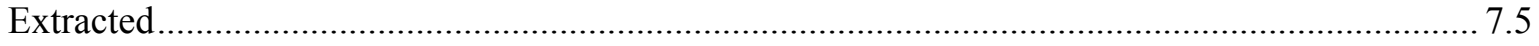

7.4 Photographs of the End View and ID Surface View for One of the WSS Coupons with EDM Notches, Used for Evaluation and Baseline Measurements of the PA Delta-

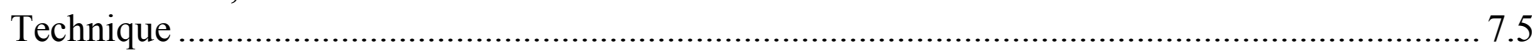

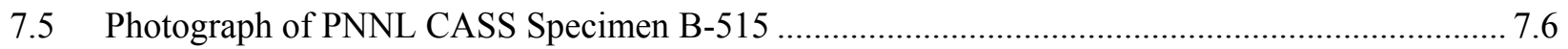

8.1 PZR Surge Line Flaw 1 from the CCSS Pipe Side at an Axial Position of $-130 \mathrm{~mm}$ and at

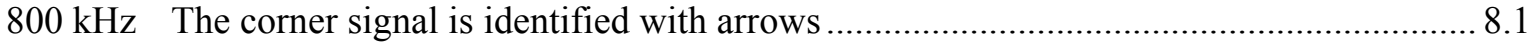

8.2 PZR Surge Line Flaw 1 from the CCSS Pipe Side at an Axial Position of $-130 \mathrm{~mm}$................... 8.2

8.3 A Strong Corner Response from Flaw 4 is Shown in the Data and is More Typical of Flaw Responses Flaw 3 has a strong top of flaw signal and exhibits a weaker corner signal

9.1 Thermal Fatigue Crack is Detected in MPE-6; 800-kHz Data from the SCSS Side of the Weld

10.1 Example of Probes Positioned Equidistant from Weld Centerline or Flaw ............................... 10.1 
10.2 Example of Receiver Positioned Slightly Closer to the Weld Centerline or Flaw ...................... 10.1

10.3 Example of Receiver Positioned Directly Over the Weld Centerline or Flaw ............................ 10.2

10.4 End-view from the Wrought Stainless Steel 40\% EDM Notch Scan ......................................... 10.3

10.5 Notch Length Determination from the Wrought Stainless Steel 40\% EDM Notch Data as Determined by Loss of Backwall Signal ............................................................................. 10.3

10.6 Measured Flaw Lengths Versus True Flaw Lengths as a Function of Flaw Number on the PZR Surge-Line Specimen Using All Frequencies and Techniques.

10.7 Measured Flaw Depths Versus True Flaw Depths as a Function of Flaw Number on the PZR Surge-Line Specimen Using All Frequencies and Techniques 10.6 


\section{Tables}

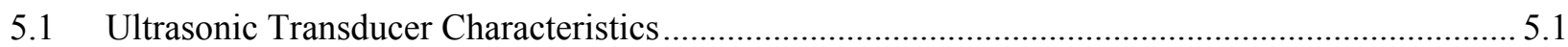

7.1 Positional and Dimensional Data for Implanted Flaws in the CASS PZR Surge Line .................. 7.2

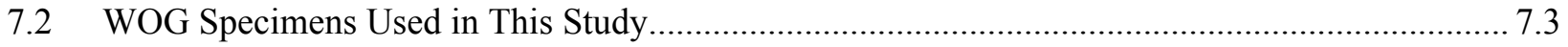

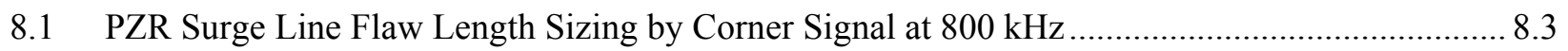

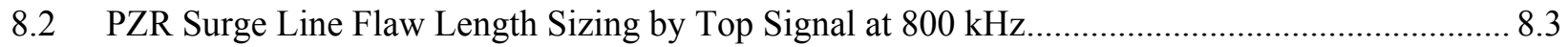

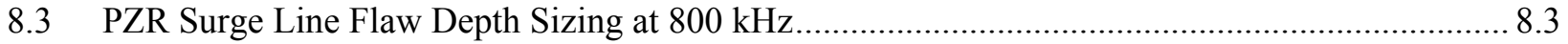

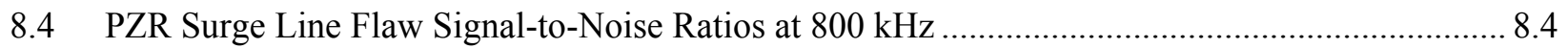

8.5 PZR Surge Line Flaw Length Sizing by Corner Signal at $1.5 \mathrm{MHz}$..................................... 8.5

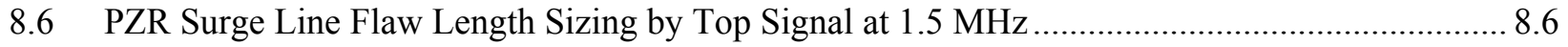

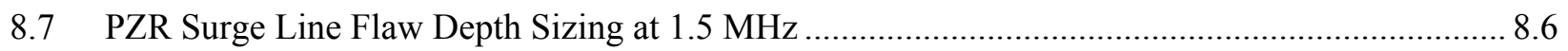

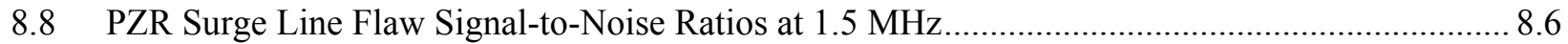

9.1 Length Sizing of WOG Specimens with Phased Array at Various Inspection Frequencies ........... 9.2

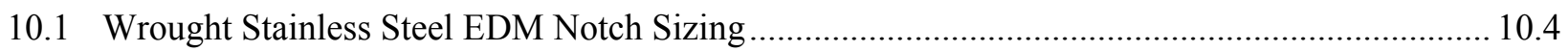

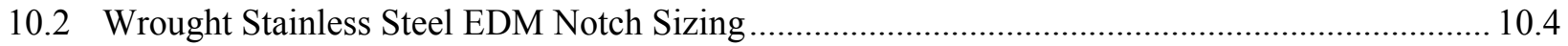

10.3 Wrought Stainless Steel EDM Signal-to-Noise Ratios .......................................................... 10.4

10.4 Pressurizer Surge Line Thermal Fatigue Crack Sizing.............................................................. 10.5

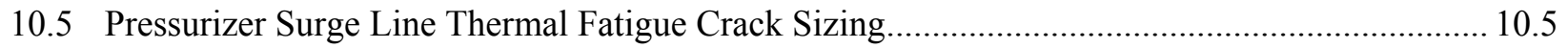

10.6 Pressurizer Surge Line Signal-to-Noise Ratios .................................................................... 10.5 


\subsection{Introduction}

This document is a technical letter report summarizing recent laboratory evaluations of phased array (PA) ultrasonic testing (UT) on cast austenitic stainless steel (CASS) pressurizer (PZR) surge line piping, and thick section primary loop cast piping welds as performed at the Pacific Northwest National Laboratory (PNNL) in Richland, Washington. Work reported here was conducted as part of a U.S. Nuclear Regulatory Commission (NRC) project at PNNL entitled, "Reliability of Nondestructive Examination for Nuclear Power Plant Inservice Inspection," under Task 2 of JCN N6398. This technical letter report (TLR) is submitted as an Operating Plan Milestone deliverable for Task 2.

The focus of the research is to determine the effectiveness and reliability of advanced nondestructive examination (NDE) methods on light water reactor components containing cast stainless steel material and other coarse-grained components that encompass dissimilar metal welds (DMWs), piping with corrosion-resistant cladding, weld overlays, and far-side examinations of austenitic piping welds. The specific goal of this work is to assess the effectiveness and reliability of various NDE methods to detect and, if possible, size cracks in coarse-grained steel components.

The work described here was performed to provide insights for evaluating the utility of advanced NDE approaches for the inspection of CASS components. This document is intended as an interim technical letter report to describe recent findings and provide a detailed technical status of on-going activities. Section 2 of this report describes the project background and technical objectives. Section 3 provides a description of the scope of work as it pertains to this evaluation for both PA inspection capability and evaluation of a delta-technique for detection of tip-diffracted energy from flaws in CASS materials. Section 4 provides a discussion of the CASS inspection problem as it relates to the propagation of ultrasonic energy in coarse grained microstructures. Section 5 describes the various probes and probe configurations used, modeling of the probe configurations to generate expected performance information, and issues associated with PA focal law development. Section 6 provides a description of the data acquisition set-up and experimental approach employed in this work, while Section 7 describes the various CASS specimens used in these laboratory studies. Sections 8, 9, and 10 provide discussions of the data analysis process and results from PA examinations of the CASS PZR surge line, some thicksection CASS Westinghouse Owner's Group (WOG) specimens, wrought steel coupons with electrical discharge machined (EDM) notches for tip-diffraction studies, and a PNNL CASS pipe segment, respectively. Section 11 includes a technical discussion and conclusions from the work presented here. Section 12 addresses unresolved issues and a description of work currently in progress, while Section 13 identifies references cited in this TLR. 



\subsection{Background and Objectives}

Since 1977, PNNL has conducted research under NRC guidance to evaluate state-of-the-art technical approaches for inspecting coarse-grained steel reactor components (Taylor 1984; Diaz et al. 1998; Anderson et al. 2007). This work recently has focused on assessing the viability of phased array ultrasonic NDE methods applied from the outside pipe surface of the components. Regarding ultrasonic methods, PNNL's strategy has been to evaluate low-frequency (500 kHz to $1.5 \mathrm{MHz}$ ) phased-array ultrasonic testing (PA-UT) methods to assess the viability in using long-wavelength (low-frequency) inspection techniques that are inherently less sensitive to the effects of the coarse-grained microstructure, while maintaining suitable resolution to effectively detect, localize, and size flaws in these materials (Anderson et al. 2003; Anderson et al. 2007).

The main objectives of Task 2 in JCN N6398 are focused on assessing the effectiveness of new techniques and methods that allow for the consistent and reliable inspection of coarse-grained materials having complex geometrical conditions. PNNL is tasked with determining if certain NDE techniques (e.g., phased-array UT, synthetic aperture focusing technique [SAFT] UT) can reliably and effectively detect and characterize flaws on the far side of austenitic welds, the far side of dissimilar metal welds, and either side of CASS components; and identify problem areas that have resulted in the failure of NDE methods to detect flaws in areas such as control rod drive housings. This includes inspection applications where there are components with corrosion-resistant cladding and weld overlays as well. These techniques should allow for discrimination between coherent ultrasonic energy scattered from grain boundaries (metallurgical or geometric reflectors) and cracks (e.g., discontinuities) in coarse-grained steel components. New piping materials and joining processes are being developed and pursued by the industry (e.g., polyethylene pipe) and inspection reliability for these materials is also being addressed in this task.

In early FY-2007, the NRC published NUREG/CR-6933, Assessment of Crack Detection in HeavyWalled Cast Stainless Steel Piping Welds Using Advanced Low-Frequency Ultrasonic Methods (Anderson et al. 2007), which provided a detailed description of the inspection challenges posed by CASS as it relates to the microstructures and component geometries of primary piping loop configurations. This NUREG/CR documented PNNL's initial results of using a new 500-kHz phased array approach to examine pressurized water reactor (PWR) primary coolant system piping welds. These welds are in cast piping of approximately 3 -inch $(76.2-\mathrm{mm})$ thick, where a frequency of $500 \mathrm{kHz}$ was necessary to penetrate this material thickness with reduced attenuation. However, thinner cast piping welds exist at PWRs, such as on PZR surge lines and safe-ends to dissimilar metal welds, and an optimum frequency has yet to be established to examine relatively thin CASS components. In early FY-2008, PNNL generated a letter report documenting results from an initial investigation of low-frequency PA-UT methods for use on these thinner piping welds. This letter report provided a status of technical activities that included initial ultrasonic experiments on machined reflectors to ascertain the optimal center frequency for sound propagation and crack detection in these materials.

Part of this previous work focused on acquiring baseline PA-UT data using a 1.5-MHz phased array probe to image the end-of-block on both the centrifugally cast stainless steel (CCSS) pipe-side and the statically cast stainless steel (SCSS) elbow side of a PZR surge-line weld obtained from a cancelled nuclear power plant. The pipe-to-elbow weld volume was also imaged using this probe to obtain PA-UT 
data from the weld prior to implantation of thermal fatigue cracks. These data were used to evaluate the effectiveness of this frequency range for suitable penetration and resolution in these components, and subsequently provided the basis for additional design and modeling of a more effective PA probe operating at a lower frequency $(800 \mathrm{kHz})$. At the end of this initial study, PNNL defined the specifications for implantation of four thermal fatigue cracks in the austenitic weld of this CASS PZR surge-line for further evaluation of flaw detection, localization, and sizing capabilities with this technique; the results of which are reported here. 


\subsection{Scope of PA-UT Evaluations}

The overall NRC project under which this work was performed addresses a range of NDE issues associated with coarse-grained steels and challenging material/component configurations (e.g., CASS, DMWs, Alloy 600/182/82). Work is focused on assessing the capabilities of state-of-the-art phased array ultrasonic testing, advanced low-frequency/SAFT UT methods, and eddy current testing (ET) techniques for effective and reliable inspection of cast components; far-side inspections on wrought stainless steel (WSS) piping with austenitic welds (in both boiling water reactor [BWR] and PWR configurations); vessel penetrations (control rod drive mechanism [CRDM] nozzles, bottom-mounted instrumentation [BMI] nozzles, and PZR heater sleeves; and DMWs associated with reactor pressure vessel/steam generators (RPV/SG) and PZR nozzle-to-pipe welds. Concurrently, a concentrated effort to assess casting processes for selected components to better understand the effects of these processes on microstructure and variability from component to component will provide a technical basis for optimization of UT probe designs.

The scope of the work reported here was defined by NRC guidance and is focused toward evaluating UT inspection capabilities in CASS pressurizer surge line piping welds as well as continuing evaluation on thicker-section primary loop, CASS piping. PNNL was tasked with acquisition and procurement of materials, fabrication of flawed specimens, modeling and procurement of specialized phased-array probes, and conducting data acquisition and analysis on these specimens. Mapping the macrostructure of vintage pressurizer surge line piping to determine grain size, shape, and orientation is also included, and was performed in FY-2007. The material comprising these components include both centrifugally cast (pipe segments) and statically cast (elbows) microstructures, where the PZR surge-line component has dimensions of approximately 12 - to 14 -inch $(30.5$ to $35.5 \mathrm{~cm})$ outer diameter (OD) with a 1.3-inch (33-mm) wall thickness. This thickness is representative of typical PZR surge lines and various safe-ends in Combustion Engineering-designed plants. PNNL's efforts include performing PA-UT examinations on implanted/ fabricated thermal fatigue cracks and evaluating detection, localization, and sizing performance as a function of frequency. In conjunction with these activities, PNNL is employing customdesigned, low-frequency PA probes for continued evaluation of inspection performance on thicker-section CASS pipe segments, such as the Westinghouse Owner's Group practice set of specimens. The scope of the work reported here also includes an initial assessment of novel ultrasonic techniques; for example, delta technique for detection of tip-diffracted energy for depth-sizing of cracks in CASS. Thus, the overall scope of the work reported in this TLR includes:

- Low-frequency PA-UT examination of CASS PZR surge-line components

- As a function of inspection frequency $(1.5 \mathrm{MHz}$ and $800 \mathrm{kHz})$

- Low-frequency PA-UT examination of thick-section, primary loop CASS piping components

- New data acquired at $800 \mathrm{kHz}$, with an optimized PA system

- Initial evaluation of the PA-delta technique for detecting tip-diffracted energy in CASS

- Contrasting data from examinations in wrought stainless parent material with EDM notches at $10 \%, 20 \%, 30 \%$, and $40 \%$ through-wall depth versus thermal fatigue crack tips in the CASS PZR surge-line specimen. 



\subsection{The CASS Inspection Challenge}

The relatively low cost and high-corrosion resistance of cast stainless steel has resulted in extensive use of this material in the primary coolant piping systems of Westinghouse-designed PWRs (Taylor 1984). Alloying elements and casting processes used in the fabrication of CASS materials are responsible for its corrosion resistance and strength but also create complex and coarse-grained microstructures. This material is anisotropic and inhomogeneous. The manufacturing process can result in the formation of columnar (dendritic) grain structures often several centimeters in length, with grain growth oriented along the direction of heat dissipation, typically normal to the surface. Additionally, during the cooling and solidification process, columnar, equiaxed (randomly speckled microstructure), or a mixed structure can result, depending on chemical content, control of the cooling, and other variables in the casting process (Taylor 1984; Jeong 1987). The OD and inner-diameter (ID) surfaces of specimens used in the current study possess relatively smooth, machined conditions; this is a normal part of the fabrication method, performed to remove imperfections resulting from the casting process.

CASS piping is subject to periodic UT based on requirements found in the American Society of Mechanical Engineers (ASME) Boiler and Pressure Vessel Code, Section XI, Rules for Inservice Inspection (ISI) of Nuclear Power Plant Components (Examination Category B-J, Pressure Retaining Welds in Piping). For ISI to be successful, service-induced flaws must be detected and repaired prior to becoming of such size that the integrity of a component is compromised. Flaw detection is the initial priority, and for UT this is accomplished by analyzing ultrasonic echo waveforms from reflections within the volume of inspected material that are potentially caused by service degradation. The large size of the anisotropic grains relative to the acoustic pulse wavelength severely scatters and attenuates the sound field. Significant changes in acoustic velocity are also evident. Refraction and reflection of the sound beam occur at the grain boundaries, effectively skewing and/or partitioning the sound field, resulting in flaws being incorrectly identified, specific volumes of material not being examined, or both. To reduce the impact of the microstructure on the inspection technique, the work reported here focuses on lowfrequency ( $800 \mathrm{kHz}$ to $1.5 \mathrm{MHz}$ ) sound field propagation through the material as applied from the OD surface. The PZR surge line specimen used in this study consists of a CCSS pipe segment welded to a SCSS elbow segment. Rings from both ends were cut, polished, and etched to show the microstructures of the pipe and elbow ends (equiaxed and columnar, respectively) as shown in Figure 4.1.

False colors have been added to assist the viewer in judging microstructural variation and banding. Typical grain diameters range in size from approximately 0.04 inch $(1 \mathrm{~mm})$ to nearly $0.25 \mathrm{inch}(6.35 \mathrm{~mm})$ or more as a function of depth or spatial position in the material. At a $1.5-\mathrm{MHz}$ inspection frequency, the corresponding wavelength in the CASS PZR surge-line material is approximately 0.15 inch $(3.8 \mathrm{~mm})$. Thus, the wavelength at this inspection frequency will experience more scattering and more attenuation as the sound field propagates through the material. In contrast, at an $800-\mathrm{kHz}$ inspection frequency, the wavelength in the material is approximately 0.28 inch $(7.1 \mathrm{~mm})$. The sound field for the lower inspection frequency will be less sensitive to the effects of grain boundary scattering and attenuation as the wavelength is generally larger than the majority of the grains in the microstructure for this specimen. 

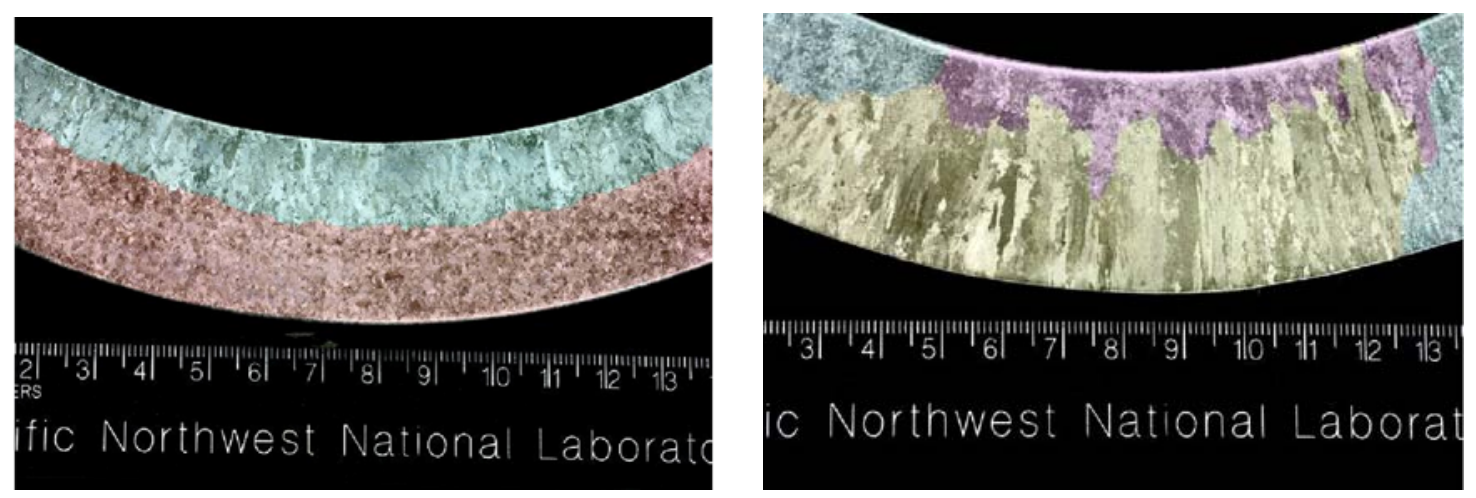

Figure 4.1. Photographs of Polished and Chemically Etched CASS PZR Ring Segment Showing the Pipe-Side (CCSS) Grain Microstructure (left) and the Elbow-Side (SCSS) Grain Microstructure (right)

While the PZR surge-line specimen evaluated and reported on here consisted of dimensionally smaller grains, some PZR surge-line microstructures exhibit larger average grain diameters. PNNL obtained a long segment of a PZR surge line that was sectioned on both sides of the elbow. These sections were sliced, polished, and chemically etched to bring out the microstructures of both pipe segments on opposite sides of the elbow joint shown in Figure 4.2. The resultant micrographs showed the variability and range of grain sizes that can be found in PZR surge-line components. Larger columnar grains that appear as "blocks of material" are evident on one side of the elbow, while thinner dendritic grains make up the pipe's microstructure on the other side of the weld. In this case, the average grain diameters and overall microstructures of these segments would provide a more challenging inspection scenario.

In contrast to the smaller diameter PZR surge-line piping, primary piping components with thicker wall dimensions and larger diameters provide even more significant challenges to ultrasonic inspections. The cumulative average size of grains in varied regions of these materials has been measured previously in a number of CASS specimens using a lineal mean intercept method (Anderson et al. 2007; Diaz et al. 2007). However, this average is not a reliable indicator of overall dominant grain sizes and distributions for the WOG specimens used in this study. Previous work in characterizing average grain size for CASS materials in large-bore piping indicates that, in general, grains in the range of $0.47-0.59$ inch $(12-15 \mathrm{~mm})$ in diameter are found for SCSS microstructures (cast elbows), while 0.67-0.79 inch (17-20 mm) grains are typical for CCSS microstructures (cast piping). Thus, the tradeoff between effective penetration and required resolution for flaw detection becomes an issue, as the better inspection frequencies will typically be found at wavelengths greater than the average grain diameter. In the case of the WOG specimens evaluated in this study, suitable inspection frequencies will lie in the $300-\mathrm{kHz}$ to $500-\mathrm{kHz}$ range, which corresponds to wavelengths of approximately $0.75 \mathrm{inch}(19.1 \mathrm{~mm})$ to $0.45 \mathrm{inch}(11.4 \mathrm{~mm})$, respectively. Advanced signal processing and image reconstruction algorithms (SAFT, holographic techniques, etc.) can typically provide flaw images with resolution of approximately a half wavelength $(\lambda / 2)$. It is worthy to note that some specimens used in this study also contain wrought stainless steel, where the fine microstructure is made up of randomly distributed equiaxed grains of very small dimension (less than 0.5 -mm diameter) relative to the grain diameters found in CASS microstructures. 


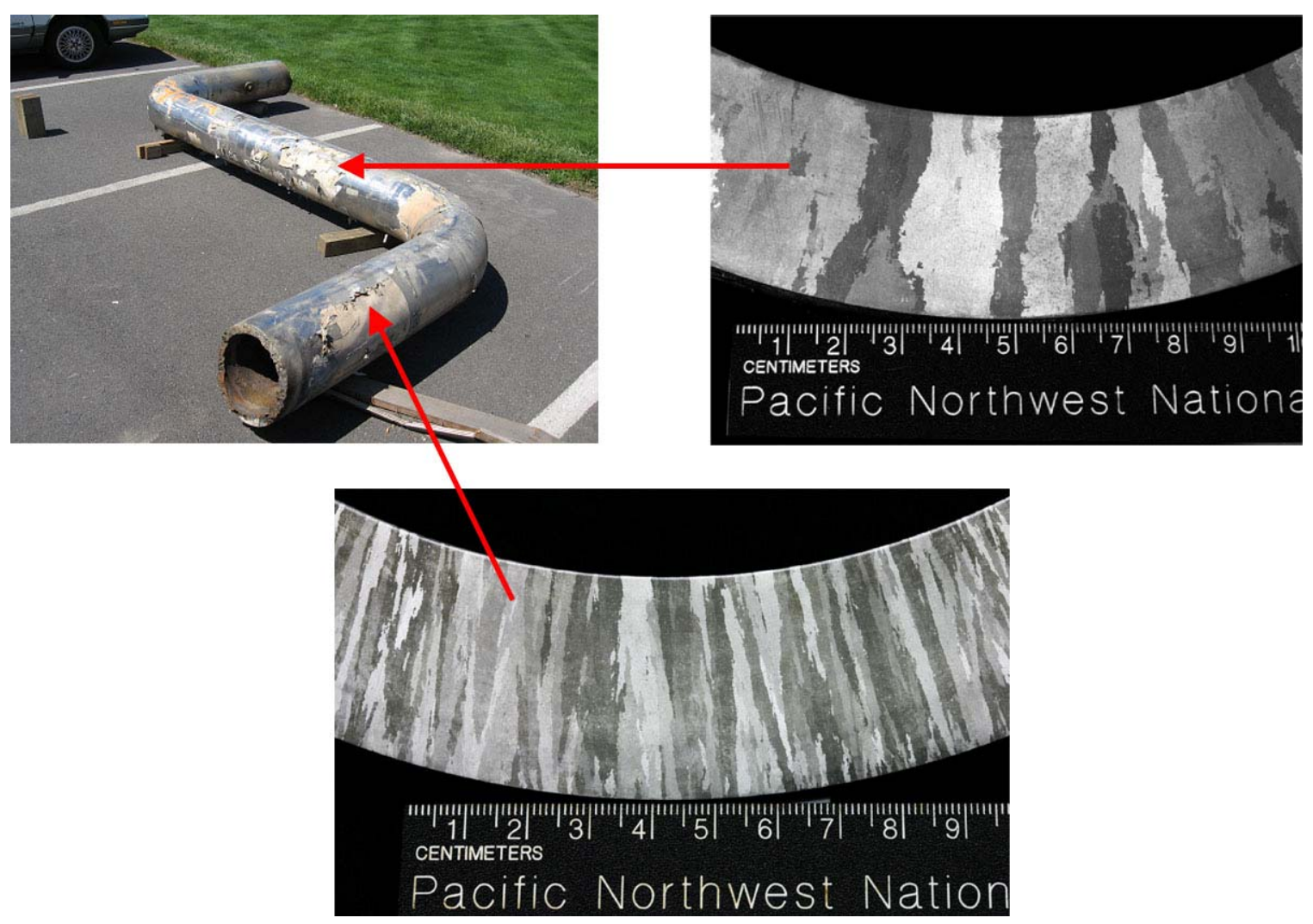

Figure 4.2. Photographs of Coarse-Grained Microstructures in a PZR Surge Line at PNNL 



\subsection{Phased Array Probes, Modeling, and Focal Law Development}

\subsection{Ultrasonic Probe Details}

The samples were examined using three phased array probes - a 1.5-MHz transmit-receive longitudinal (TRL) arrangement, an $800-\mathrm{kHz}$ TRL probe, and an $800-\mathrm{kHz}$ delta probe technique. The $1.5-\mathrm{MHz}$ transducer was chosen based on frequencies commonly used in reactor inspections of stainless steels. The $800-\mathrm{kHz}$ transducer was designed by PNNL to be able to detect flaws in 1-2-inch (25-50 $\mathrm{mm}$ ) thick CASS specimens based on previous PNNL experience in examining similar components. The delta technique was designed to detect crack tip signals in thicker ( $>2$ inch, or $>50 \mathrm{~mm}$ ) samples. The three models of transducers are described in Table 5.1.

Table 5.1. Ultrasonic Transducer Characteristics

\begin{tabular}{lccc}
\hline \multicolumn{1}{c}{ Probe } & $\begin{array}{c}\text { 1.5-MHz } \\
\text { TRL }\end{array}$ & $\begin{array}{c}\text { 800-kHz TRL } \\
\text { and Delta } \\
\text { Transmitter }\end{array}$ & $\begin{array}{c}\text { 800-kHz Delta } \\
\text { Receiver }\end{array}$ \\
\hline Active Aperture & $35 \mathrm{~mm}$ & $44 \mathrm{~mm}$ & $44 \mathrm{~mm}$ \\
Passive Aperture & $17.5 \mathrm{~mm}$ & $22 \mathrm{~mm}$ & $44 \mathrm{~mm}$ \\
Active Aperture Elements & 10 & 10 & 8 \\
Passive Aperture Elements & 3 & 5 & 8 \\
\hline
\end{tabular}

\subsection{Focal Law Development}

Before a phased-array probe can be used to perform an examination, a set of focal laws must be produced to control the firing of individual elements. The focal laws tell the Ultravision ${ }^{\circledR}$ control software which elements to fire at specific times to allow for proper beam-forming in the material to be examined. The focal laws also contain details about the angles being generated, the focal point of the sound, the delays associated with the wedge and electronics, and the orientation of the probe. PNNL uses a software package for producing focal laws named "ZETEC ${ }^{\circledR}$ Advanced Focal Law Calculator." The software package performs two functions: 1) focal law generation and 2) simulation of the ultrasonic field produced by the probe when using the generated laws. The user enters the physical information about the phased array probe, such as the number of elements and the sizes of the elements, and the wedge information, such as the wedge angle and the wedge size, into the program. The desired angles and focal distances are then entered, and the software generates the needed delays for each element to produce the desired beam steering and focusing in the material. The software beam simulation produces a simple image of the probe on the wedge, ray-tracing to show the focal depth and steering desired, and density mapping to enable the viewer to see how well the sound field responds for a particular angle and whether potential grating lobes exist that may be detrimental to the examination. Figure 5.1 shows an example of the ray-tracing for a probe on the left with the sound field density mapping on the right. It should be noted that this simulation is performed in isotropic material; i.e., the velocity of sound is 
maintained throughout any angle for a particular wave mode, which is not really the true state for CASS, but enables enhanced array design and focal law development.
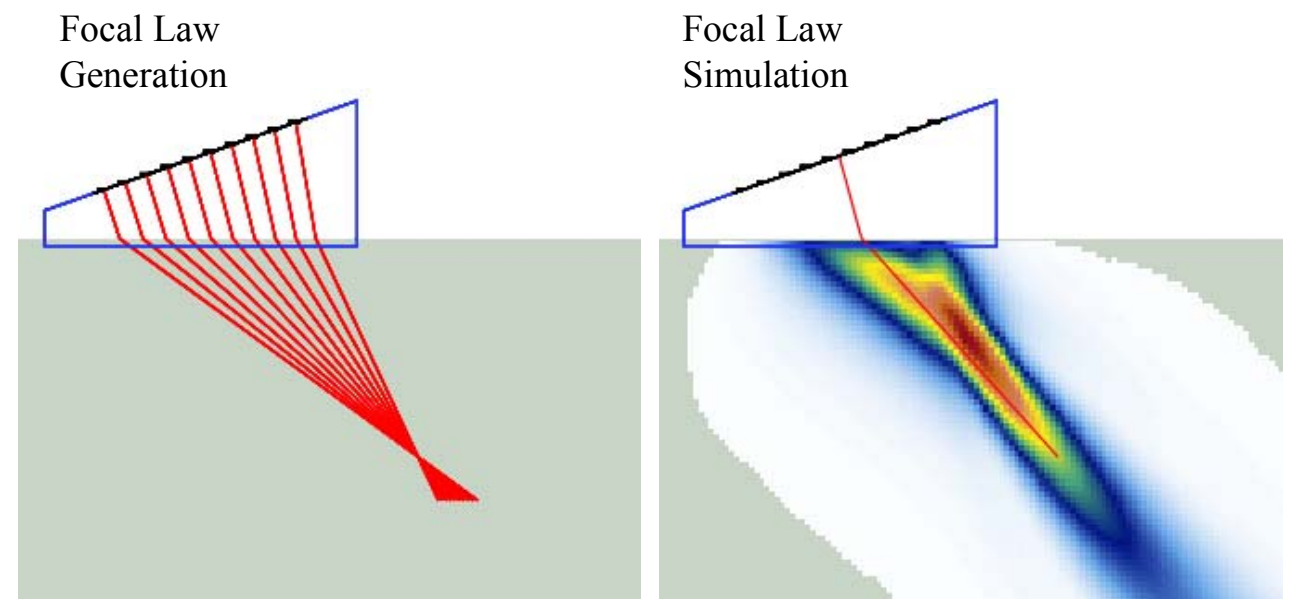

Figure 5.1. The ZETEC Advanced Phased Array Calculator is Useful for Generating Focal Laws (left) and Simulating the Sound Field for the Focal Law (right) to Determine Beam Characteristics

\subsection{5-MHz TRL Probe}

This 1.5-MHz TRL probe was originally designed by PNNL for examinations of wrought stainless steels, and consists of two 1.5-MHz phased array transducers mounted side-by-side on a Rexolite ${ }^{\circledR}$ wedge. The 1.5-MHz probes operated in the pitch-catch mode with wedges held together by an aluminum frame. The wedge dimensions were $49 \times 50 \mathrm{~mm}(1.93 \times 1.97 \mathrm{inch})$. Both transmit and receive probes were identical in design with a $3 \times 10$ element array, an active area of $35 \times 17.5 \mathrm{~mm}(1.38 \times 0.69 \mathrm{inch})$ and a greater than $60 \%$ bandwidth. The $1.5-\mathrm{MHz}$ probe has been used in previous examinations of flaws on the near and far sides of welds in wrought stainless steels. $1.5 \mathrm{MHz}$ is, however, generally considered to be too high in frequency for examining CASS components which have larger and more complex grain structures than wrought stainless steels. The 1.5-MHz TRL probe was included in this study because the probe has proven effective in examining materials of similar thickness to the surge line and provides a point of comparison for the other techniques. The probe is shown in Figure 5.2.

Specific focal laws were generated for the surge line based on the thickness of the piping and the width of the weld in the surge line pipe-to-elbow weld. After the focal laws were developed, the ultrasonic field that would be produced by these laws was simulated to demonstrate that the probe and the focal laws are able to insonify the region of interest. The simulations indicated that the $1.5-\mathrm{MHz}$ probe would be able to produce a well-focused beam at distances from directly below the probe to approximately $45 \mathrm{~mm}$ (1.8 inch) in front of the wedge. The probe produces a sound field that works best for detecting and sizing flaws on the near side of the weld, but it is also capable of detecting flaws from the far side of most welds in thinner materials, assuming that the microstructure allows the waves to form and propagate properly. Multiple focal laws were used in the examinations to obtain optimal results. Example simulations of the $1.5-\mathrm{MHz}$ probe steered to $30^{\circ}, 45^{\circ}$, and $60^{\circ}$, focused at a depth of $36 \mathrm{~mm}$ 


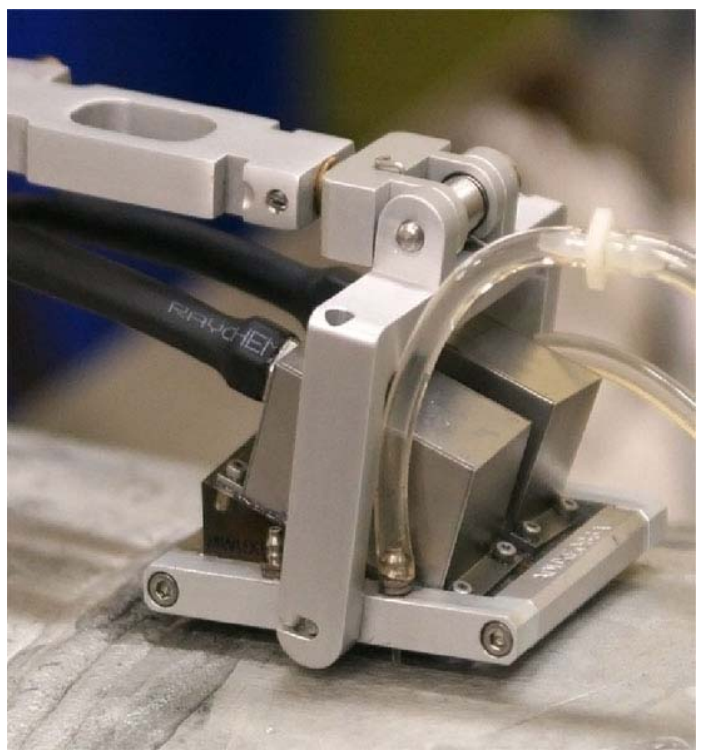

Figure 5.2. 1.5-MHz TRL Probe Originally Designed for Wrought Stainless Steel Welds

(1.4 inch), is shown in Figure 5.3. The wavelength for a 1.5-MHz probe is 0.15 inches $(3.8 \mathrm{~mm})$. The simulation shows the expected beam width to be 0.22 inches $(5.5 \mathrm{~mm})$ at $30^{\circ}, 0.24$ inches $(6.2 \mathrm{~mm})$ at $45^{\circ}$, and $0.39 \mathrm{inch}(9.8 \mathrm{~mm})$ at $60^{\circ}$.

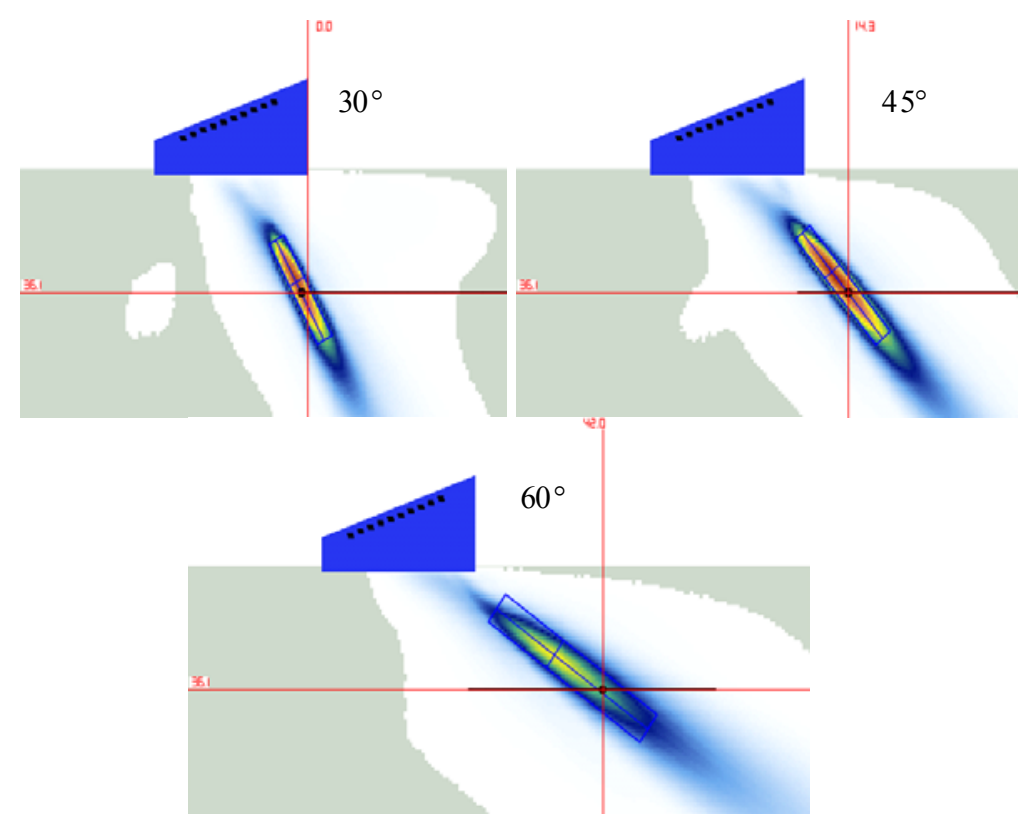

Figure 5.3. Simulations of the Beams Created by the $1.5-\mathrm{MHz}$ TRL Arrangement for $30^{\circ}, 45^{\circ}$, and $60^{\circ}$ 


\subsection{0-kHz 10×5 Probe}

The $800-\mathrm{kHz}$ probe was designed to provide a more optimal wavelength to allow the ultrasound to penetrate the large grain structure of CASS and maintain suitable resolution allowing the probe to resolve smaller flaws. The probes were mounted on a Rexolite ${ }^{\circledR}$ wedge side-by-side to operate in a pitch-catch mode and are shown in Figure 5.4. The wedges were separated by cork and held together with an aluminum frame. The wedge dimensions were $63 \times 63 \mathrm{~mm}(2.48 \times 2.48$ inches $)$. The transmit and receive probes were identical in design and consisted of a $10 \times 5$ element array with an active area of $43.2 \mathrm{~mm} \times 21.2 \mathrm{~mm}(1.70 \times 0.84 \mathrm{inch})$ and a greater than or equal to $50 \%$ bandwidth. The probes were designed at PNNL for this inspection and built by Imasonic ${ }^{\circledR}$. The wedges were curved to match the approximate 318 - $\mathrm{mm}$ (12.5-inch) diameter of the surge line piece.

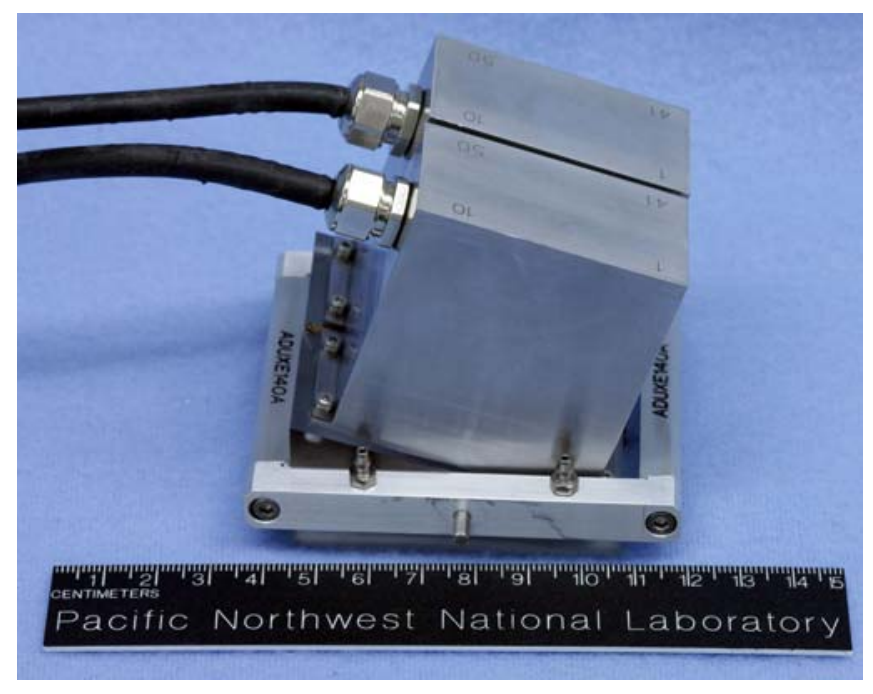

Figure 5.4. $800-\mathrm{kHz}$ TRL Probe

Specific focal laws were generated for the PZR surge line and WOG specimens based on the thickness of each specimen and the width of the welds in each specimen. Based on the focal laws and the results of the simulation, it was determined that the $800-\mathrm{kHz}$ probe was capable of effectively insonifying the region surrounding the weld in both samples. The $800-\mathrm{kHz}$ probe produces a 0.28 -inch $(7-\mathrm{mm})$ wavelength in stainless steels, almost twice the wavelength of the $1.5-\mathrm{MHz}$ probe. Example simulations of the $800-\mathrm{kHz}$ beam using one set of focal laws are shown in Figure 5.5.

The $800-\mathrm{kHz}$ probe was found to produce a beam with a width of $7 \mathrm{~mm}(0.28$ inch $)$ at $30^{\circ}, 9 \mathrm{~mm}$ ( $0.35 \mathrm{inch})$ at $45^{\circ}$, and $13 \mathrm{~mm}(0.51 \mathrm{inch})$ at $60^{\circ}$. The $800-\mathrm{kHz}$ probe is able to provide an effective sound field at distances from directly under the probe to a distance of $36 \mathrm{~mm}$ (1.42 inch) in front of the wedge. As with the 1.5-MHz probe, the ability of the probe to accurately measure crack width and depth improves the closer to the probe focal spot the flaw is located as this is the location of tightest focusing. Because the $800-\mathrm{kHz}$ beam focal zone does not extend as far out in front of the probe as the $1.5 \mathrm{-MHz}$ beam, it needs to be positioned closer to the flaw than the $1.5-\mathrm{MHz}$ probe. 


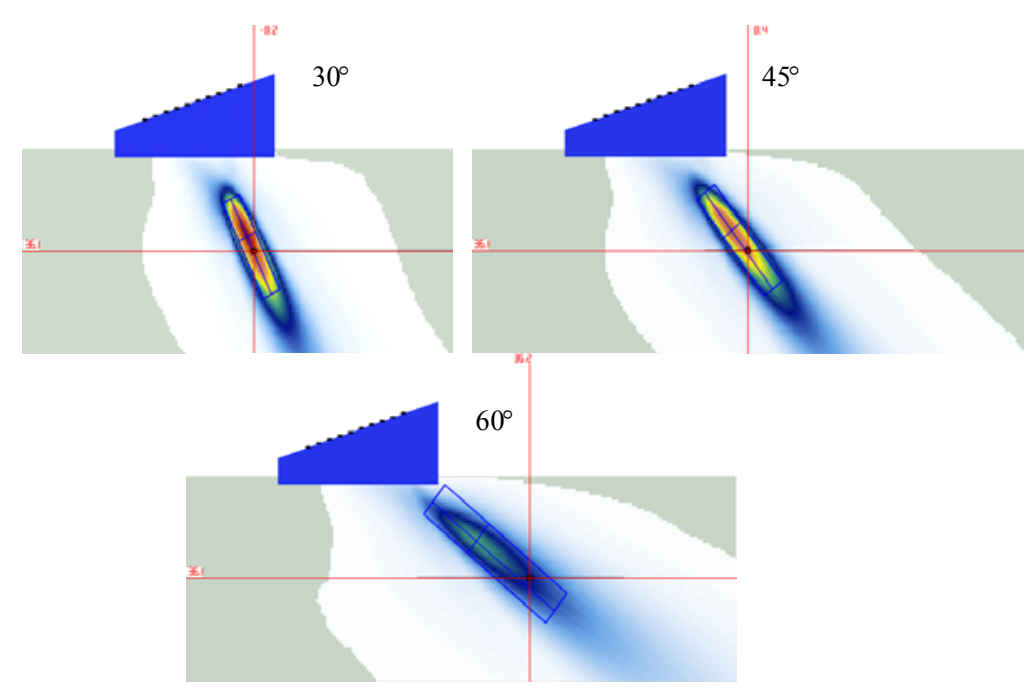

Figure 5.5. Simulations of the Beams Created by the $800-\mathrm{kHz}$ TRL Arrangement for $30^{\circ}, 45^{\circ}$, and $60^{\circ}$

\subsection{0-kHz Delta Probe}

The delta probe used an $800 \mathrm{kHz}, 10 \times 5$ element probe and an $800 \mathrm{kHz} 8 \times 8$ element receiver mounted on wedges and held face-to-face in a variable-displacement holder. The delta probe is significantly more complex than the other probes as optimum spacing between probes and position relative to a flaw need to be determined. The transducers and holder are shown in Figure 5.6.

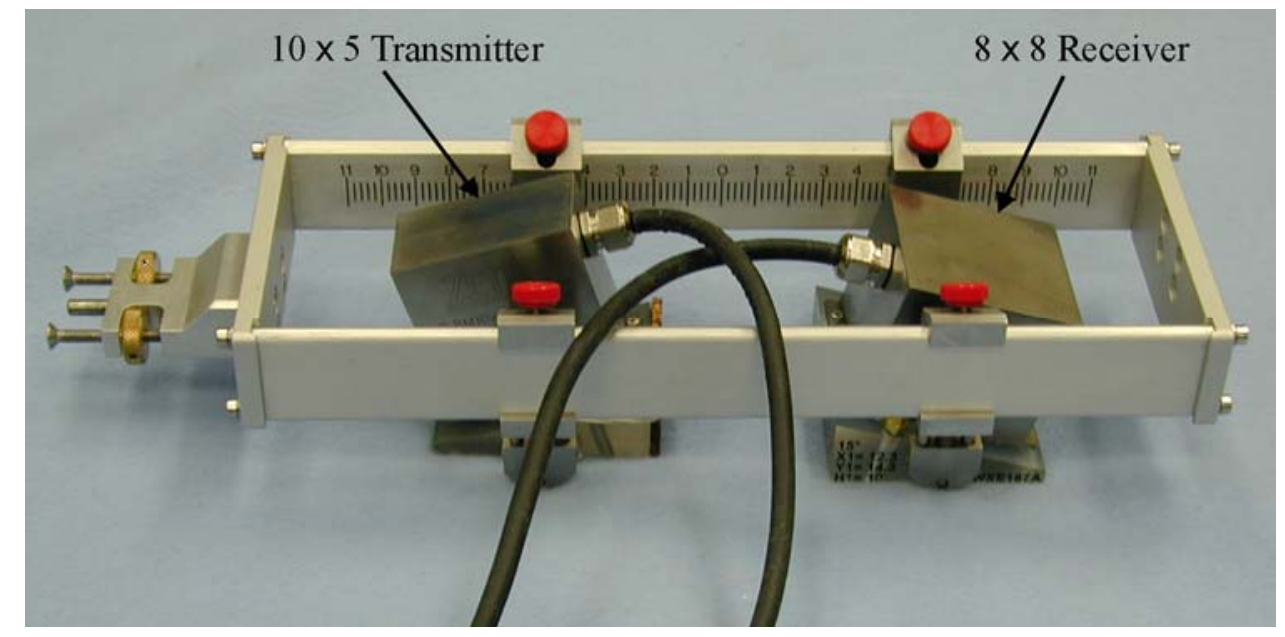

Figure 5.6. Delta Probe Arrangement

Focal law generation was complex for the delta probe. The ZETEC ${ }^{\circledR}$ software used to generate the standard focal laws for the other two probes is not able to generate the focal laws for this arrangement, as the software is not designed to handle the specialized delta technique. To perform the delta technique, a series of focal laws were developed by generating laws individually for the transmitter and receiver, and 
then manually assembling a composite focal law using spreadsheets and text editors. The laws were tested using test blocks to determine that they were accurate.

The delta technique probe was used in two modes, depending on the thickness of the sample. It was seen that the transmitter was not able to project an effective beam in the 1-inch (25.4-mm) WSS coupons or the 1.25-inch (31.8-mm)-thick PZR surge line; the simulations showed that the $800-\mathrm{kHz}$ transmitter probe was not able to project a good sound field underneath the receiver as well. In the 2-3-inch (50.8$76.2 \mathrm{~mm}$ )-thick WOG specimens, the receiver was able to project a good sound field under the receiver. For the thicker specimens a set of laws using the typical delta probe method was used. The transmitter was aimed at the center of the sample at a fixed angle to insonify the flaw. The receiver was used to listen at a variety of depths to determine the location of any possible tip diffraction signal. After experimentation it was determined that the best angle for transmittal was $55^{\circ}$. The simulated sound field for the $10 \times 5$ transmitter set to $55^{\circ}$ is shown in Figure 5.7 and the example simulations of the sound field for the receiver focused under the probe is shown in Figure 5.8. The transmitter is able to focus fairly well at a distance of $1 \mathrm{inch}(25 \mathrm{~mm})$ in front of the wedge, and the receiver can focus well at 0.5 inches $(12.5 \mathrm{~mm})$ under the probe.

For the thinner samples, the focal laws were developed to allow the receiver to listen 0.5 inch $(12 \mathrm{~mm})$ in front of the wedge. The focal laws for the thinner samples somewhat resemble a phased array time-of-flight-diffraction (TOFD) setup more than a delta probe setup, but it showed better sensitivity to the flaws in the thinner samples than attempts at a more conventional delta probe setup. Different sets of focal laws were made for each sample examined. Example simulations of one set of focal laws for the transmitter are shown in Figure 5.9, and a sample set of simulations of focal laws for the receiver are shown in Figure 5.10.

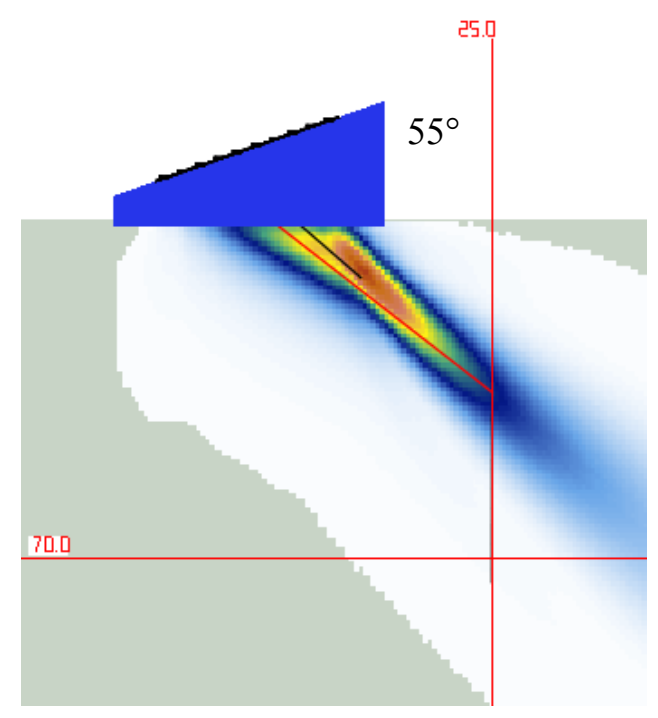

Figure 5.7. Simulated Sound Field for the $10 \times 5$ Transmitter at $55^{\circ}$ for Delta-Technique Evaluations 

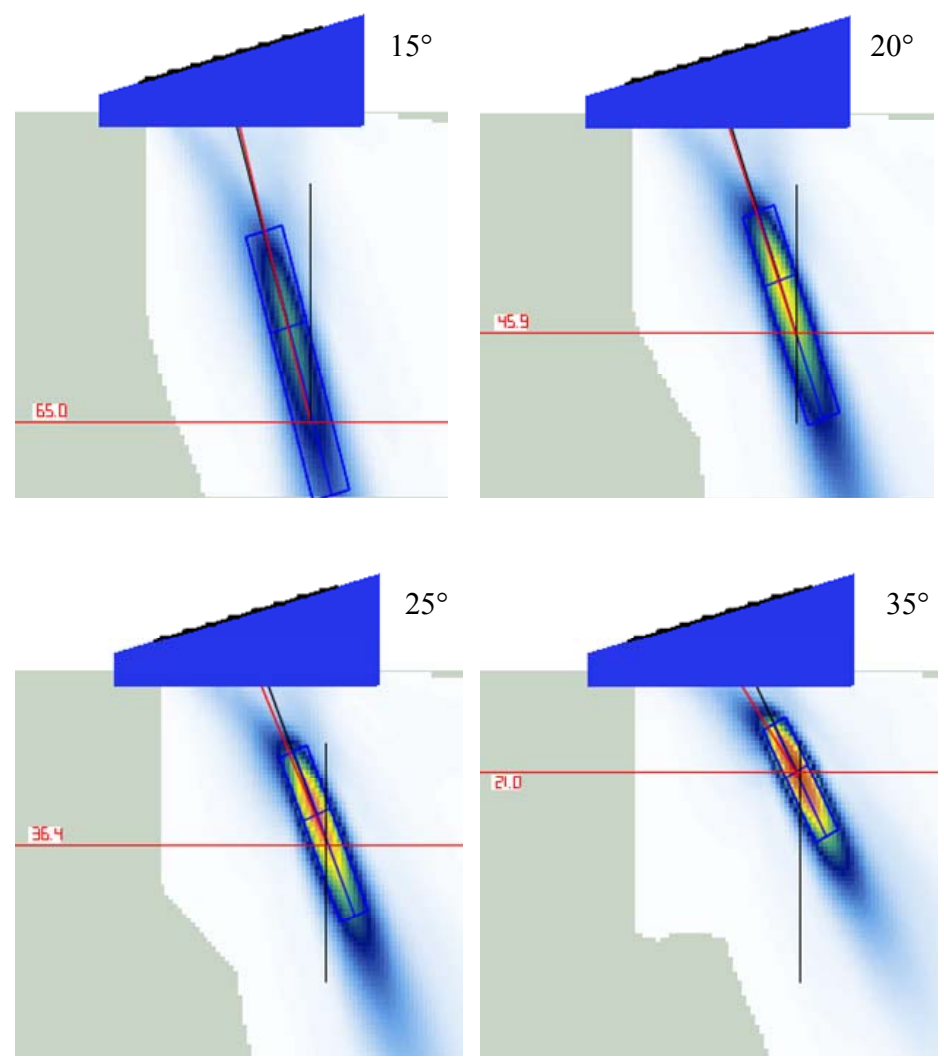

Figure 5.8. Simulated Sound Fields for the Receiver Focused 0.5 Inches under the Front of the Wedge

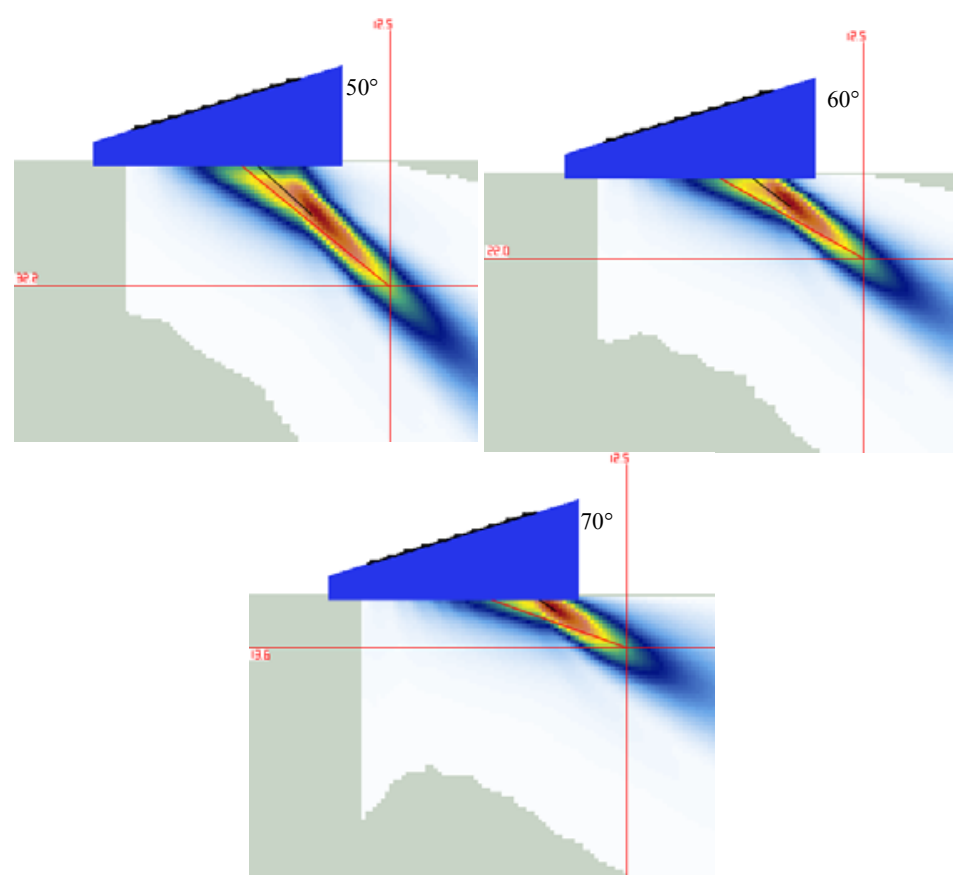

Figure 5.9. Simulated Sound Fields for the Transmitter Focused 0.5 Inch in Front of the Wedge 

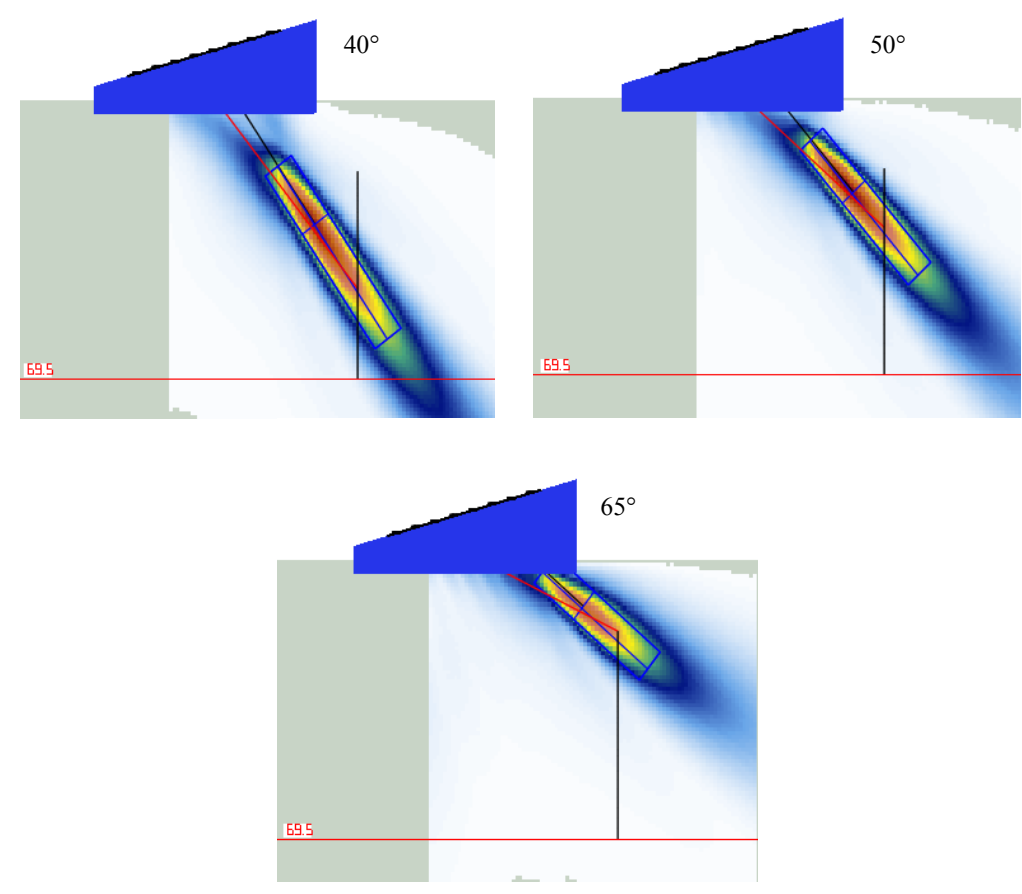

Figure 5.10. Simulated Sound Fields for the Receiver Focused 0.5 Inch in Front of the Wedge 


\subsection{Data Acquisition Set-Up and Configuration}

Conventional phased-array and delta-technique data were acquired on specimens in the 12-14 inch (305-356 mm) diameter range using a manual scanner while data acquired on the larger diameter pipe section (32 inch [813 mm]) were acquired with a flat track scanner. Each of these setups is described below.

\subsection{Phase Array Data Acquisition (PZR Surge Line and WOG Specimens)}

Set-up and laboratory configuration for $800-\mathrm{kHz}$ and $1.5-\mathrm{MHz}$ data acquisition on the PZR surge line required the use of the manual-encoded scanner mounted on an adjustable guide track or ring supported by a custom-designed floor-to-ceiling brace. The setup is shown in Figure 6.1 with the manual scanner on the guide ring section. The probe is mounted on an extension arm which is adjustable along the pipe axis. Two encoders on the manual track scanner provide positional information in either or both the axial and circumferential directions for line and raster scanning
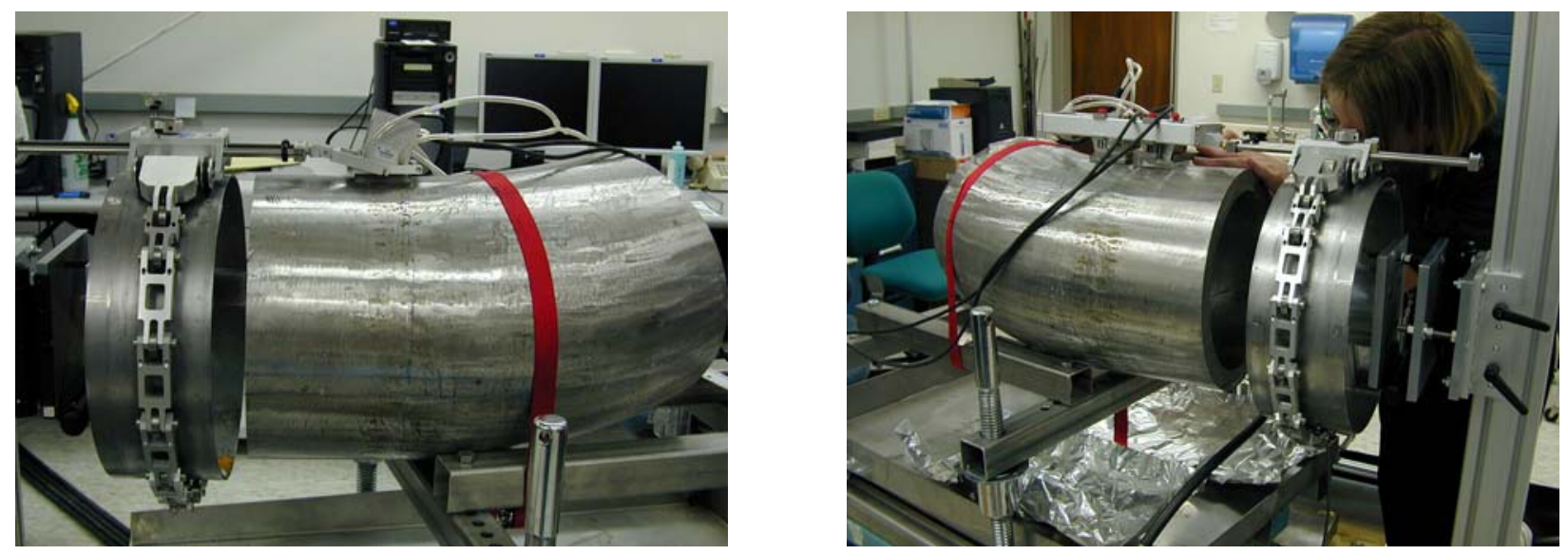

Figure 6.1. Photographs of the PZR Surge Line Specimen. Left: Configured for standard PA-UT with the pipe-end on the left and the elbow-end on the right side of the picture. Right: The guide ring and floor-to-ceiling brace are shown to the right.

In order to establish adequate acoustic coupling between the probe wedge and the specimen surface, a flow loop was designed to pump water through nipple fixtures embedded on both sides of the wedge harness that direct the flow evenly over the surface of the specimen to be imaged. As the water couplant flowed off of the top of the specimen, it was collected in a tray, channeled back to the pump, and recycled. For more effective translation of the probe over the specimen surface, small amounts of dish soap (surfactant) were added to the water-couplant bath, improving coupling efficiency and transducer movement.

Data acquisition was accomplished using the low-frequency DYNARAY ${ }^{\circledR}$ system for the $800-\mathrm{kHz}$ probe. This beta system is equipped to accommodate a maximum of 128 channels from phased array 
probes and runs Ultravision ${ }^{\circledR} 3.0 \mathrm{~B} 1$ software. Its low-frequency pulsing will drive probes in the $0.2-20 \mathrm{MHz}$ range. The 1.5-MHz data were acquired with the standard Tomoscan ${ }^{\circledR}$ III system, which has 32 channels in transmission and up to 64 channels in the receive mode. It runs Ultravision ${ }^{\circledR} 1.1 \mathrm{Q} 5$ software and is designed to drive probes in the $1-25 \mathrm{MHz}$ range.

The setup for scanning the WOG specimens with the $800-\mathrm{kHz}$ probe is shown in Figure 6.2. A specimen is immersed in water and a flat track peg scanner is used for positioning and moving the probe. The scanner is controlled by PNNL's SAFT system electronics while the ultrasonic data is acquired with the DYNARAY ${ }^{\circledR}$ system. Both line and raster scans were acquired with positional information recorded. The probe's Rexolite ${ }^{\circledR}$ wedge was machined to conform to the 32-inch OD of the pipe segments.
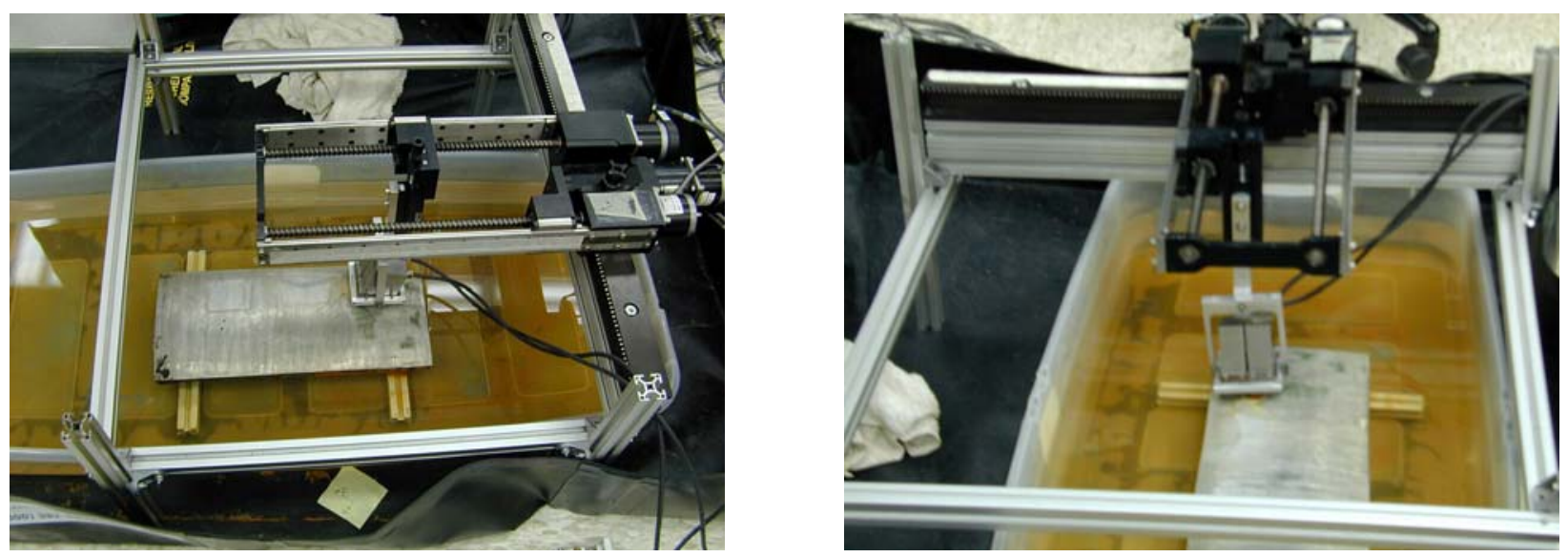

Figure 6.2. Top and Front Views of the Immersion Tub Scanning Configuration for the PNNL CASS Specimen Using an Automated X-Y Axis, Flat Peg-Scanner and Spring-Loaded Gimbal Apparatus

\subsection{0-kHz Delta-Technique Data Acquisition}

The delta technique setup includes configuring two $800-\mathrm{kHz}$ phased array probes in an arrangement similar to that of a TOFD method, where transmit and receive transducers are oriented opposite and facing each other, with some separation distance. This system required the use of one of the previously used $800-\mathrm{kHz}$ transducers (with $5 \times 10$ elements) to serve as the transmit transducer and the new $800-\mathrm{kHz}$ probe (with $8 \times 8$ elements) that acted as the receive transducer. Both transducers were machined to conform to the surge line diameter, and mounted into a custom designed chassis shown earlier in Figure 5.5 and in Figure 6.3 on the notched specimen. 


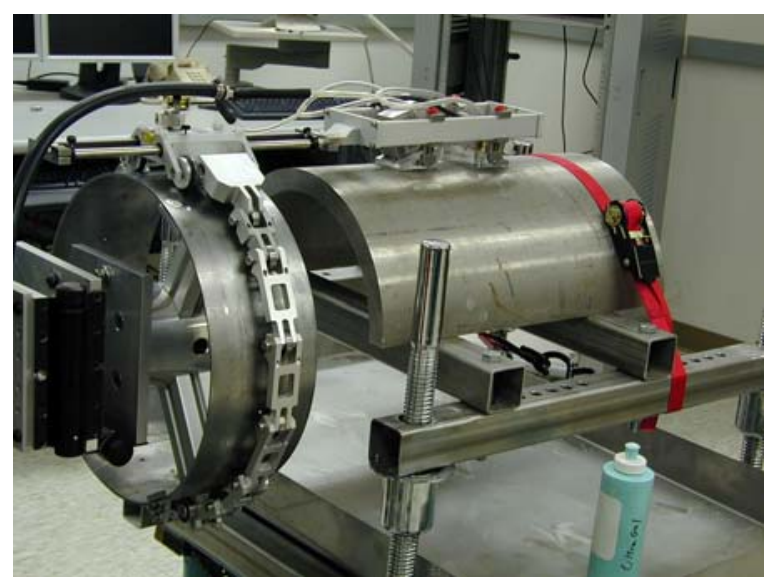

Figure 6.3. Photograph of Delta-Technique Configuration Just Prior to Scanning

With the water-couplant flow-recycle system turned on, the ultrasonic signals are viewed and analyzed in real-time and positional, separation, and alignment adjustments are made. Several focal law files were created and tested, and it was determined that a focal law using a range of angles from $30^{\circ}-75^{\circ}$ focused at a focal plane of 0.47 inch $(12 \mathrm{~mm})$ into the part was ideal for the WSS coupons. With this law file, a physical probe separation of $0.8 \mathrm{inch}(20.3 \mathrm{~mm})$ yielded the best resolution and imaging capabilities. It was also noted that when scanning over the $20 \%$ and $40 \%$ EDM notches, the probes are best positioned at a separation distance that is equidistant from each other with the EDM notch in the center, between the transducers.

Once the system was properly tuned and the encoders calibrated, data collection was initiated on each of the EDM notched coupons on both pipe segments containing notches of $10 \%, 20 \%, 30 \%$, and $40 \%$ through-wall. Line scans were made that encompassed the entire notch since the scan was set up in the parallel direction to the notch as well as extra pipe material on each side of the notch for comparison. Upon completion of the EDM notch scans, similar setup and scans were completed on the PZR surge line specimen in the flaw regions using the same procedures.

Finally, raster scans were conducted on the WOG and PNNL specimens using the delta technique with some minor setup modifications. These scans required the use of the peg scanner with the WOG specimens and the PNNL CASS specimen submersed in the water tub as described earlier. Since the sections of the WOG and PNNL specimens analyzed were submerged in a water bath, the mismatch of the delta wedge contours verses the OD of the pipe segments were minimized because the water filled in the gaps creating sufficient acoustic coupling. Figure 6.4 illustrates top and side views of the deltatechnique evaluation on the PNNL CASS specimen in the plastic immersion tank. 

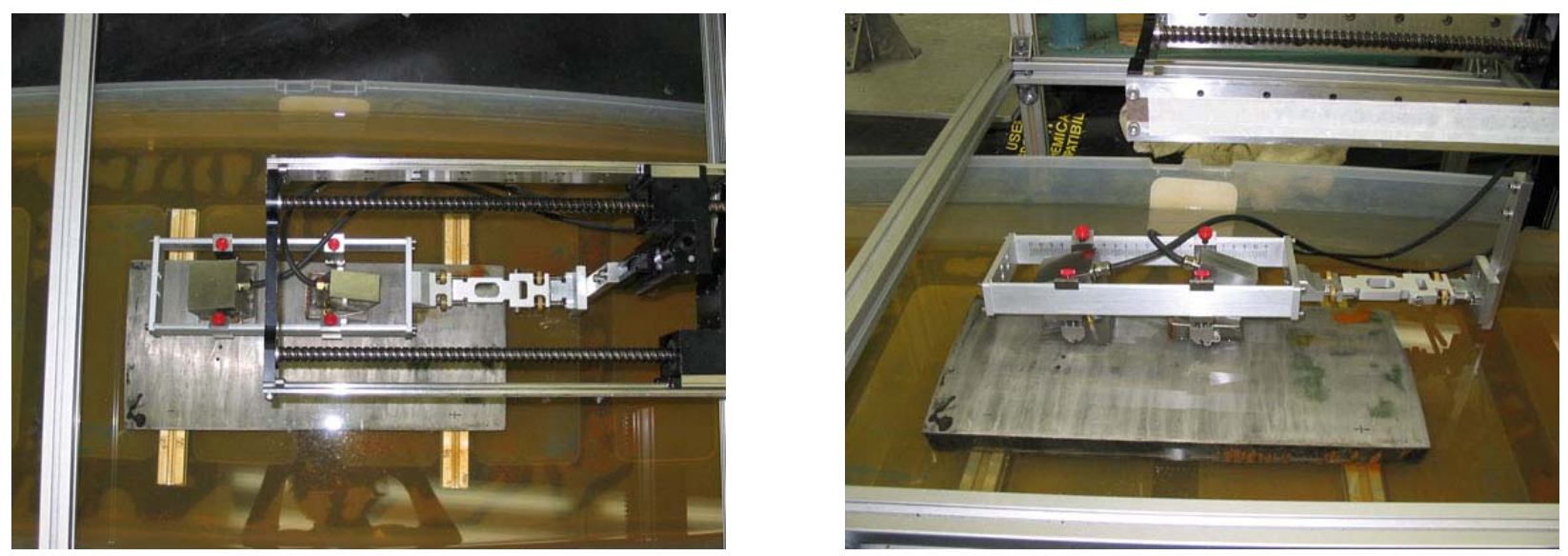

Figure 6.4. Top and Side Views of the Delta-Technique Chassis Mounted on a PNNL CASS Specimen for Data Acquisition, Using the Automated Peg-Scanner in an Immersion Tub 


\subsection{Specimens Evaluated in this Study}

\subsection{CASS PZR Surge-Line Specimen}

The surge-line segment is 12 -inch-(304.8-mm) diameter Schedule 160 pipe and is comprised of ASME SA-351 GR CF-8M stainless steel. This pipe segment is approximately 18 inches $(457.2 \mathrm{~mm})$ in length, has a 12.75 -inch $(323.9 \mathrm{~mm}$ ) outside diameter (pipe side), and an approximately 1.25 -inch (31.8-mm) wall thickness on the centrifugally cast side of the weld. The CASS PZR surge-line weld specimen was salvaged from a cancelled plant and four thermal fatigue cracks were implanted into the weld region. The technique includes excavation of parent/weld material in the area targeted for implantation, and then subsequent insertion of the coupon containing the thermally induced fatigue crack (see Figure 7.1). The flaw implantation vendor (FlawTech ${ }^{\circledR}$, Inc.) employs a unique proprietary process where heating/cooling under tension is used to initiate a thermal fatigue crack in a coupon. The number of cycles is controlled to obtain the desired crack depth and "roughness." After taking exact physical measurements, the flaw implant is seal welded in place to establish final location and size. The remaining weld groove is then filled with a standard welding procedure. The disadvantage is that the coupon is surrounded by weld metal that may potentially introduce additional UT reflectors; however, for studies on coarse-grained CASS materials, it was assumed that any reflectors left over from the implantation process would not be detectable because of the noise level inherent in the cast microstructures of this specimen. This indeed was the case in this study.
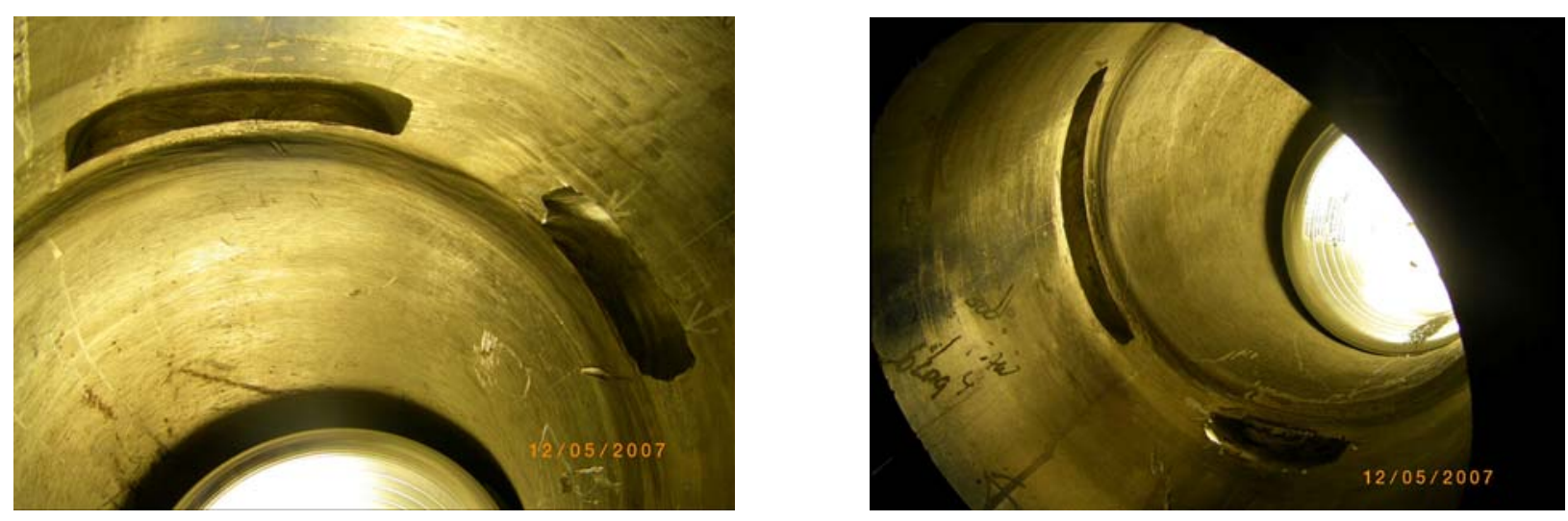

Figure 7.1. Photographs of the Inside Surface of the CASS PZR Surge-Line After Excavation and Preparation of the Areas Targeted for Flaw Implantation (courtesy of FlawTech ${ }^{\circledR}$, Inc.)

Four implanted thermal fatigue cracks were introduced into this specimen with the following characteristics:

$\begin{array}{lll}\text { - Flaw \#1: } & \text { Orientation: } & \text { Circumferential } \\ & \text { Type: } & \text { Thermal fatigue (coupon implant) } \\ & \text { Length: } & 2 \text { inches }(50.8 \mathrm{~mm}) \\ \text { Depth: } & 30 \% \text { through-wall } \\ & \text { Position: } & \text { in the weld (along centerline of weld) }\end{array}$


- Flaw \#2: Orientation: Circumferential

Type: $\quad$ Thermal fatigue (coupon implant)

Length: $\quad 2$ inches $(50.8 \mathrm{~mm})$

Depth: $\quad 30 \%$ through-wall

Position: $\quad$ in parent material - centrifugally cast pipe side (near weld fusion line)

- Flaw \#3: Orientation: Circumferential

Type: $\quad$ Thermal fatigue (coupon implant)

Length: $\quad 4$ inches $101.6 \mathrm{~mm}$ )

Depth: $\quad 35 \%$ through-wall

Position: $\quad$ in the weld (along centerline of weld)

- Flaw \#4: Orientation: Circumferential

Type: $\quad$ Thermal fatigue (coupon implant)

Length: $\quad 6$ inches $152.4 \mathrm{~mm})$

Depth: $\quad 30 \%-50 \%$ through-wall (variable depth), with $50 \%$ as the deepest point

Position: $\quad$ in the weld (along centerline of weld)

A summary of flaw position, location, orientation, length, and depth (flaw height) is shown in Table 7.1.

Table 7.1. Positional and Dimensional Data for Implanted Flaws in the CASS PZR Surge Line

\begin{tabular}{|c|c|c|c|c|c|c|}
\hline Flaw & Flaw Type & Flaw Location & $\begin{array}{c}\text { Flaw } \\
\text { Orientation }\end{array}$ & Flaw Length & $\begin{array}{c}\text { Flaw Depth } \\
\text { (Height) }\end{array}$ & $\begin{array}{c}\text { Degree } \\
\text { Location }\end{array}$ \\
\hline 1 & Thermal Fatigue & Weld Center Line & Circumferential & $\begin{array}{c}4.0 \text { in. } \\
(10.2 \mathrm{~cm})\end{array}$ & $35 \% \mathrm{~T}$ & $45^{\circ}$ \\
\hline 2 & Thermal Fatigue & $\begin{array}{l}\text { Pipe Side Near } \\
\text { Fusion Line }\end{array}$ & Circumferential & $\begin{array}{l}2.0 \mathrm{in} . \\
(5.1 \mathrm{~cm})\end{array}$ & $30 \% \mathrm{~T}$ & $120^{\circ}$ \\
\hline 3 & Thermal Fatigue & Weld Center Line & Circumferential & $\begin{array}{l}2.0 \mathrm{in} . \\
(5.1 \mathrm{~cm})\end{array}$ & $30 \% \mathrm{~T}$ & $210^{\circ}$ \\
\hline 4 & Thermal Fatigue & Weld Center Line & Circumferential & $\begin{array}{c}6.0 \text { in. } \\
(15.2 \mathrm{~cm})\end{array}$ & $30 \%-50 \% \mathrm{~T}$ & $300^{\circ}$ \\
\hline
\end{tabular}

\subsection{WOG Specimens}

Three welded piping specimens fabricated by industry's Westinghouse Owners Group, on loan to PNNL from the Electric Power Research Institute (EPRI), were used for this study. These specimens represent typical configurations of several components in the primary coolant loop of Westinghousedesigned plants, and surface-breaking thermal or mechanical fatigue cracks located on either side of a weld. The WOG (traveling set of component segments) specimens represent a variety of reactor piping configurations, including

- SCSS elbow to centrifugally cast pipe

- carbon steel inlet nozzle to forged stainless steel safe-end to statically cast elbow

- SCSS pump outlet nozzle to CCSS pipe 
- clad carbon steel outlet nozzle to forged stainless steel safe-end to centrifugally cast pipe.

Represented microstructures are described as coarse and coarse-mixed microstructures, consisting of combined dendritic (columnar) and equiaxed grains. The flaws in these specimens are basically considered to be planar cracks oriented parallel to the weld centerline, and perpendicular to and connected to the inside diameter surface. However, the fabrication processes have also created some transverse cracking in some of the specimens. In general, the tightness and branched orientations of the thermal fatigue cracks make them more difficult to ultrasonically detect in comparison to the mechanical fatigue cracks. Each specimen has been assigned a designation that indicates the mock-up configuration. The piping configurations and crack details for the WOG specimens used in this study are described in Table 7.2. These estimated depth values were provided by EPRI. Photographs showing the WOG specimens are presented in Figure 7.2. Note the varied inside and outside diameter surface conditions indicative of piping geometries installed in the field.

Table 7.2. WOG Specimens Used in This Study

\begin{tabular}{llccl}
\hline $\begin{array}{c}\text { WOG } \\
\text { Specimen }\end{array}$ & Mock-up Configuration & $\begin{array}{c}\text { Crack } \\
\text { Type }\end{array}$ & $\begin{array}{c}\text { Crack Through- } \\
\text { Wall (\%) }\end{array}$ & $\begin{array}{c}\text { Crack Location } \\
\text { (Side of Weld) }\end{array}$ \\
\hline MPE-6 & CCSS pipe-to-SCSS elbow & TF & 18 & SCSS (elbow) \\
ONP-3-5 & CCSS pipe-to-WSS safe end & TF & 28 & WSS (safe-end) \\
OPE-5 & CCSS pipe-to-SCSS elbow & TF & 23 & SCSS (elbow) \\
\hline
\end{tabular}

Specimen MPE-6 is 26.0-cm (10.25-inch) wide (circumferential distance) and 60.96-cm (24-inch) long in axial extent. This specimen is a statically cast elbow to centrifugally cast pipe section, where the elbow side is $8.38-\mathrm{cm}$ (3.3-inch) thick and the pipe side is $6.6-\mathrm{cm}$ (2.6-inch) thick. Both the statically cast and centrifugally cast microstructures are defined as a coarse-mixed matrix of grains (see the micrograph in Figure A.34 for a visual depiction of the microstructure for this specimen). The crack in MPE-6 is a thermal fatigue crack on the elbow side of the weld centerline, with a circumferential extent (length) of $5.92 \mathrm{~cm}(2.33 \mathrm{inch})$ and a depth of $1.50 \mathrm{~cm}(0.59 \mathrm{inch})$.

Specimen ONP-3-5 is 20.3-cm (8.0-inch) wide (circumferential distance) and 61.6-cm (24.25-inch) long in axial extent. This specimen is a clad carbon steel outlet nozzle to forged stainless steel safe end to centrifugally cast pipe section, where the nozzle side consists of $6.60-\mathrm{cm}(2.6$-inch) thick carbon steel and $0.51-\mathrm{cm}(0.2$-inch) thick clad. The forged stainless steel safe end is $7.11-\mathrm{cm}(2.8$-inch) thick, and the CCSS pipe segment is 6.35 -cm (2.5-inch) thick. Both clad carbon steel and forged stainless steel segments are defined as consisting of a fine-grained microstructure, while the centrifugally cast microstructure is defined as a coarse matrix of grains. The crack in ONP-3-5 is a thermal fatigue crack on the safe-end side of the weld centerline between the nozzle segment and the forged stainless steel safe-end segment, with a circumferential extent (length) of $6.6 \mathrm{~cm}$ ( $2.6 \mathrm{inch})$ and a depth of $1.78 \mathrm{~cm}(0.7 \mathrm{inch})$. 

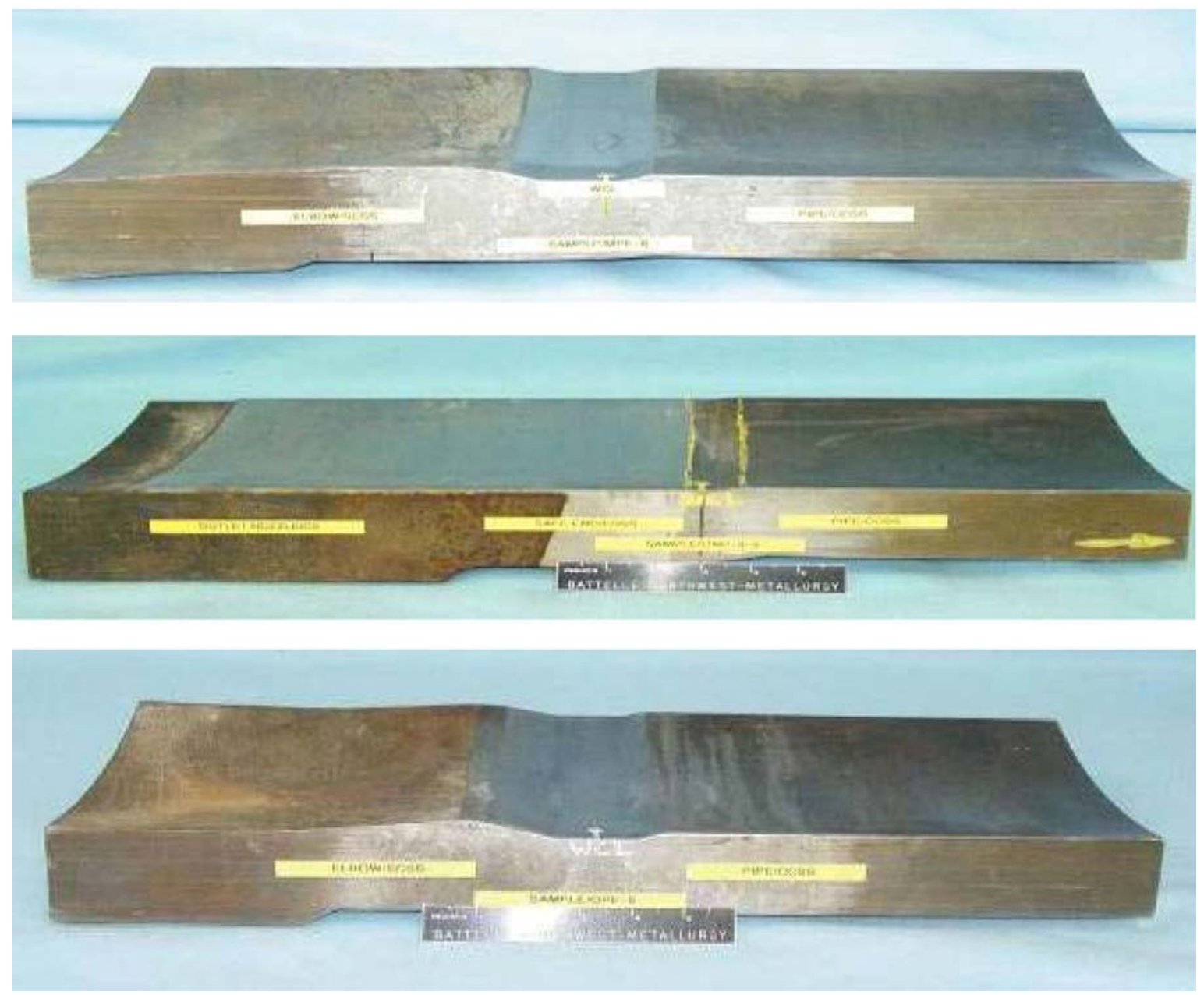

Figure 7.2. Photographs of the Three WOG Specimens Examined - (top): WOG specimen MPE-6; (center): WOG specimen ONP-3-5; (bottom): WOG specimen OPE-5

Specimen OPE-5 is 20.3-cm (8.0-inch) wide (circumferential distance) and 52.1-cm (20.5-inch) long in axial extent. This specimen is a statically cast elbow to centrifugally cast pipe section, where the elbow side is $7.11-\mathrm{cm}(2.8$-inch) thick and the pipe side is $5.84-\mathrm{cm}(2.3$-inch) thick. Both the statically cast and centrifugally cast microstructures are defined as a coarse matrix of grains. The crack in OPE-5 is a thermal fatigue crack on the elbow side of the weld centerline, with a circumferential extent (length) of $6.15 \mathrm{~cm}(2.42 \mathrm{inch})$ and a depth of $1.63 \mathrm{~cm}(0.64 \mathrm{inch})$.

\subsection{Wrought Steel Coupons with EDM Notches}

Two wrought stainless steel coupons were cut from a larger section of wrought piping available here at PNNL. Figure 7.3 provides a photograph of the parent piping material from which these coupons were extracted. 


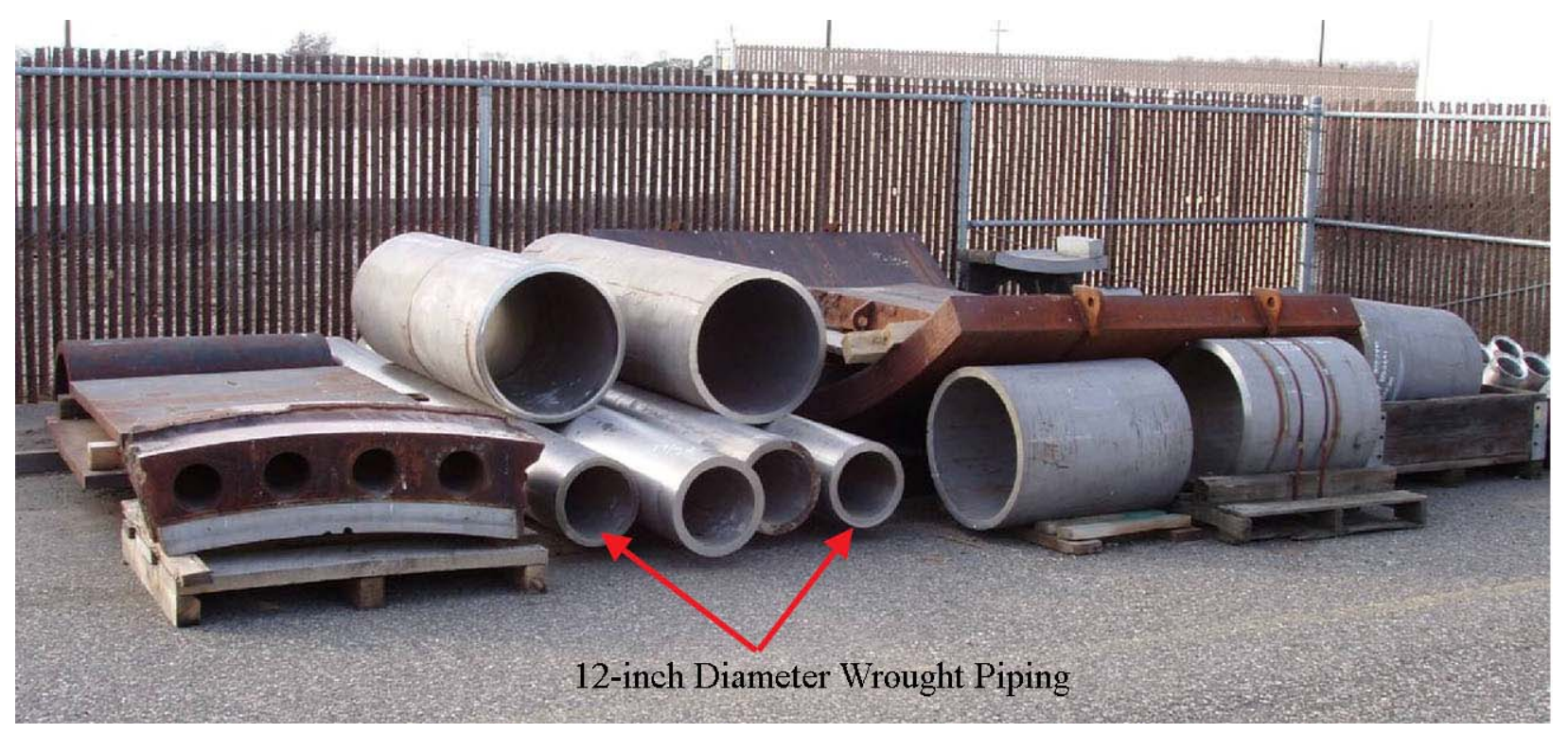

Figure 7.3. Photographs of the WSS Parent Material from which the Two Calibration Coupons (with EDM notches) were Extracted

The coupons are 18 -inch $(45.7-\mathrm{cm})$ long, and are $180^{\circ}$ circumferential sections with a 1-inch $(2.54-\mathrm{cm})$ wall thickness. Two EDM notches were introduced into the center of each of the coupons approximately 9 inch $(22.9 \mathrm{~cm})$ from either end and equally offset from the $0^{\circ}$ reference point on each coupon. The EDM notches are 2.5 -inch $(6.35-\mathrm{cm})$ long as measured on the ID surface of the coupons and are $10 \%, 20 \%, 30 \%$, and $40 \%$ through-wall in depth, corresponding to 0.1 -inch $(2.54-\mathrm{mm}), 0.2$-inch (5.08-mm), 0.3-inch $(7.62-\mathrm{mm})$, and 0.4-inch $(10.16-\mathrm{mm})$ deep, respectively. Figure 7.4 provides photographs of one of the EDM notch WSS coupons used for the PA delta-technique evaluation for detection of tip-diffracted energy in CASS.
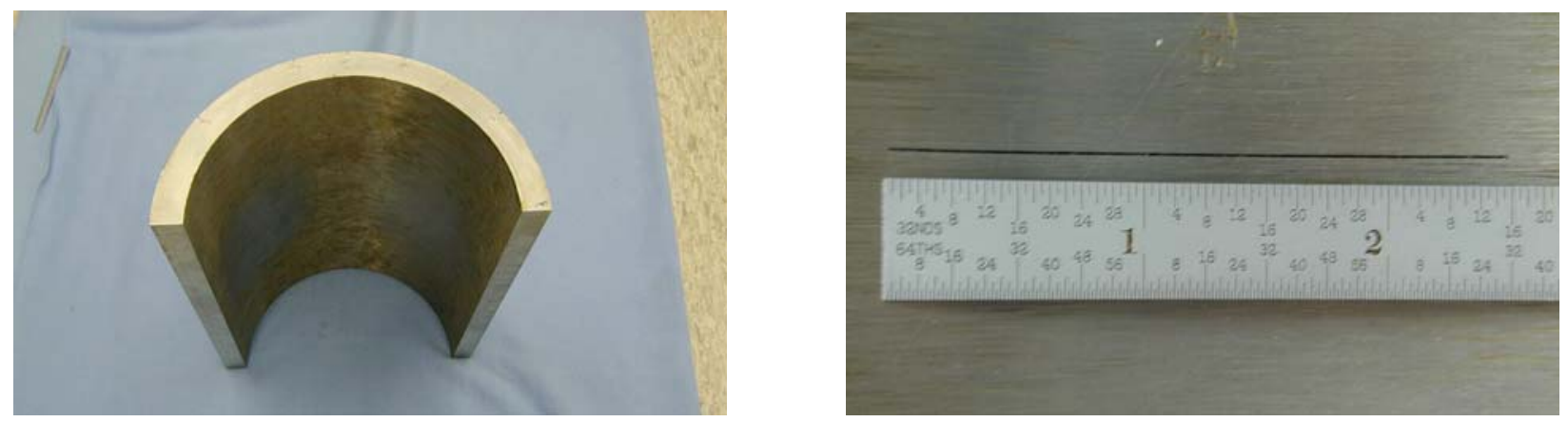

Figure 7.4. Photographs of the End View and ID Surface View for One of the WSS Coupons with EDM Notches, Used for Evaluation and Baseline Measurements of the PA Delta-Technique 


\subsection{PNNL CASS Specimen}

The PNNL CASS specimen consisted of a section cut from butt-welded, 845-mm (33.3-inch)-OD, 60-mm (2.4-inch)-thick CCSS pipe. This pipe material was from two different heats of ASTM A-351 Grade CF-8A, a cast 304 material (Diaz et al. 1998). This specimen has been used in a variety of other studies, and has label and identification numbers scribed on it; these labels/scribe numbers are used to aid in distinguishing individual specimens from each other. This specimen contains a weld made by personnel qualified to meet Section III requirements of the ASME Code; the weld was made under shop conditions but is typical of field practice. The weld crown was ground relatively smooth and blended with the parent pipe, although troughs between weld paths are still present. The ID surface contour (as shown in Figure 7.5) is significantly smoother than those of the WOG specimens described earlier. Although this specimen exhibits shallow counterbore facets, the ID surface conditions are relatively smooth, with more fluid contours between the weld root, counterbore, and parent material. The crack in this pipe section was created using laboratory methods developed at PNNL that have proven useful in producing realistic surface-connected thermal fatigue cracks. The flaw in this specimen is basically considered to be a planar crack, parallel to the weld centerline and perpendicular to and connected to the ID. This specimen was stamped with an identifying code (B-515) in the upper left corner and contains a scribe line located near, but not necessarily coincident with, the weld centerline, which was used as the reference line for axial offset measurements.

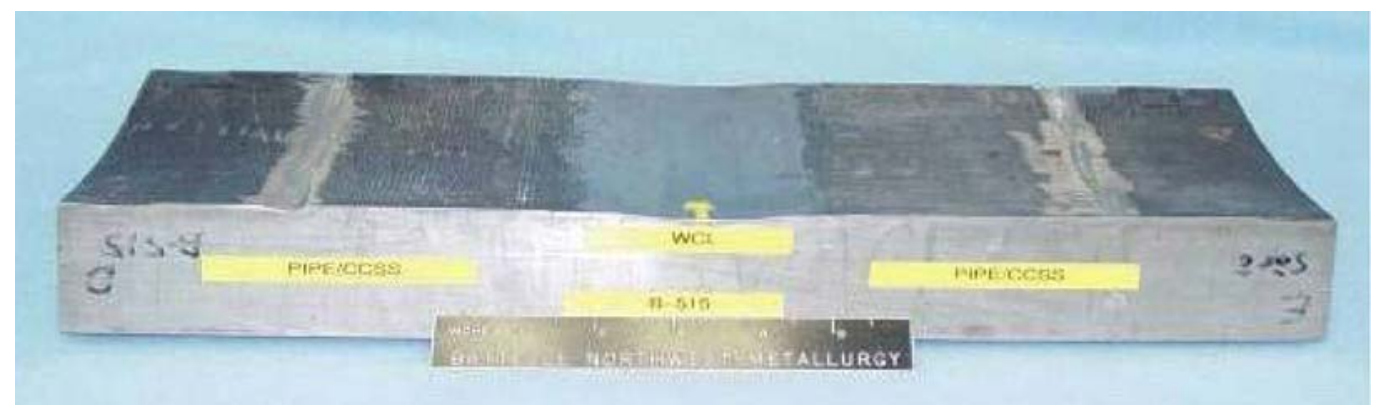

Figure 7.5. Photograph of PNNL CASS Specimen B-515

Specimen \#B-515 is 18.16-cm (7.15-inch) wide (circumferential distance) and 40.4-cm (15.91-inch) long in axial extent. This specimen is configured as a pipe-to-pipe segment with the (-) side consisting of intermediate-size columnar grains and the $(+)$ side consisting of intermediate-size equiaxed grains. The weldment is located approximately in the center of the pipe-to-pipe segment. This specimen has a thermal fatigue crack on the equiaxed $(+)$ side of the weld with an offset of $0.13 \mathrm{~cm}(0.05$ inch $)$ from the weld centerline. The crack is $2.92 \mathrm{~cm}$ (1.15 inch) in length (circumferential extent) and 1.52-cm (0.6-inch) deep. The equiaxed segment of this specimen is 5.84-cm (2.3-inch) thick, and the columnar segment of this specimen is $6.05-\mathrm{cm}(2.38$-inch) thick. The segment thickness at the weld centerline is $5.49 \mathrm{~cm}$ (2.16 inch). This specimen is special in that \#B-515 has been thermally stress-relieved, mechanically bent open, and then bent back to its original shape. This resulted in an open, easier-todetect crack, which may not mimic defects anticipated to be found in service. 


\subsection{Data Analysis and Results: CASS PZR Surge Line}

\subsection{0-kHz Phased Array Data}

The $800-\mathrm{kHz}$ data were acquired with the DYNARAY ${ }^{\circledR}$ system. Line scans were performed on the PZR surge line piece from both the CCSS pipe side and from the SCSS elbow side of the weld as the refracted angle was swept from $30^{\circ}$ to $70^{\circ}$ in $1^{\circ}$ increments. Three line scans were acquired with different axial offsets from the weld centerline. The separation between lines was $15 \mathrm{~mm}$. Data normal to the weld centerline was acquired as well as data at $\pm 10^{\circ}$ and $\pm 20^{\circ}$ skew. To date, only the normal data has been analyzed. The data were merged and analyzed for flaw detection, length and depth sizing, and signal-to-noise information. Additionally, raster scans were acquired on each flaw from the pipe side and from the elbow side of the weld. These files have not yet been analyzed.

Each of the four flaw areas were scanned from the CCSS side of the weld. The inner elbow radius did not allow data collection on flaw 3 from the SCSS, elbow side of the weld. A merged data file analysis setup is shown in Figure 8.1. The B-scan side view is shown in the lower left, the B-scan end view in the lower right, and the C-scan top view in the upper left. The ID corner signal is identified with an arrow in each of the three views.

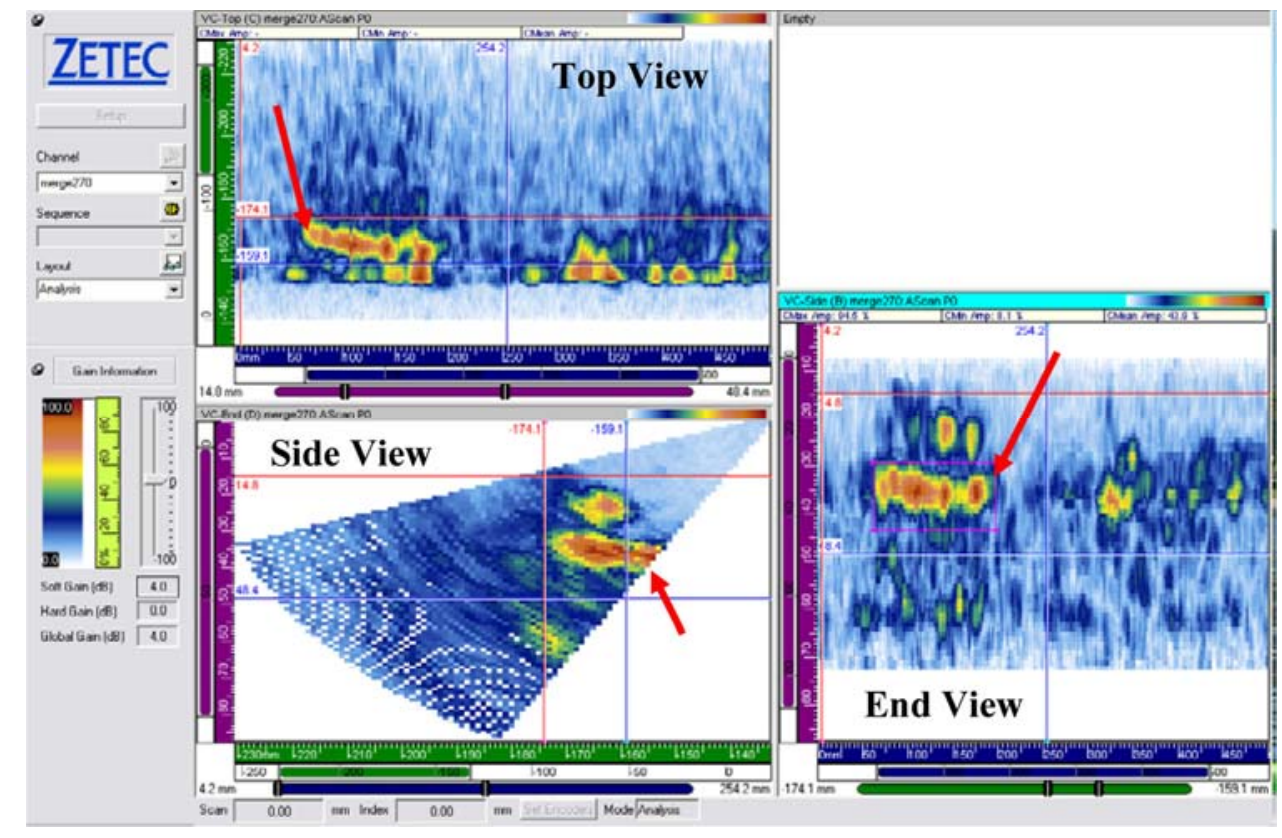

Figure 8.1. PZR Surge Line Flaw 1 from the CCSS Pipe Side at an Axial Position of $-130 \mathrm{~mm}$ and at $800 \mathrm{kHz}$. The corner signal is identified with arrows.

An upper portion of the flaw is also seen in each of the data sets and is identified in Figure 8.2 with arrows in each view. This "top" of the flaw signal is assumed to be a specular reflection and produces a large amplitude response. This is not a tip-diffracted signal which would be expected to be approximately 
ten times $(-20 \mathrm{~dB})$ lower in amplitude than a corner response. These flaw top signals are similar to or within $73 \%$ of the corner response $(0$ to $-2.7 \mathrm{~dB})$ in amplitude. The upper portion of the flaw is boxed in the side and end views to give an isolated view of this top flaw signal in the $\mathrm{C}$-scan top view. In other words, the corner signal is removed in the C-scan top view.

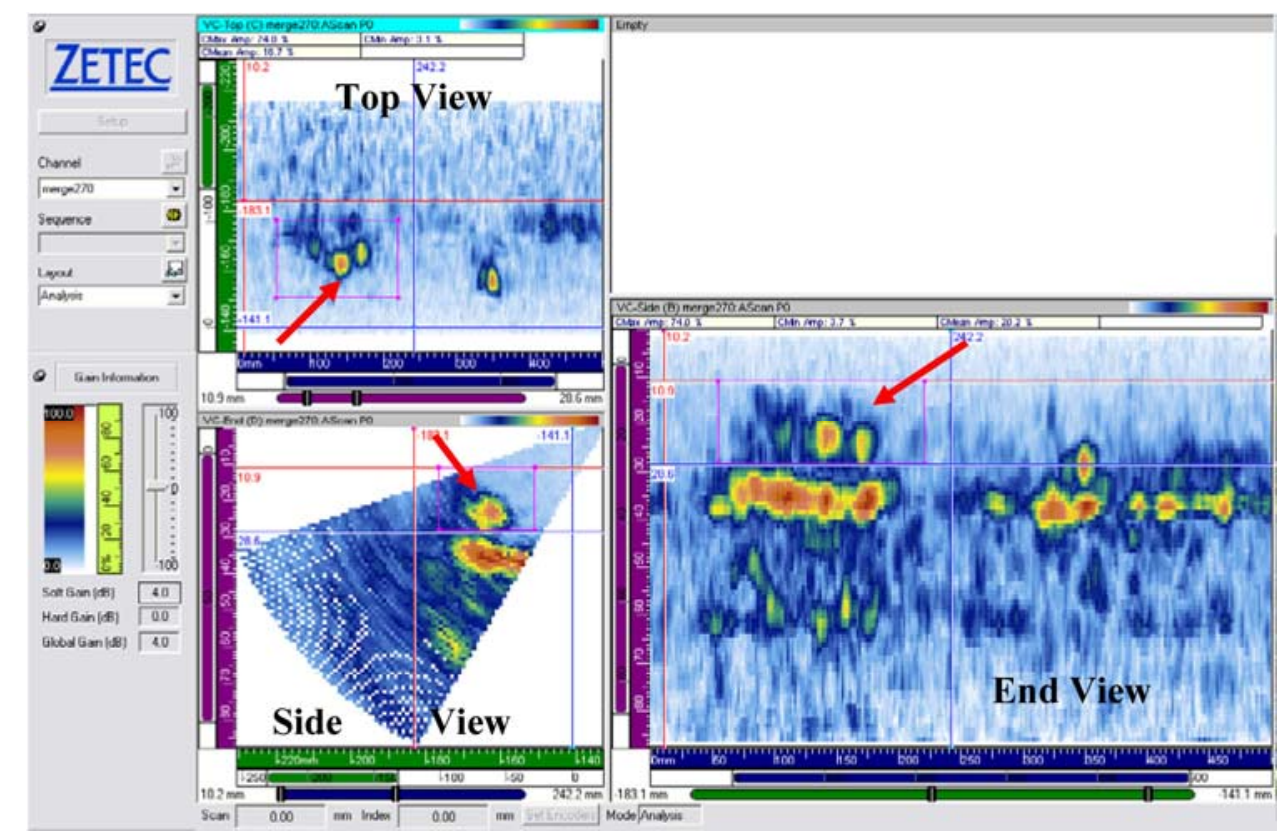

Figure 8.2. PZR Surge Line Flaw 1 from the CCSS Pipe Side at an Axial Position of $-130 \mathrm{~mm}$. The upper portion of the flaw is boxed in the side and end views providing an isolated view of the upper portion of the flaw in the C-scan, top view.

The merged data images for each of the flaws from the CCSS and SCSS sides are shown in Appendix A. The data set with an axial offset that best positioned the flaw response in the center of the side view was analyzed and is also shown in the appendix. Both the corner signal in one figure and an isolated tip signal in a second figure are shown for each of the detected flaws.

As part of the data analysis, the flaw length and depth were sized and a signal-to-noise determination was made. A flaw length was measured at the half amplitude (-6 dB) points from both the flaw corner signal and the flaw top signal since both signals were strong. The results are listed in Tables 8.1 and 8.2. Also shown in the tables are the calculated root mean square error (RMSE) values. Both the corner and top signal length measurements show a better result in terms of RMSE from the SCSS side by a factor of two or more.

A flaw depth measurement was made from the center of the corner signal to the center of the top signal at the deepest portion. The results are shown in Table 8.3 with the RMSE also listed. Because the depth is based on a specular reflection from the upper portion of the flaw and not a crack tip, the full extent of the crack depth might not be measured accurately. The results are still quite good and tend to oversize the crack depth, suggesting that indeed the top of the crack was detected. 
Table 8.1. PZR Surge Line Flaw Length Sizing by Corner Signal at $800 \mathrm{kHz}$

\begin{tabular}{cccc}
\hline Flaw Number & $\begin{array}{c}\text { True Length, } \\
\text { mm (inch) }\end{array}$ & $\begin{array}{c}\text { CCSS Side, } \\
\text { mm (inch) }\end{array}$ & $\begin{array}{c}\text { SCSS Side, } \\
\text { mm (inch) }\end{array}$ \\
\hline 1 & $104(4.09)$ & $122(4.80)$ & $88(3.46)$ \\
2 & $51(2.01)$ & $52(2.05)$ & $54(2.13)$ \\
3 & $50(1.97)$ & $56(2.20)$ & -- \\
4 & $152(5.98)$ & $78(3.07)$ & $132(5.20)$ \\
\hline RMSE CCSS: 38 mm (1.5 in.); SCSS: 15 mm (0.59 in.) & \\
\hline
\end{tabular}

Table 8.2. PZR Surge Line Flaw Length Sizing by Top Signal at $800 \mathrm{kHz}$

\begin{tabular}{cccc}
\hline Flaw Number & $\begin{array}{c}\text { True Length, } \\
\text { mm (inch) }\end{array}$ & $\begin{array}{c}\text { CCSS Side, } \\
\text { mm (inch) }\end{array}$ & $\begin{array}{c}\text { SCSS Side, } \\
\text { mm (inch) }\end{array}$ \\
\hline 1 & $104(4.09)$ & $80(3.15)$ & $78(3.07)$ \\
2 & $51(2.01)$ & $26(1.02)$ & $19(0.75)$ \\
3 & $50(1.97)$ & $50(1.97)$ & -- \\
4 & $152(5.98)$ & $32(1.26)$ & $121(4.76)$ \\
\hline RMSE CCSS: $63 \mathrm{~mm}$ (2.48 in.); SCSS: $30 \mathrm{~mm}(1.18$ in.) \\
\hline
\end{tabular}

Table 8.3. PZR Surge Line Flaw Depth Sizing at $800 \mathrm{kHz}$

\begin{tabular}{cccc}
\hline Flaw Number & $\begin{array}{c}\text { True Depth, } \\
\text { mm (inch) }\end{array}$ & $\begin{array}{c}\text { CCSS Side, } \\
\text { mm (inch) }\end{array}$ & $\begin{array}{c}\text { SCSS Side, } \\
\text { mm (inch) }\end{array}$ \\
\hline 1 & $10.9(0.43)$ & $12.8(0.50)$ & $11.9(0.47)$ \\
2 & $9.3(0.37)$ & $8.9(0.35)$ & $14.8(0.58)$ \\
3 & $9.3(0.37)$ & $12.8(0.50)$ & -- \\
4 & $15.6(0.61)$ & $10.9(0.43)$ & $12.9(0.51)$ \\
\hline RMSE CCSS: 3.1 mm (0.12 in.); SCSS: $3.6 \mathrm{~mm}(0.40$ in.) & \\
\hline
\end{tabular}

Signal-to-noise ratios (SNR) were calculated for the corner signals and the top or upper portion of the flaw signals from the CCSS and SCSS sides. The SNR was determined from the peak signal response and an average noise value at the same part path. Results are listed in Table 8.4 for the $800-\mathrm{kHz}$ data. Also listed are the average SNR values. In this data the top flaw signals are strong with less background noise, likely due to a shorter metal path and therefore less attenuation and scattering by the large grains. The data shows an approximately 4-7 dB greater SNR in the top flaw signal than the corner signal. 
Table 8.4. PZR Surge Line Flaw Signal-to-Noise Ratios at $800 \mathrm{kHz}$

\begin{tabular}{ccccc}
\hline Flaw Number & CCSS Corner, dB & CCSS Top, dB & SCSS Corner, dB & SCSS Top, dB \\
\hline 1 & 14.1 & 18.3 & 7.9 & 23.0 \\
2 & 12.2 & 17.0 & 11.0 & 13.5 \\
3 & 12.9 & 15.0 & -- & -- \\
4 & 11.4 & 17.7 & 17.5 & 22.1 \\
Average & 12.6 & 17.0 & 12.1 & 19.5 \\
\hline
\end{tabular}

\subsection{5-MHz Phased Array Data}

The 1.5-MHz data were acquired with the standard Tomoscan ${ }^{\circledR}$ III system. Line scans were performed on the surge-line piece from both the CCSS pipe side and from the SCSS elbow side of the weld as the refracted angle was swept from $30^{\circ}$ to $70^{\circ}$ in $1^{\circ}$ increments. Two line scans were acquired with different axial offsets from the weld centerline. The separation between lines was $10 \mathrm{~mm}$. Only $90^{\circ}$ skewed data were acquired; this positions the probe normal to the weld centerline. Merged data were analyzed for flaw detection, length and depth sizing, and signal-to-noise information.

As with the $800-\mathrm{kHz}$ data, the data file at the axial offset that best positioned the flaw corner signal and top signal reflectors in the middle of the B-scan side view were selected for the $1.5-\mathrm{MHz}$ data analysis. Data were acquired on the four flaws from the CCSS side of the weld and from flaws 1, 2, and 4 on the SCSS side. The elbow curvature did not allow scanning in the region of flaw 3 from the SCSS side. Images from these seven data files are shown in Appendix B and show both the corner reflectors and top of flaw reflectors. Generally the corner reflector was equal to or greater than the top signal. Two exceptions were found in the CCSS data on flaws 2 and 4 . Figure 8.3 shows the data from a scan that captured both flaws 3 and 4 . Flaw 3 is on the left in the end view and top view and flaw 4 is on the right. The top signal from flaw 3 is $3.8 \mathrm{~dB}$ greater than the corner signal; whereas, the top signal from flaw 4 is $2.7 \mathrm{~dB}$ lower than the corner signal. 


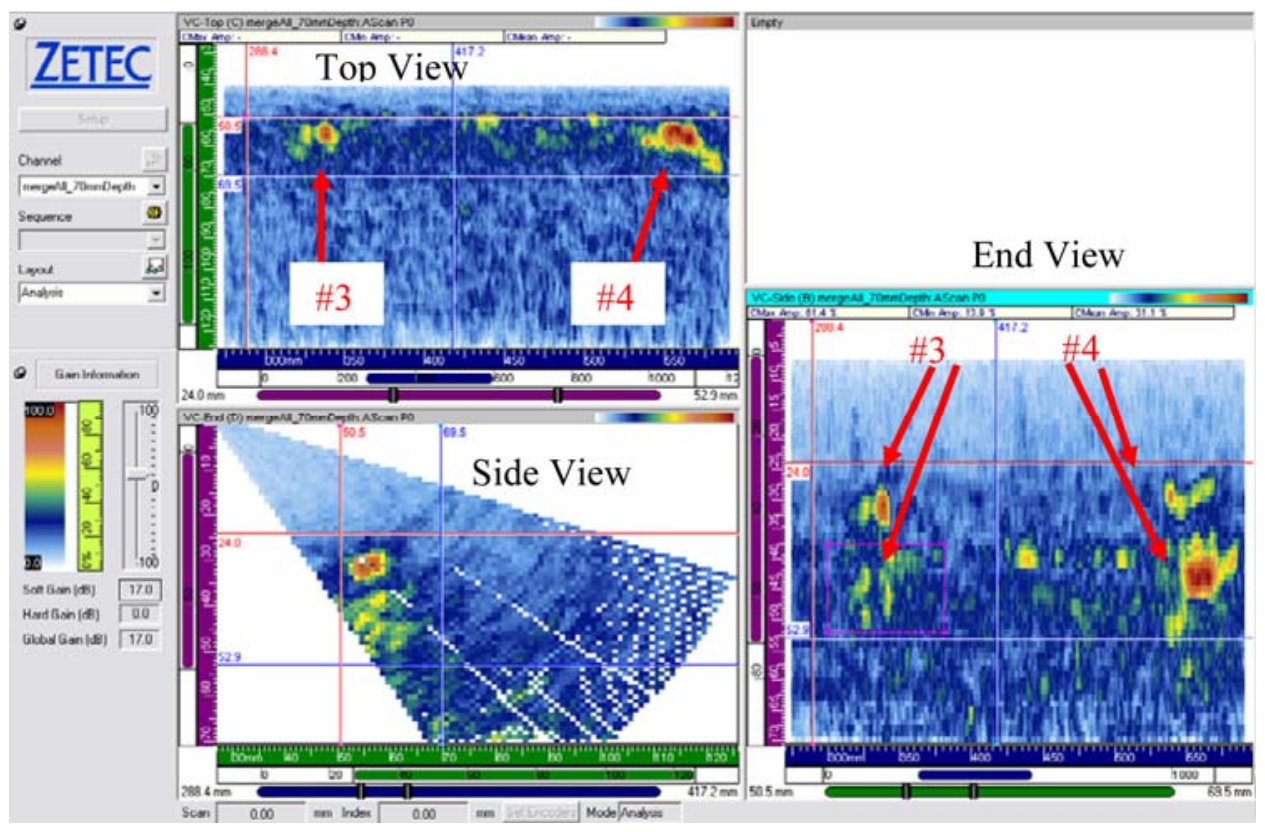

Figure 8.3. A Strong Corner Response from Flaw 4 is Shown in the Data and is More Typical of Flaw Responses. Flaw 3 has a strong top of flaw signal and exhibits a weaker corner signal.

Flaw length, depth, and signal-to-noise were determined from the data. Tables 8.5 and 8.6 show the length results from the corner signals and top of flaw signals and the RMSE calculated from the CCSS pipe side and the SCSS elbow side. At $1.5 \mathrm{MHz}$, the length sizing is slightly better (by approximately $20 \%$ ) from the SCSS side of the weld than from the CCSS side. Additionally, these limited data show a better length sizing performance at $1.5 \mathrm{MHz}$ as opposed to $800 \mathrm{kHz}$. This may in fact be attributed to the smaller spot size of the zone-focused insonification area for the $1.5-\mathrm{MHz}$ probe over the $800-\mathrm{kHz}$ probe.

Table 8.5. PZR Surge Line Flaw Length Sizing by Corner Signal at $1.5 \mathrm{MHz}$

\begin{tabular}{cccc}
\hline Flaw Number & $\begin{array}{c}\text { True Length, } \\
\text { mm (inch) }\end{array}$ & $\begin{array}{c}\text { CCSS Side, } \\
\text { mm (inch) }\end{array}$ & $\begin{array}{c}\text { SCSS Side, } \\
\text { mm (inch) }\end{array}$ \\
\hline 1 & $104(4.09)$ & $111(4.37)$ & $83(3.27)$ \\
2 & $51(2.01)$ & $37(1.46)$ & $72(2.83)$ \\
3 & $50(1.97)$ & $40(1.57)$ & -- \\
4 & $152(5.98)$ & $190(7.48)$ & $161(6.34)$ \\
\hline RMSE CCSS: $21.1 \mathrm{~mm}(0.83$ in.); SCSS: $17.9 \mathrm{~mm}(0.70$ in.) & \\
\hline
\end{tabular}


Table 8.6. PZR Surge Line Flaw Length Sizing by Top Signal at $1.5 \mathrm{MHz}$

\begin{tabular}{cccc}
\hline Flaw Number & $\begin{array}{c}\text { True Length, } \\
\text { mm (inch) }\end{array}$ & $\begin{array}{c}\text { CCSS Side, } \\
\text { mm (inch) }\end{array}$ & $\begin{array}{c}\text { SCSS Side, } \\
\text { mm (inch) }\end{array}$ \\
\hline 1 & $104(4.09)$ & $76(2.99)$ & $72(2.83)$ \\
2 & $51(2.01)$ & $25(0.98)$ & $51(2.01)$ \\
3 & $50(1.97)$ & $38(1.50)$ & -- \\
4 & $152(5.98)$ & $128(5.04)$ & $157(6.18)$ \\
\hline RMSE CCSS: $23.3 \mathrm{~mm}(0.92$ in.); SCSS: & $18.7 \mathrm{~mm}(0.74$ in.) & \\
\hline
\end{tabular}

The flaw depth measurements are shown in Table 8.7. The RMSE values are similar from either the CCSS side or the SCSS side of the weld. A tendency to oversize the flaw depth is evident as was also seen in the $800-\mathrm{kHz}$ results.

Table 8.7. PZR Surge Line Flaw Depth Sizing at $1.5 \mathrm{MHz}$

\begin{tabular}{cclc}
\hline Flaw Number & $\begin{array}{c}\text { True Depth, } \\
\text { mm (inch) }\end{array}$ & $\begin{array}{c}\text { CCSS Side, } \\
\text { mm (inch) }\end{array}$ & $\begin{array}{c}\text { SCSS Side, } \\
\text { mm (inch) }\end{array}$ \\
\hline 1 & $10.9(0.43)$ & $13(0.51)$ & $12(0.47)$ \\
2 & $9.3(0.37)$ & $12(0.47)$ & $10(0.39)$ \\
3 & $9.3(0.37)$ & $13.5(0.53)$ & -- \\
4 & $15.6(0.61)$ & $16(0.63)$ & $11(0.43)$ \\
\hline RMSE CCSS: $2.7 \mathrm{~mm}(0.11$ in.); SCSS: $2.8 \mathrm{~mm}(0.11$ in.) & \\
\hline
\end{tabular}

Signal-to-noise ratios are listed in Table 8.8 for the 1.5-MHz data. Again, the top of flaw signals show a better SNR than the corner signals.

Table 8.8. PZR Surge Line Flaw Signal-to-Noise Ratios at $1.5 \mathrm{MHz}$

\begin{tabular}{ccccc}
\hline Flaw Number & CCSS Corner, dB & CCSS Top, dB & SCSS Corner, dB & SCSS Top, dB \\
\hline 1 & 11.0 & 14.0 & 18.7 & 20.9 \\
2 & 6.8 & 13.6 & 13.9 & 17.7 \\
3 & 13.9 & 12.1 & -- & -- \\
4 & 11.7 & 18.4 & 14.6 & 19.9 \\
Average & 10.9 & 14.5 & 15.8 & 19.5 \\
\hline
\end{tabular}




\subsection{Data Analysis and Results: WOG Specimens}

Data from three WOG specimens were acquired with the $800-\mathrm{kHz}$ phased array transducers. The purpose was to evaluate the probe and the new DYNARAY ${ }^{\circledR}$ system performance on thicker CCSS/SCSS welded assemblies. The three specimens were ONP-3-5, OPE-5, and MPE-6; each contained a thermal fatigue crack located on the SCSS side of the weld centerline. The through-wall crack depth for these three specimens was $28 \%, 18 \%$, and $23 \%$, respectively, as reported by EPRI. The approximate crack area was $11.7 \mathrm{~cm}^{2}, 10 \mathrm{~cm}^{2}$, and $8.8 \mathrm{~cm}^{2}$, respectively.

Data were acquired over refracted angles of $30^{\circ}$ to $60^{\circ}$ with one degree increments in line scans. The probe was positioned normal to the weld centerline and scanned circumferentially. Where possible, several line scans were acquired on a specimen with $12.7-\mathrm{mm}(0.5$-inch) separation in the axial direction between scans and on both the CCSS and SCSS sides of the weld. Each line scan contained normal data and data at $\pm 10^{\circ}$ skew. Additionally, raster data were acquired on MPE- 6 and OPE-5. Only the normal line scan data were analyzed for this TLR.

Figure 9.1 shows the results from the $800-\mathrm{kHz}$ inspection on MPE-6 from the SCSS side. Appendix $\mathrm{C}$ contains all the $800-\mathrm{kHz}$ data images.

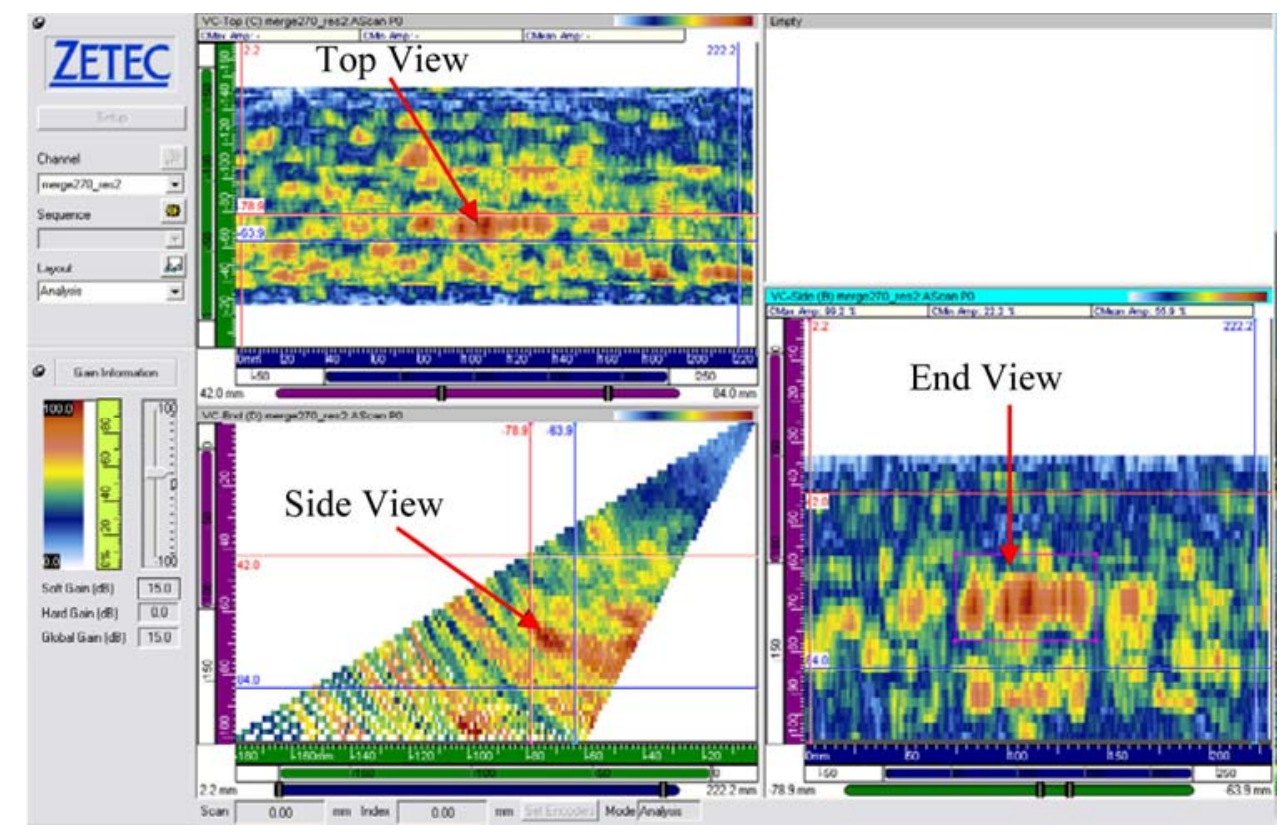

Figure 9.1. Thermal Fatigue Crack is Detected in MPE-6; 800-kHz Data from the SCSS Side of the Weld

A length sizing summary is shown in Table 9.1 for these three WOG specimens. None of the probes were able to detect the crack in ONP-3-5. Crack tip signals were not detected. This is the primary justification for continued development of state-of-the-art lower-frequency $(500 \mathrm{kHz}) \mathrm{PA}$ probes for these thicker specimens where the microstructures preclude effective penetration and resolution. 
Table 9.1. Length Sizing of WOG Specimens with Phased Array at Various Inspection Frequencies

\begin{tabular}{lccc}
\hline & & \multicolumn{2}{c}{ Length, mm (inch) } \\
\cline { 3 - 4 } WOG Specimen & Side & True & $\mathbf{8 0 0} \mathbf{~ k H z}$ \\
\hline ONP-3-5 & CCSS & $66(2.60)$ & ND \\
OPE-5 & CCSS & $61(2.40)$ & $74(2.91)$ \\
& SCSS & $61(2.40)$ & $43(1.69)$ \\
MPE-6 & CCSS & $59(2.32)$ & $36(1.42)$ \\
& SCSS & $59(2.32)$ & $62(2.44)$ \\
\hline ND = Not Detected & & & \\
\hline
\end{tabular}




\subsection{Data Analysis and Results: Delta-Technique (Tip Diffraction)}

The component specimens used for evaluation of the PA delta-technique included:

- Pressurizer Surge Line: Flaws 1, 2, and 4.

- Wrought stainless steel 10\%, 20\%,30\%, and 40\% EDM through-wall notches.

- WOG OPE-5 with a 2.42-inch long, 0.64-inch deep (29.8\% through-wall) thermal fatigue crack.

- CASS pipe segment B-515 (used only the parent-material of this PNNL specimen).

Several probe positions relative to the weld centerline or flaw were evaluated for the delta-technique examinations. The transmitter and receiver were placed equidistant from the feature of interest as illustrated in Figure 10.1, with probe spacings of 0.4 inches $(10.2 \mathrm{~mm})$ to 0.8 inches $(20.3 \mathrm{~mm})$; the receiver was positioned closer to or farther from the feature of interest while preserving the probe separation; or the transmitter or receiver were placed immediately over the item of interest. Scans with the probes positioned equidistant from the item of interest, or with the receiver slightly closer to the item of interest, yielded the best results (Figure 10.2). Scans with the receiver positioned directly over the item of interest yielded marginal results (see Figure 10.3), and scans with the transmitter positioned closer to or directly over the item of interest yielded no valuable data.

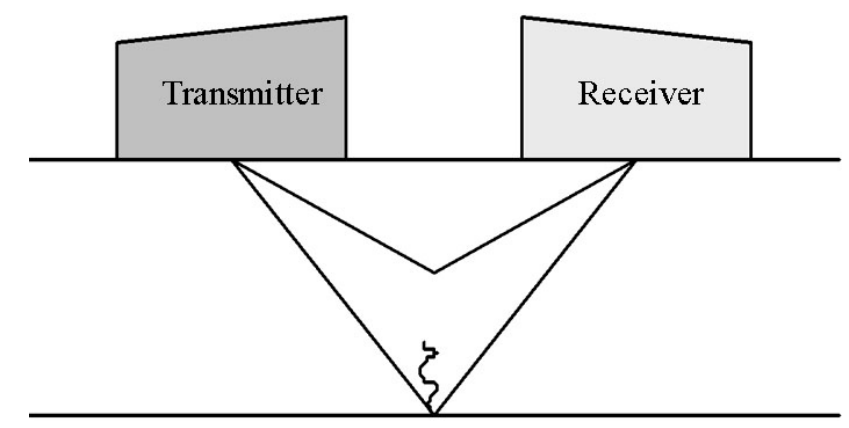

Figure 10.1. Example of Probes Positioned Equidistant from Weld Centerline or Flaw

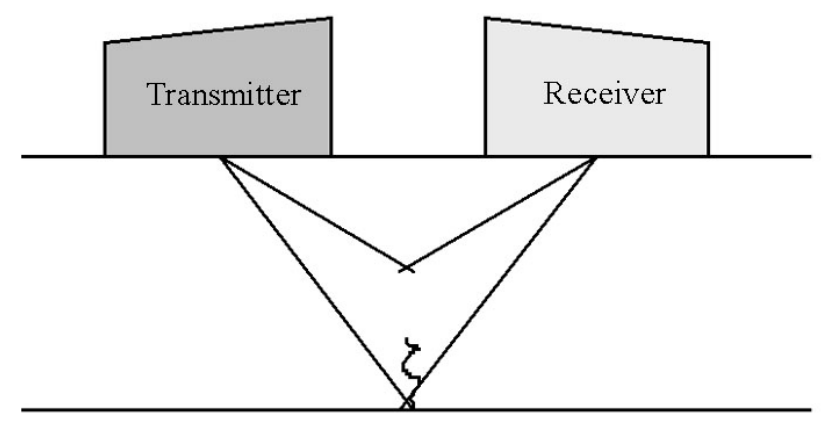

Figure 10.2. Example of Receiver Positioned Slightly Closer to the Weld Centerline or Flaw 


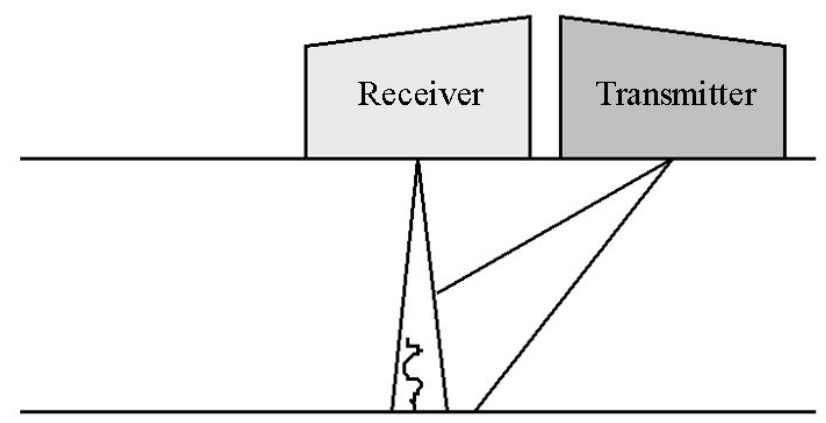

Figure 10.3. Example of Receiver Positioned Directly Over the Weld Centerline or Flaw

The delta technique as applied here basically captures a strong reflection off the backwall of the specimen unless this sound path is blocked by a flaw. Additionally a weak flaw tip-diffracted signal is possibly detected in the flaw area. The loss of backwall signal was primarily used to identify a flaw region and by gating the region of interest, a tip signal was detected for mid-wall or greater notch depths.

Depth sizing of the EDM notches or thermal fatigue cracks was performed in the composite volumetric merge view of the scanned data sets. This view was chosen for sizing analysis in order to avoid bias that can potentially occur when analyzing unmerged data due to selecting tip depths or backwall depths at different angles. In the volumetric merged view, the centers of the echo dynamic lobes for both the backwall and the tip signals were used for depth sizing of the flaw. See Figure 10.4 for a depth sizing image for the WSS 40\% EDM through-wall notch example.

Length sizing of the EDM notches or thermal fatigue cracks was performed at the $-6 \mathrm{~dB}$ drops along the peak of the backwall signal. Again the flaw was located by a loss of backwall signal. See Figure 10.4 as an example from the WSS 40\% EDM through-wall notch data. 


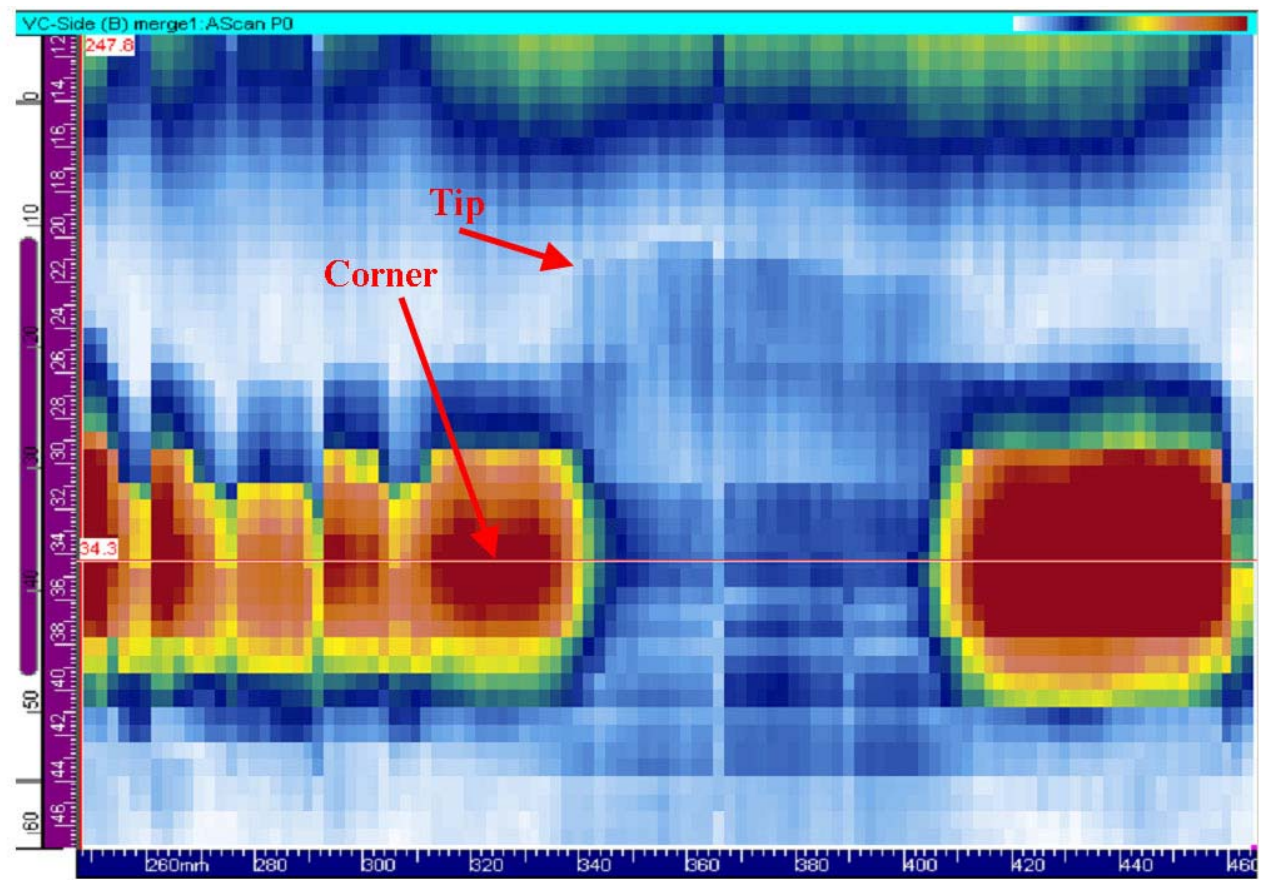

Figure 10.4. End-view from the Wrought Stainless Steel 40\% EDM Notch Scan. The tip diffracted and backwall signals are marked. Notice the lack of backwall signal in the flaw area.

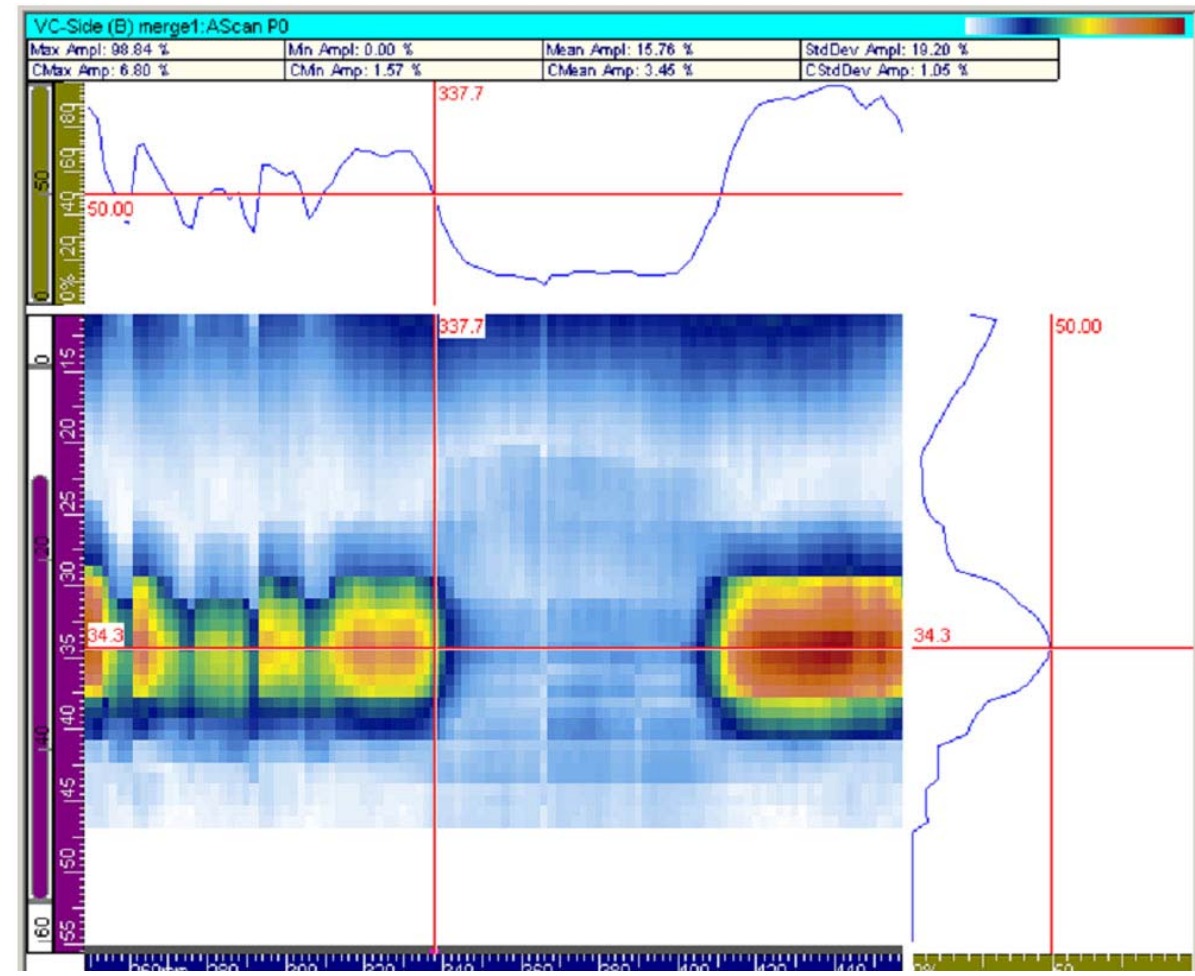

Figure 10.5. Notch Length Determination from the Wrought Stainless Steel 40\% EDM Notch Data as Determined by Loss of Backwall Signal 
The pressurizer surge line B-scans and the remaining wrought stainless steel B-scan views, which were analyzed for depth, length, and signal-to-noise information, can be found in Appendix D. Note that the $10 \%$ wrought stainless steel EDM notch was not detected using the delta technique. OPE-5 and PNNL specimen B-515 were also scanned using the delta technique. The $29.8 \%$ through-wall thermal fatigue crack in OPE- 5 was undetected using this method, and there were no discernible differences between the scans of OPE-5 and the parent material of specimen B-515. B-scan images from the OPE-5 specimen and from B-515 can be found in Appendix D. Tables 10.1 through 10.6 summarize the depth sizing, length sizing, and signal-to-noise values from the EDM notches and thermal fatigue cracks for the wrought stainless steel and pressurizer surge line specimen, respectively. The RMSE has been calculated for the depth and length values and signal-to-noise had been calculated for the tip signals and backwall drop out. For convenience, depth, length, and RMS error values are reported in both metric and English units.

Table 10.1. Wrought Stainless Steel EDM Notch Sizing (in mm)

\begin{tabular}{lcccc}
\hline EDM Notch & $\begin{array}{c}\text { Measured } \\
\text { Tip Depth, } \\
\text { mm }\end{array}$ & $\begin{array}{c}\text { Reported } \\
\text { Depth of } \\
\text { Tip, mm }\end{array}$ & $\begin{array}{c}\text { Measured } \\
\text { Length, mm }\end{array}$ & $\begin{array}{c}\text { True State } \\
\text { Length, } \\
\text { mm }\end{array}$ \\
\hline 40\% Through Wall & 11.4 & 10.2 & 71.12 & 63.5 \\
30\% Through Wall & 9.9 & 7.62 & 71.12 & 63.5 \\
20\% Through Wall & 8.1 & 5.08 & 71.12 & 63.5 \\
10\% Through Wall & Undetected & 2.54 & -- & -- \\
RMS Error (mm) & 2.29 & & 7.62 & \\
\hline
\end{tabular}

Table 10.2. Wrought Stainless Steel EDM Notch Sizing (in inches)

\begin{tabular}{lcccc}
\hline EDM Notch & $\begin{array}{c}\text { Measured } \\
\text { Tip Depth, } \\
\text { in. }\end{array}$ & $\begin{array}{c}\text { Reported } \\
\text { Depth of } \\
\text { Tip, in. }\end{array}$ & $\begin{array}{c}\text { Measured } \\
\text { Length, in. }\end{array}$ & $\begin{array}{c}\text { True State } \\
\text { Length, in. }\end{array}$ \\
\hline 40\% Through Wall & 0.45 & 0.40 & 2.8 & 2.5 \\
30\% Through Wall & 0.39 & 0.30 & 2.8 & 2.5 \\
20\% Through Wall & 0.32 & 0.20 & 2.8 & 2.5 \\
10\% Through Wall & Undetected & 0.10 & -- & 2.5 \\
RMS Error (in.) & 0.091 & & 0.30 & \\
\hline
\end{tabular}

Table 10.3. Wrought Stainless Steel EDM Signal-to-Noise Ratios

\begin{tabular}{lccc}
\hline \multicolumn{1}{c}{ EDM Notch } & $\begin{array}{c}\text { Backwall-to- } \\
\text { Signal Drop } \\
\text { Out Ratio }\end{array}$ & $\begin{array}{c}\text { Tip Signal-to- } \\
\text { Noise Ratio }\end{array}$ & $\begin{array}{c}\text { Backwall-to-Tip } \\
\text { Signal Ratio }\end{array}$ \\
\hline 40\% Through Wall & $8.230(18.3 \mathrm{~dB})$ & $3.70(11.4 \mathrm{~dB})$ & $8.216(18.3 \mathrm{~dB})$ \\
30\% Through Wall & $4.13(12.3 \mathrm{~dB})$ & $2.8(8.9 \mathrm{~dB})$ & $7.41(17.4 \mathrm{~dB})$ \\
20\% Through Wall & $3.26(10.3 \mathrm{~dB})$ & $1.62(4.2 \mathrm{~dB})$ & $3.30(10.4 \mathrm{~dB})$ \\
10\% Through Wall & & Undetected & \\
Average & $5.21(14.3 \mathrm{~dB})$ & $2.71(8.6 \mathrm{~dB})$ & $6.31(16.0 \mathrm{~dB})$ \\
\hline
\end{tabular}


Table 10.4. Pressurizer Surge Line Thermal Fatigue Crack Sizing (in mm)

\begin{tabular}{lcccc}
\hline & $\begin{array}{c}\text { Measured Tip } \\
\text { Depth, } \mathbf{~ m m}\end{array}$ & $\begin{array}{c}\text { True State, } \\
\text { mm }\end{array}$ & $\begin{array}{c}\text { Measured } \\
\text { Length, } \mathbf{~ m m}\end{array}$ & $\begin{array}{c}\text { True State, } \\
\text { mm }\end{array}$ \\
\hline Flaw 1 & 15.3 & 10.9 & 115.9 & 101.6 \\
Flaw 2 & 11.5 & 9.35 & 60.0 & 50.57 \\
& & $9.45-15.6 ;$ & & \\
Flaw 4 & 14.1 & median $=12.5$ & 183.9 & 152.6 \\
RMS Error (mm) & & 3.44 & 24 & \\
\hline
\end{tabular}

Table 10.5. Pressurizer Surge Line Thermal Fatigue Crack Sizing (in inches)

\begin{tabular}{lcccc}
\hline EDM Notch & $\begin{array}{c}\text { Measured Tip } \\
\text { Depth, in. }\end{array}$ & $\begin{array}{c}\text { True State } \\
\text { Depth, in. }\end{array}$ & $\begin{array}{c}\text { Measured } \\
\text { Length, in. }\end{array}$ & $\begin{array}{c}\text { True } \\
\text { State, in. }\end{array}$ \\
\hline Flaw 1 & 0.602 & 0.431 & 4.56 & 3.999 \\
Flaw 2 & 0.453 & 0.368 & 2.36 & 1.991 \\
& & $0.372-0.614 ;$ & & \\
Flaw 4 & 0.555 & median $=0.493$ & 7.24 & 6.006 \\
RMS Error (in.) & 0.116 & & 0.812 & \\
\hline
\end{tabular}

Table 10.6. Pressurizer Surge Line Signal-to-Noise Ratios

\begin{tabular}{lccc}
\hline \multicolumn{1}{c}{ EDM Notch } & $\begin{array}{c}\text { Backwall-to-Signal } \\
\text { Drop Out Ratio }\end{array}$ & $\begin{array}{c}\text { Tip Signal-to-Noise } \\
\text { Ratio }\end{array}$ & $\begin{array}{c}\text { Backwall-to-Tip } \\
\text { Signal Ratio }\end{array}$ \\
\hline Flaw 1 & $12(21.6 \mathrm{~dB})$ & $2.5(8.0 \mathrm{~dB})$ & $4.74(13.5 \mathrm{~dB})$ \\
Flaw 2 & $7.98(18.0 \mathrm{~dB})$ & $12(21.6 \mathrm{~dB})$ & $2.53(8.0 \mathrm{~dB})$ \\
Flaw 4 & $10(20 \mathrm{~dB})$ & $2.6(8.3 \mathrm{~dB})$ & $4.55(13.2 \mathrm{~dB})$ \\
Average & $10(20 \mathrm{~dB})$ & $5.7(15.1 \mathrm{~dB})$ & $3.94(11.9 \mathrm{~dB})$ \\
\hline
\end{tabular}

A primary focus in this study has been in acquiring data on the four thermal fatigue cracks in the PZR surge-line specimen. The three configurations used were the $800-\mathrm{kHz}$ and $1.5-\mathrm{MHz}$ conventional phasedarray technique and the $800-\mathrm{kHz}$ delta technique. On this basis a summary of length and depth sizing results from the four thermal fatigue cracks implanted in the specimen is shown in Figure 10.6 and Figure 10.7. Data were not collected on Flaw 3 with all techniques.

The length sizing calculations are based upon the corner response. The comparison chart shows that the data clusters around the true-state value with one outlier at $800-\mathrm{kHz}$ CCSS (pipe side) on Flaw 4 . The RMSE for length sizing data acquired at $800-\mathrm{kHz}$ CCSS is $38 \mathrm{~mm}$ (1.50 inch), whereas the rest of the data fall in a 15-24 mm (0.59-0.95 inch) RMSE range. According to the ASME Boiler and Pressure Vessel Code, Section XI, Appendix VII, Performance Demonstration for Ultrasonic Examination Systems, the RMSE limit is $19 \mathrm{~mm}(0.75 \mathrm{inch})$. No technique consistently under or oversized the flaw lengths. 


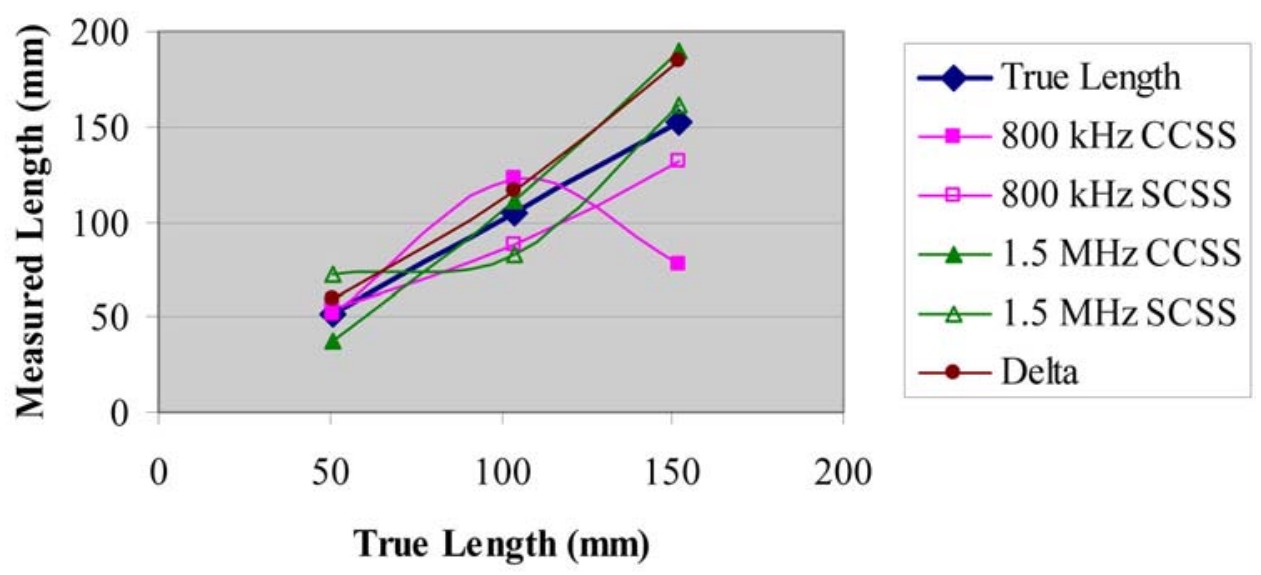

Figure 10.6. Measured Flaw Lengths Versus True Flaw Lengths as a Function of Flaw Number on the PZR Surge-Line Specimen Using All Frequencies and Techniques

The flaw depth comparison in Figure 10.7 shows a possible trend to oversize the shallower three flaws and undersize the deepest flaw, Flaw 4. The RMSE values for these techniques are in the 2.7-3.4 $\mathrm{mm}(0.11-0.13 \mathrm{inch})$ range.

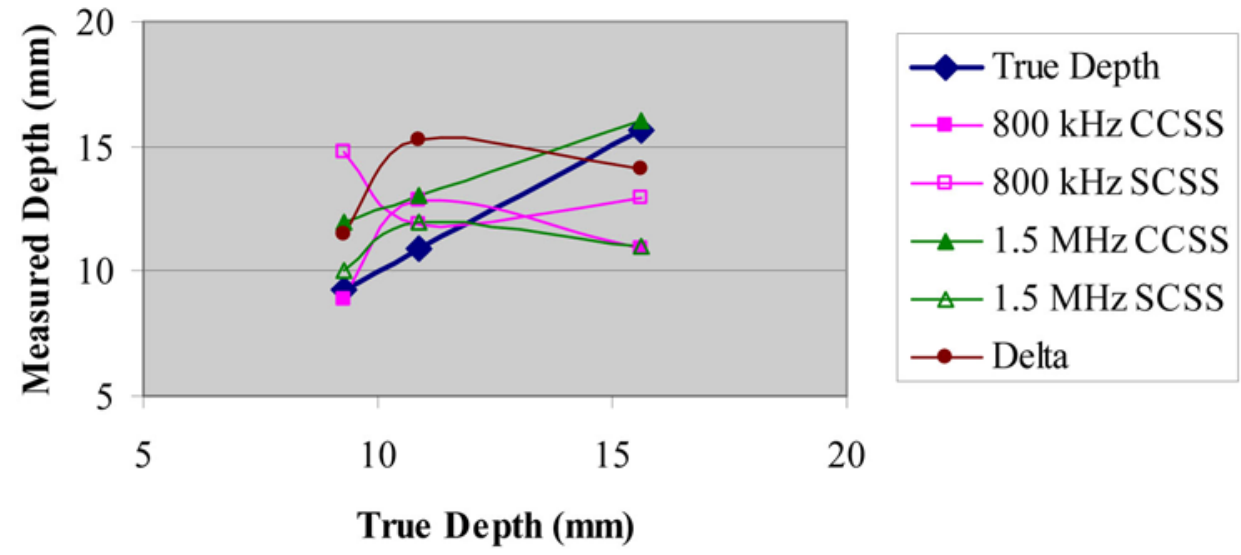

Figure 10.7. Measured Flaw Depths Versus True Flaw Depths as a Function of Flaw Number on the PZR Surge-Line Specimen Using All Frequencies and Techniques 


\subsection{Discussion and Conclusions}

The thermal fatigue cracks in the PZR surge line specimen were detected from both the CCSS and the SCSS sides of the weld at $800 \mathrm{kHz}$ and at $1.5 \mathrm{MHz}$. Data were not collected on one of the cracks from the SCSS elbow side due to geometrical constraints that precluded effective coupling of the PA probe. Length sizing was better from the SCSS side, with a factor of two less error in the $800-\mathrm{kHz}$ data and only slightly less error in the $1.5-\mathrm{MHz}$ data as compared to the CCSS side. The crack depth sizing was similar from either the CCSS or the SCSS side at both frequencies with an approximate 3-mm error. Signal-tonoise values were in the 6.8 to $23 \mathrm{~dB}$ range showing generally good detection of both the crack corner signal and top of flaw signal.

The $800-\mathrm{kHz}$ probe with a wavelength in the material of approximately $7 \mathrm{~mm}(0.28 \mathrm{inch})$ performed well in comparison to the $1.5-\mathrm{MHz}$ probe, which has a wavelength of $4 \mathrm{~mm}(0.16$ inch). A very limited frequency analysis showed that the $800-\mathrm{kHz}$ probe was actually operating at $730-780 \mathrm{kHz}$ in the material due to microstructural "filtering" effects, and the 1.5-MHz probe was effectively operating at 1-1.2 MHz. With both probes approaching a similar frequency range of operation in this thinner material and for these four flaws, it is not surprising that the SNR and depth sizing results are similar. Length sizing differences are attributed to variations in the effective focal spot size of each phased array beam. It should be noted that the microstructures for both the elbow and pipe sections of the PZR surge line were comprised of finer grained microstructures where the average grain diameters were typically smaller than the wavelength of the ultrasonic energy used to inspect this specimen. As discussed in Section 4.0, the specimen evaluated here represents only one type of microstructure, and additional work using the $800-\mathrm{kHz}$ PA probe will be needed to more effectively assess detection and sizing capabilities for PZR surge-line components where the average grain diameters are much larger and where material homogeneity and grain anisotropy will provide more significant inspection challenges.

The limited data comparison from the WOG specimens shows that the $800-\mathrm{kHz}$ probe has the potential for inspecting some of the thicker section coarse-grained materials where attenuation and scattering allow for suitable penetration and resolution. However, it is anticipated that a lower frequency $(500 \mathrm{kHz})$ will be required for many of the dimensionally larger microstructures exhibited (as an example) by the more challenging WOG practice set of specimens that were not evaluated here. The $800-\mathrm{kHz}$ probe was pulsed at its optimum frequency, an 825 nanosecond pulse in this instance. In both the MPE-6 and OPE-5 specimens the center frequency of the flaw signal was greater on the SCSS side than the CCSS side showing that this CCSS material attenuates and scatters more than the SCSS material and also contributes to frequency "filtering" effects. The signal frequency dropped from 810 to $780 \mathrm{kHz}$ in the MPE-6 specimen and from 780 to $680 \mathrm{kHz}$ in the OPE-5 specimen. Again these are preliminary findings based on limited data.

With regard to acquiring baseline data and evaluating the delta-technique for capturing tip-diffracted energy from crack tips in CASS, the 40\%,30\%, and 20\% through-wall EDM notches were detected and depth sized with an RMS error of $2.29 \mathrm{~mm}(0.091 \mathrm{inch})$ and length sized with an RMS error of $7.62 \mathrm{~mm}$ ( 0.30 inch) in the wrought stainless steel specimens. The $10 \%$ through-wall EDM notch was not detected using the delta technique. This is attributed to the size of the wavelength relative to the size of the notch. 
Flaws 1, 2, and 4 on the PZR surge line specimen were detected and depth sized with an RMSE of $3.44 \mathrm{~mm}(0.116 \mathrm{inch})$ and length sized with an RMSE of $24.0 \mathrm{~mm}$ (0.812 inch). Flaw 3 was not scanned using the delta-technique. The flaw sizing tended to be slightly larger than the true state. This "resolution" issue is attributed to the blooming effect that occurs from an incident sound field where the large $7.5-\mathrm{mm}$ wavelength in the material relative to the flaw size results in a distortion of the scattered energy where the resultant image has an exaggerated size, and hence oversized dimension.

The tip-diffraction signals observed from the EDM notches have low to moderate signal-to-noise ratios that range from $1.62(4.2 \mathrm{~dB})$ to $3.70(11.4 \mathrm{~dB})$. The certainty in reporting tip-diffraction signals comes from the significant backwall signal dropout that accompanies these low-amplitude responses, which range from $3.26(10.3 \mathrm{~dB})$ to $8.23(18.3 \mathrm{~dB})$. If the backwall dropout were not evident in the overall images, it would be more challenging to identify and discriminate the tip-diffracted energy from coherent energy scattered from the grain boundaries. As anticipated, the larger the EDM notch (in through-wall depth) the larger the SNR of the tip-diffracted signal for the case of the wrought stainless steel (WSS) coupons. The, tip-diffracted signals observed from the thermal fatigue cracks in the pressurizer surge line were similar to their EDM counterparts, with the exception of Flaw \#2, with signalto-noise ratios ranging from $2.6(8.3 \mathrm{~dB})$ to $12(21.6 \mathrm{~dB})$. The detection and localization of crack tipdiffracted signals is again only made possible from observing a significant backwall signal loss that accompanies these signals, which range from $7.98(18.0 \mathrm{~dB})$ to $12.0(21.6 \mathrm{~dB})$.

The frequency and focusing/steering of the phased array probes used in these delta-technique examinations were not designed to accommodate thin sample materials. Nevertheless, the results obtained in this study demonstrate that there is potential for improving the delta technique by employing optimally designed probes and suitably chosen inspection frequencies. The degree of success, however, is contingent upon the appropriate phased array probe designs, the amount of background scatter from the microstructure, and the ability to capture the backwall signal dropout as a fiduciary for locating/detecting the areas where tip-diffracted energy should be seen. With suitable probes designed for thin materials and more evaluation of appropriate focal laws, the delta-technique approach may prove useful for crack depth determination.

With respect to the evaluations conducted on the CASS PZR surge line and the WOG specimens, the primary issues requiring more research include gaining a better understanding the role of different microstructures in CASS components and the various casting processes, and how variations in these processes and microstructures affect crack initiation, crack growth, and inspection performance. Work on Task 2 is currently focused on this area. An effective and accurate technique for depth sizing fatigue cracks in CASS microstructures is needed as PA probe development continues to advance. Raster and skewed data were acquired at $800 \mathrm{kHz}$ on the PZR surge line specimen and on two of the WOG samples. Analyzing these data could provide additional insights regarding the merit and utility of raster scanning and skewing, particularly on the WOG specimens where flaw detection is difficult. Also, analyzing the $800-\mathrm{kHz}$ raster and skewed data that has already been acquired on the WOG specimens and possibly acquiring data from additional specimens to provide a more substantive set of data from which to base the evaluation may provide better insights into improvements in PA inspection methodologies for CASS. 
While advances in phased-array inspection systems continue to improve general capabilities for crack detection and relatively accurate lateral resolution for length sizing, overcoming the main inspection challenges posed by coarse-grained microstructures requires finding the optimal balance between three key factors. Achieving suitable signal-to-noise ratio, obtaining effective penetration of the sound field from the OD, and acquiring optimal resolution for flaw localization and sizing are all critical toward improving flaw detection and sizing capabilities in CASS.

The work reported here included an initial evaluation of a PA delta-technique for obtaining tipdiffracted signals from cracks in CASS materials. The primary challenge is in consistently and reliably capturing the low-amplitude tip-diffracted energy, and then effectively discriminating those tip-diffracted signals from the coherent energy scattered from the grain boundaries of the microstructure. Once again, the same key factors emerge as unresolved issues that will require more study, and these include balancing the frequency/wavelength issue with suitable signal-to-noise and resolution. Improvements to the probe/wedge design can also be made to enhance the focal characteristics in thinner-walled CASS components. As more information is gained from understanding the microstructures (via casting/fabrication information and direct measurement methods for in-situ microstructure/texture determination), improvements to the inspection parameters can be made more effective, and this in turn should enhance inspection results as well. From this work, it was noted that the potential for a PA "Siamese" imaging method might provide additional or complementary information for crack detection and both length and depth sizing of cracks in these materials. Additional work on PZR surge-line specimens with larger grains and more challenging microstructures is planned in the coming months. 



\subsection{References}

Anderson MT, SL Crawford, SE Cumblidge, KM Denslow, AA Diaz and SR Doctor. 2007. Assessment of Crack Detection in Heavy-Walled Cast Stainless Steel Piping Welds Using Advanced Low-Frequency Ultrasonic Methods. NUREG/CR-6933, PNNL-16292. U.S. Nuclear Regulatory Commission, Washington, DC.

Anderson MT, SE Cumblidge and SR Doctor. 2003. "Applying Ultrasonic Phased Array Technology to Examine Austenitic Coarse-Grained Structures for Light Water Reactor Piping." Third EPRI Phased Array Inspection Seminar. June 9-11, 2003. Seattle, Washington.

Diaz AA, SR Doctor, BP Hildebrand, FA Simonen, GJ Schuster, ES Andersen, GP McDonald and RD Hasse. 1998. Evaluation of Ultrasonic Inspection Techniques for Coarse-Grained Materials. NUREG/CR-6594, PNNL-11171. U.S. Nuclear Regulatory Commission, Washington, DC.

Diaz AA, RA Mathews, J Hixon and SR Doctor. 2007. Assessment of Eddy Current Testing for the Detection of Cracks in Cast Stainless Steel Reactor Piping Components. NUREG/CR-6929, PNNL16253. U.S. Nuclear Regulatory Commission, Washington, DC.

Jeong YH. 1987. An Ultrasonic Material State Classifier for Elastically Anisotropic Materials. Ph.D. Thesis, Drexel University, Philadelphia, Pennsylvania.

Taylor TT. 1984. An Evaluation of Manual Ultrasonic Inspection of Cast Stainless Steel Piping. NUREG/CR-3753, PNL-5070. U.S. Nuclear Regulatory Commission, Washington, DC. 

Appendix A

800-kHz Phased Array Data on the Pressurizer Surge Line 



\section{Appendix A}

\section{0-kHz Phased Array Data on the Pressurizer Surge Line}

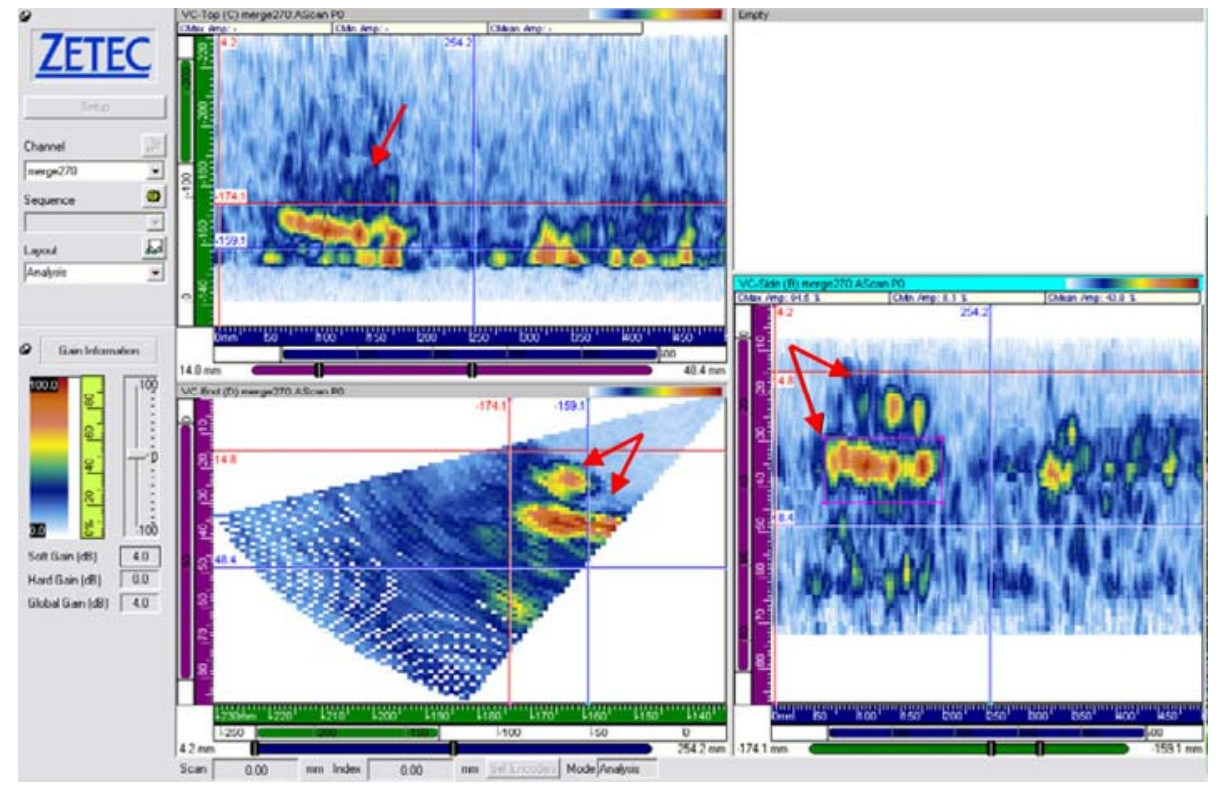

Figure A.1. Surge Line Flaw 1 from the CCSS Pipe Side at $800 \mathrm{kHz}$ and an Axial Position of -130 mm

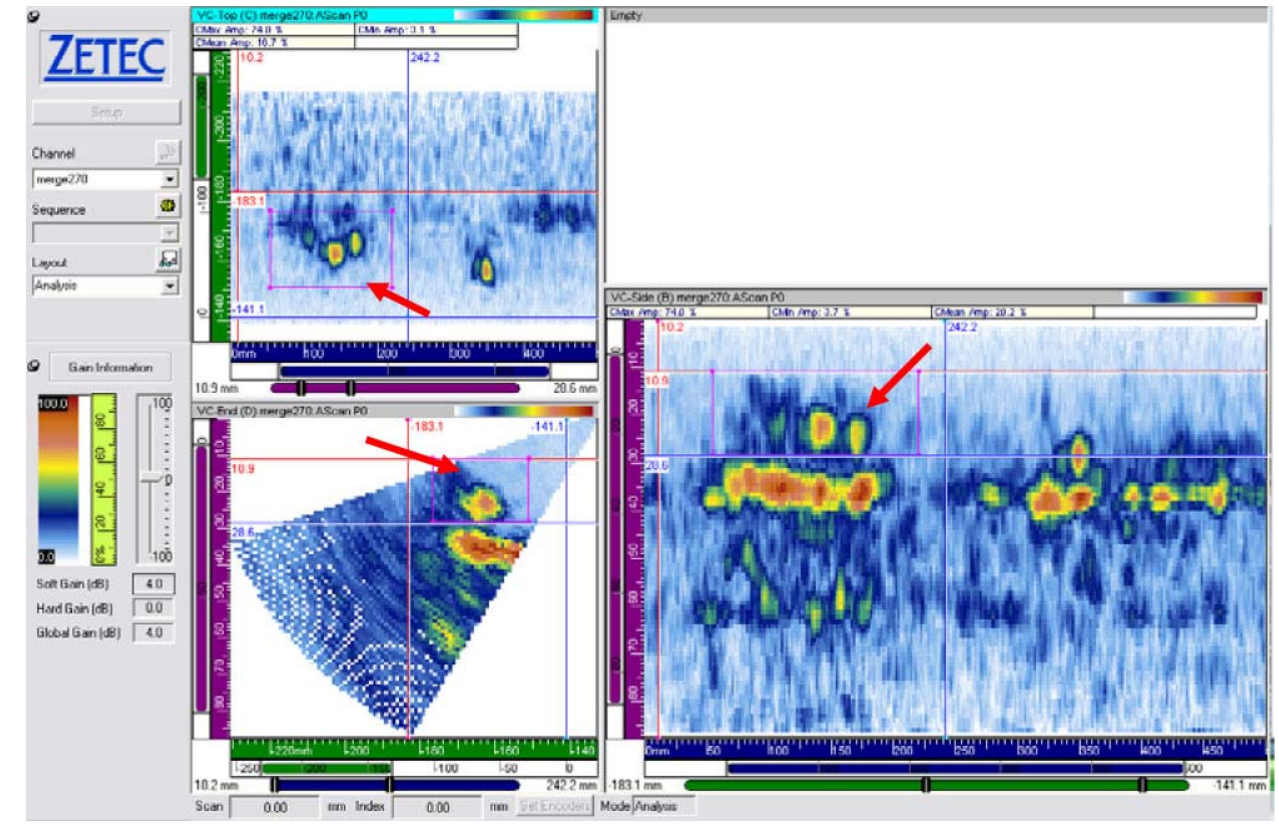

Figure A.2. Surge Line Flaw 1 from the CCSS Pipe Side at $800 \mathrm{kHz}$ and an Axial Position of -130 mm. The upper portion of the flaw is boxed and isolated in the C-scan, top view. 


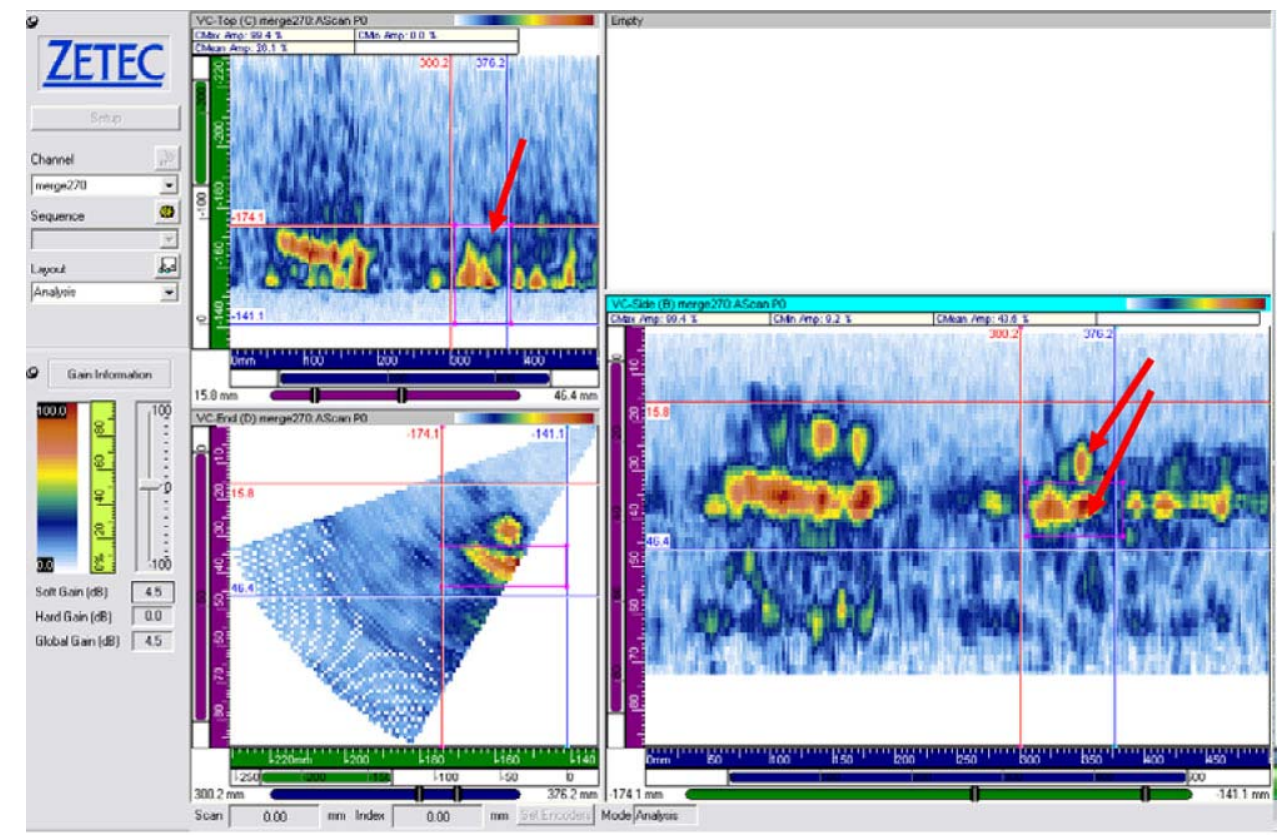

Figure A.3. Surge Line Flaw 2 from the CCSS Pipe Side at $800 \mathrm{kHz}$ and an Axial Position of $-130 \mathrm{~mm}$

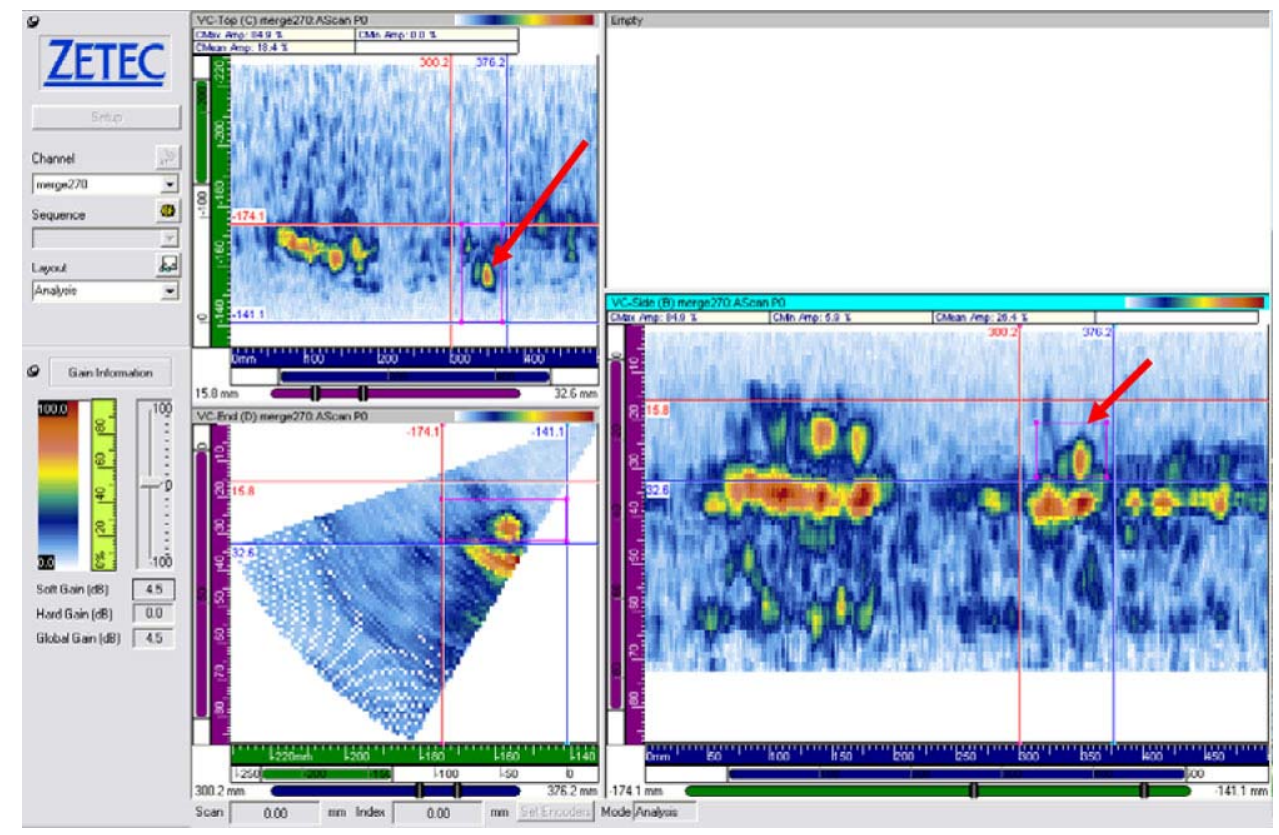

Figure A.4. Surge Line Flaw 2 from the CCSS Pipe Side at $800 \mathrm{kHz}$ and an Axial Position of -130 mm. The upper portion of the flaw is boxed and isolated in the C-scan, top view. 


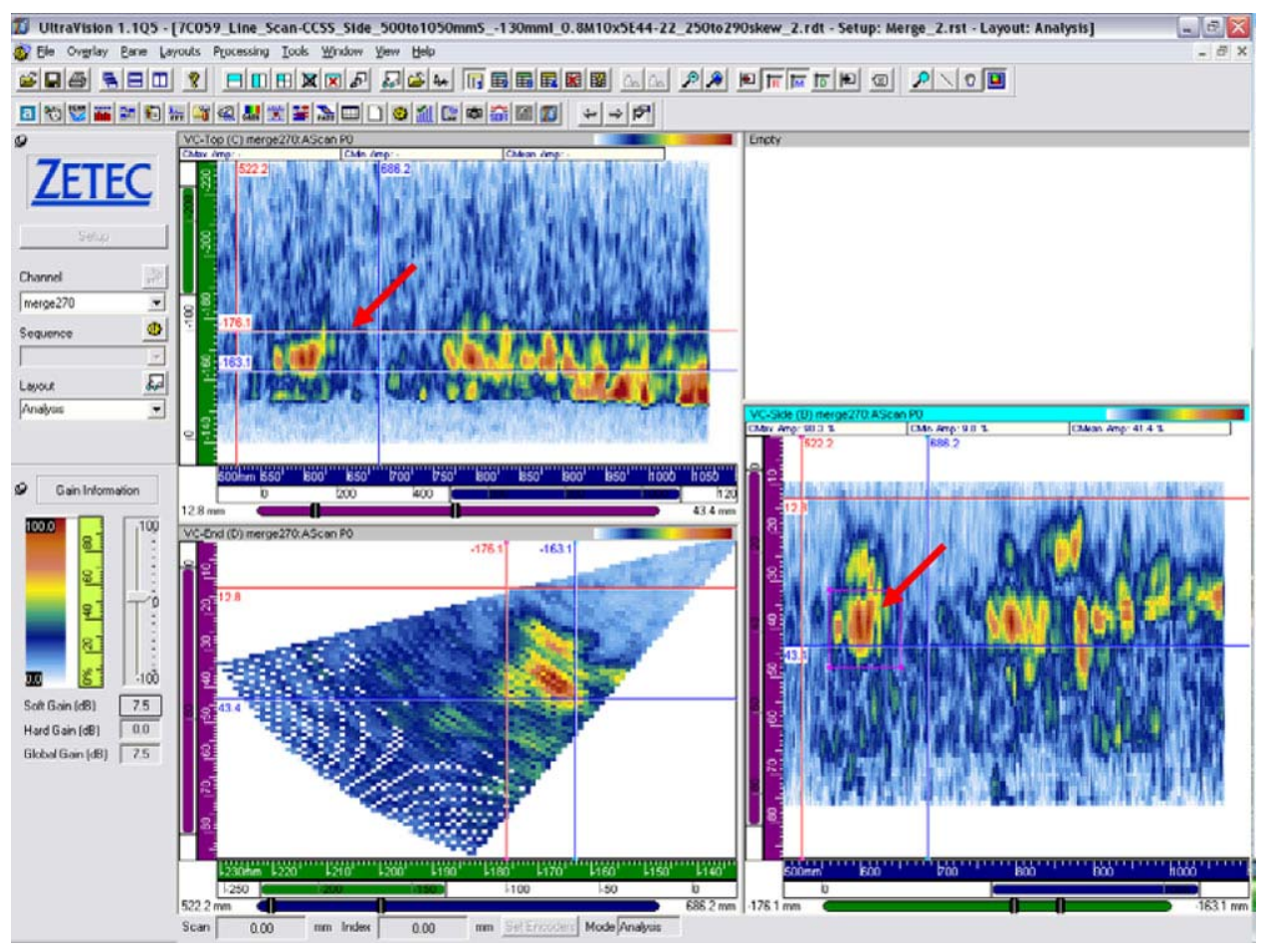

Figure A.5. Surge Line Flaw 3 from the CCSS Pipe Side at $800 \mathrm{kHz}$ and an Axial Position of -130 mm

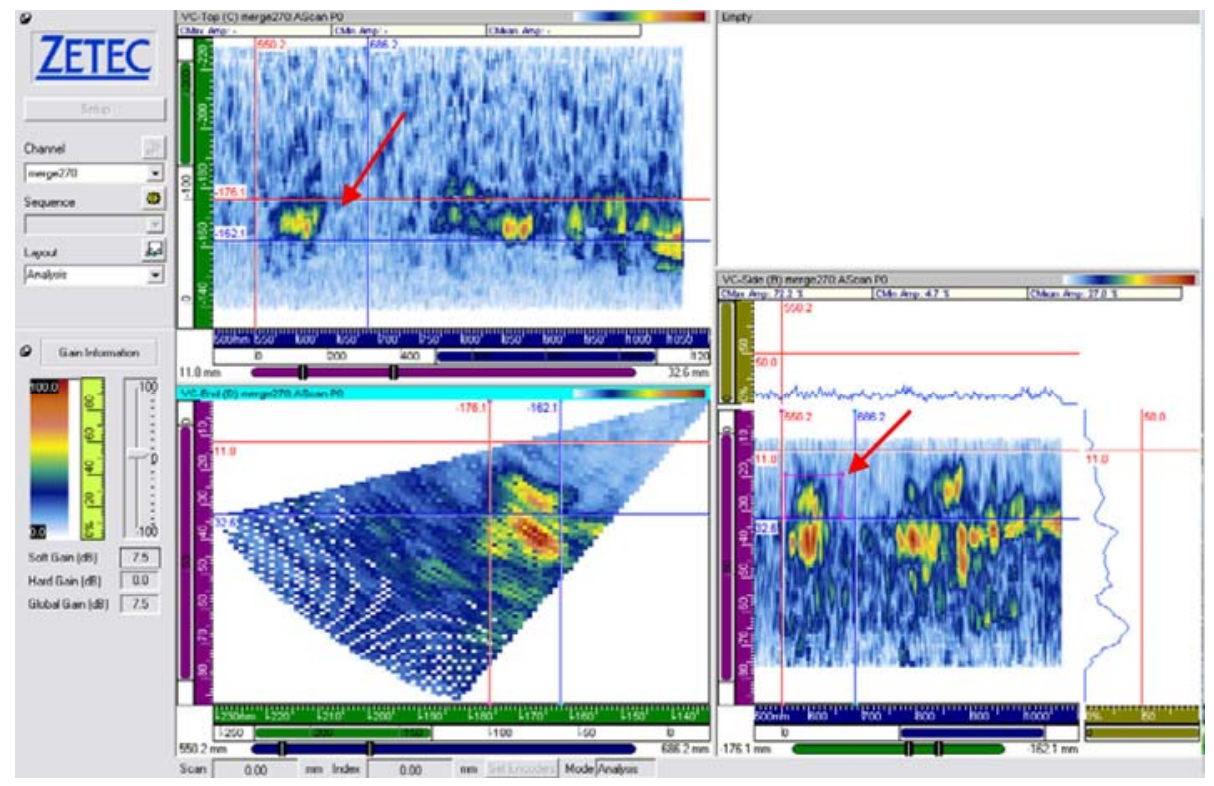

Figure A.6. Surge Line Flaw 3 from the CCSS Pipe Side at $800 \mathrm{kHz}$ and an Axial Position of $-130 \mathrm{~mm}$. The upper portion of the flaw is boxed and isolated in the C-scan, top view. 


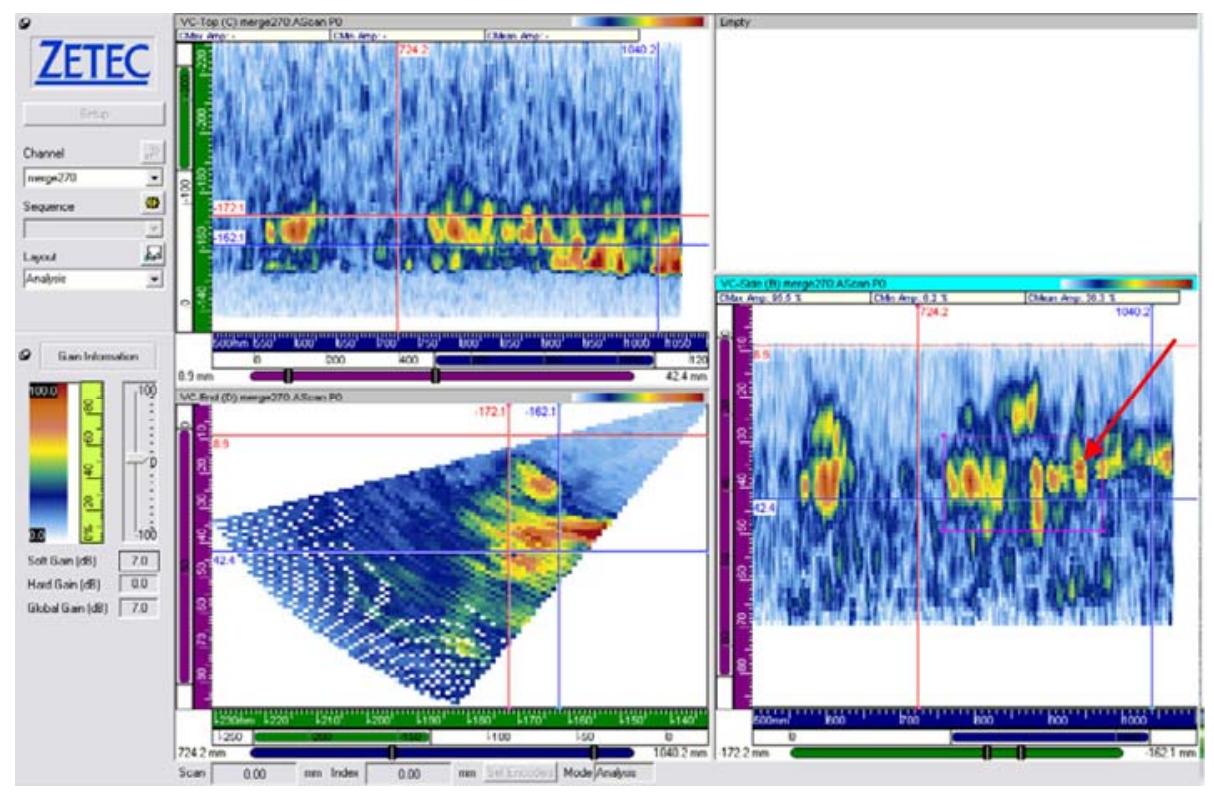

Figure A.7. Surge Line Flaw 4 from the CCSS Pipe Side at $800 \mathrm{kHz}$ and an Axial Position of -130 mm

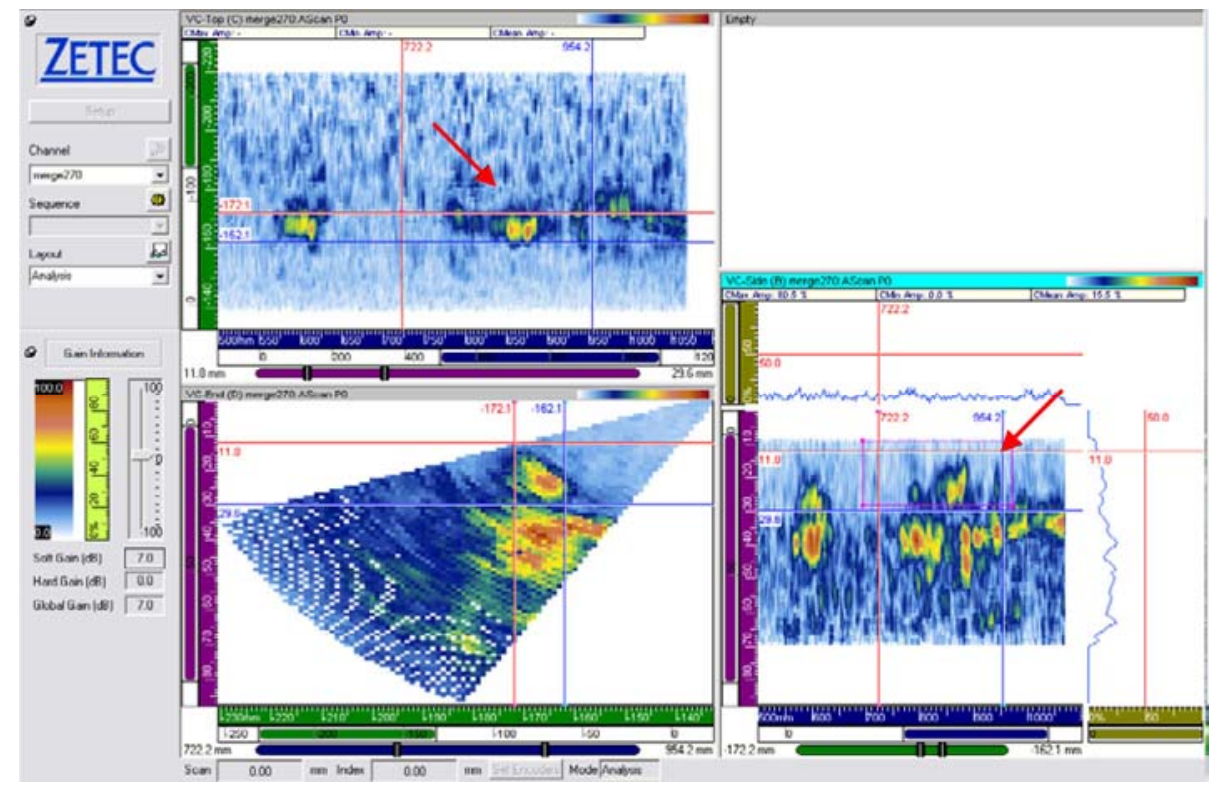

Figure A.8. Surge Line Flaw 4 from the CCSS Pipe Side at $800 \mathrm{kHz}$ and an Axial Position of $-130 \mathrm{~mm}$. The upper portion of the flaw is boxed and isolated in the C-scan, top view. 


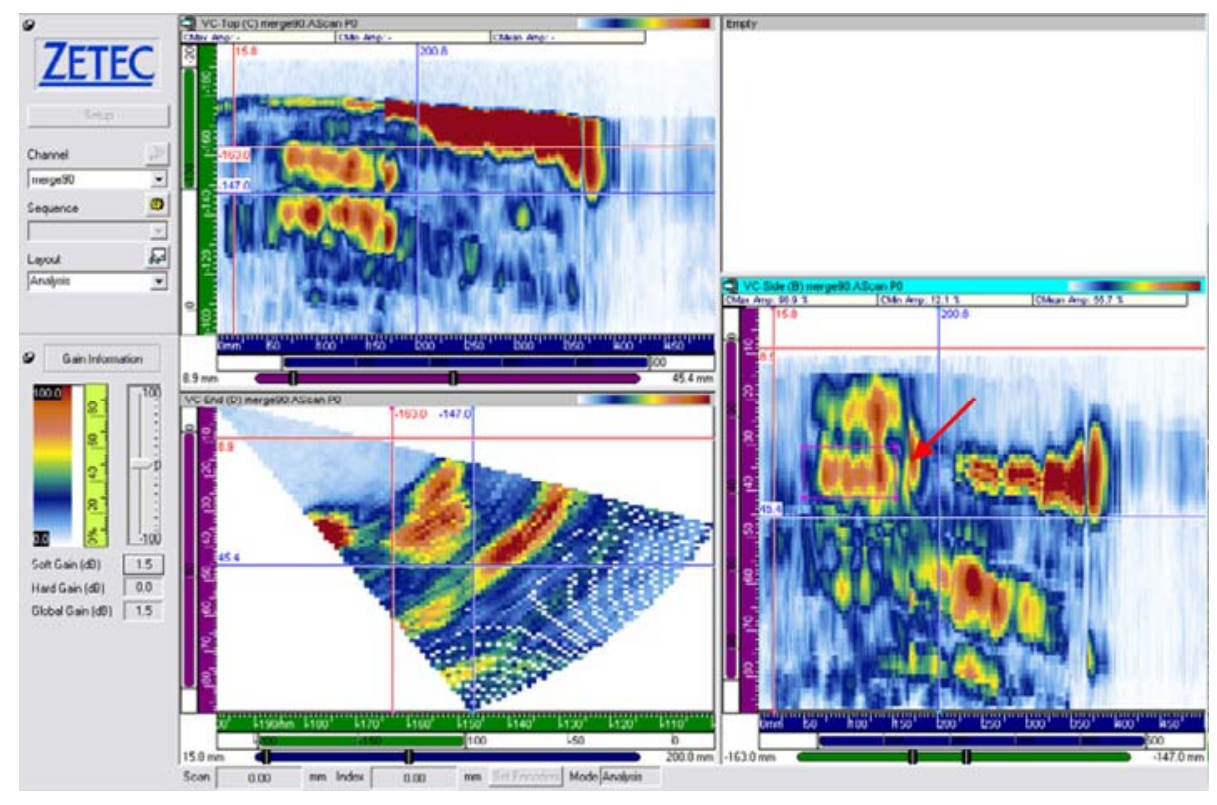

Figure A.9. Surge Line Flaw 1 from the SCSS Pipe Side at $800 \mathrm{kHz}$ and an Axial Position of $-200 \mathrm{~mm}$

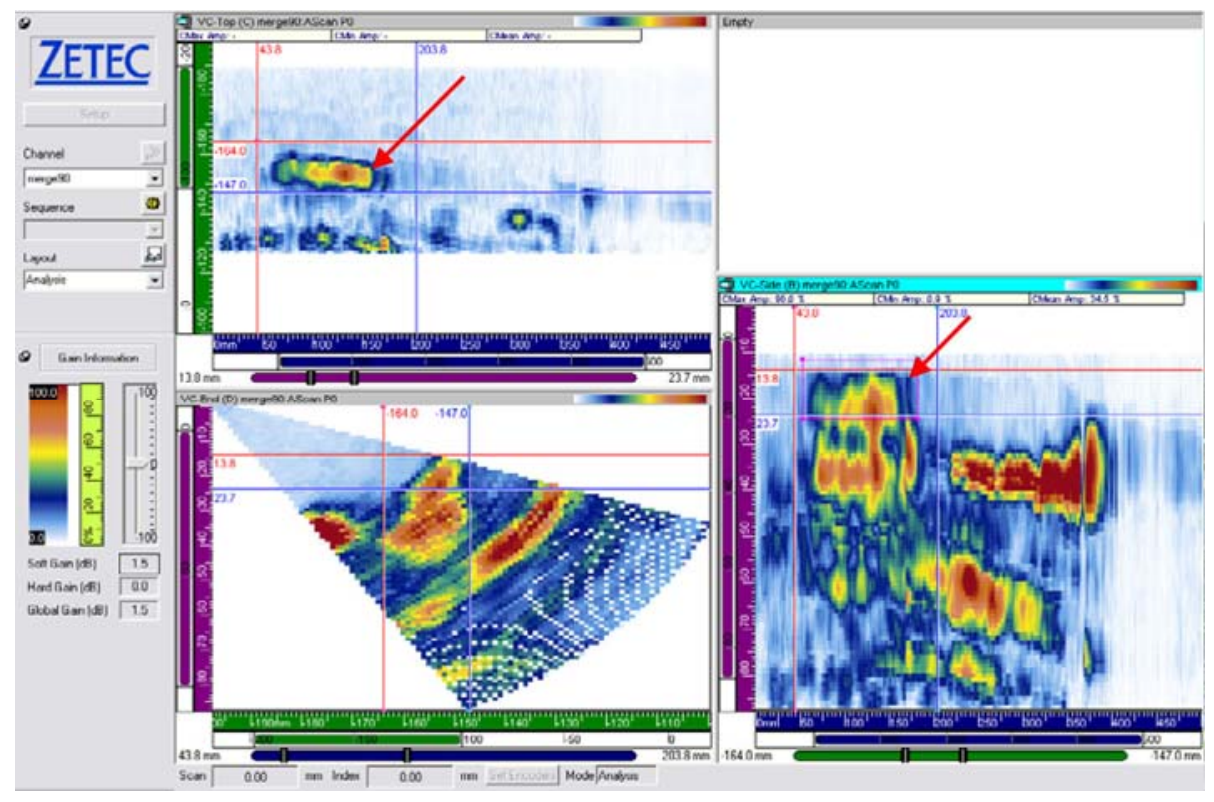

Figure A.10. Surge Line Flaw 1 from the SCSS Pipe Side at $800 \mathrm{kHz}$ and an Axial Position of -200 mm. The upper portion of the flaw is boxed and isolated in the C-scan, top view. 


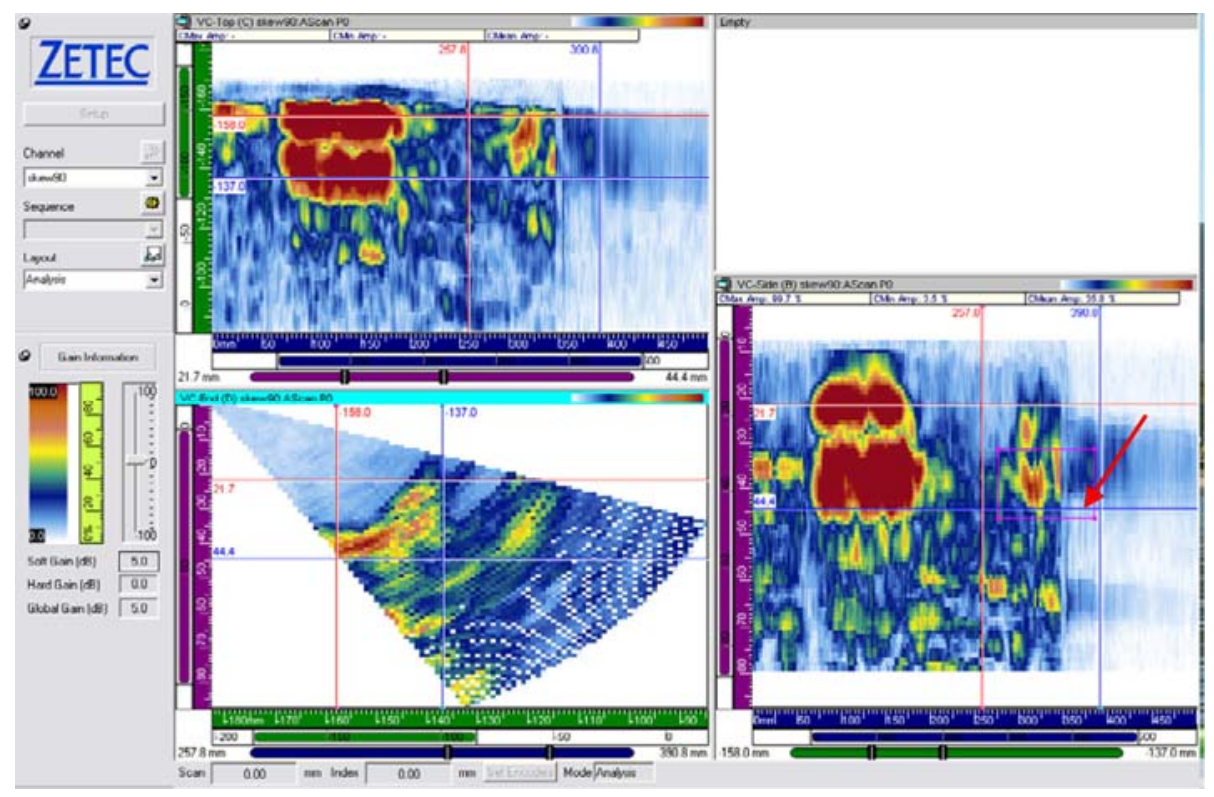

Figure A.11. Surge Line Flaw 2 from the SCSS Pipe Side at $800 \mathrm{kHz}$ and an Axial Position of - $185 \mathrm{~mm}$

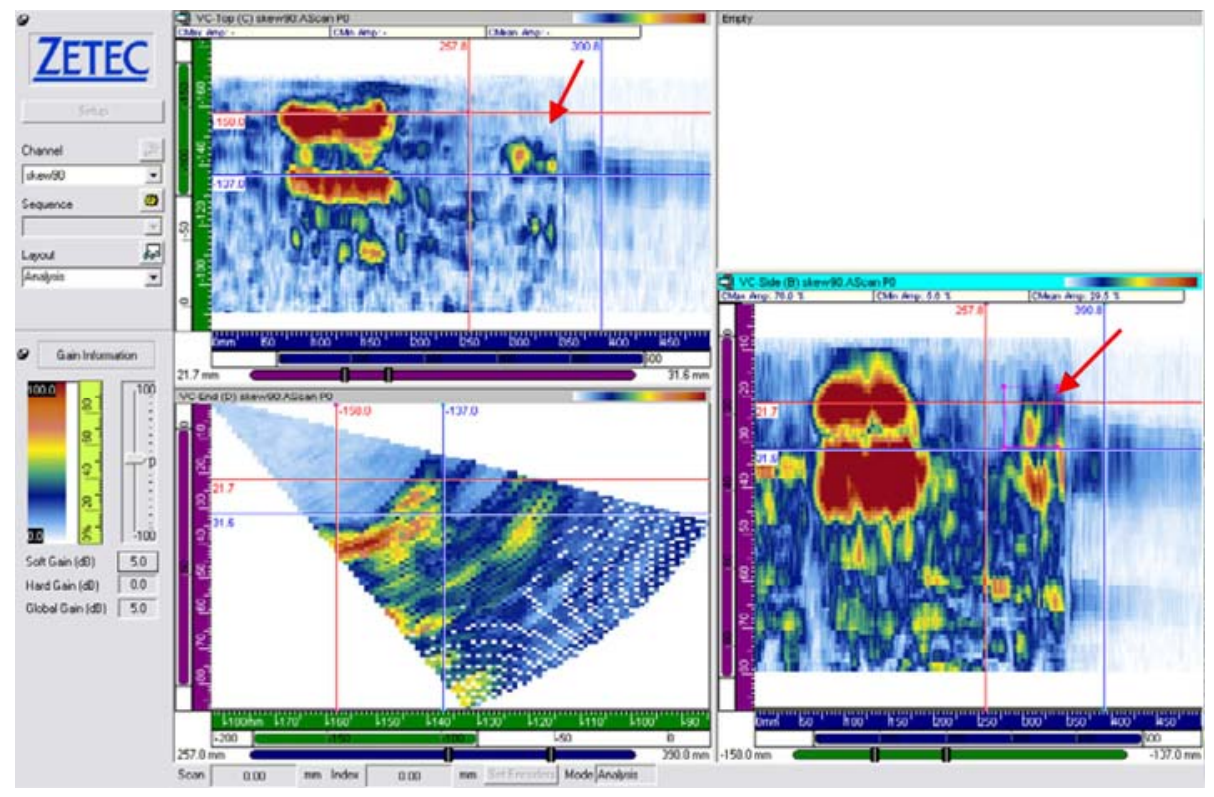

Figure A.12. Surge Line Flaw 2 from the SCSS Pipe Side at $800 \mathrm{kHz}$ and an Axial Position of $-185 \mathrm{~mm}$. The upper portion of the flaw is boxed and isolated in the C-scan, top view. 


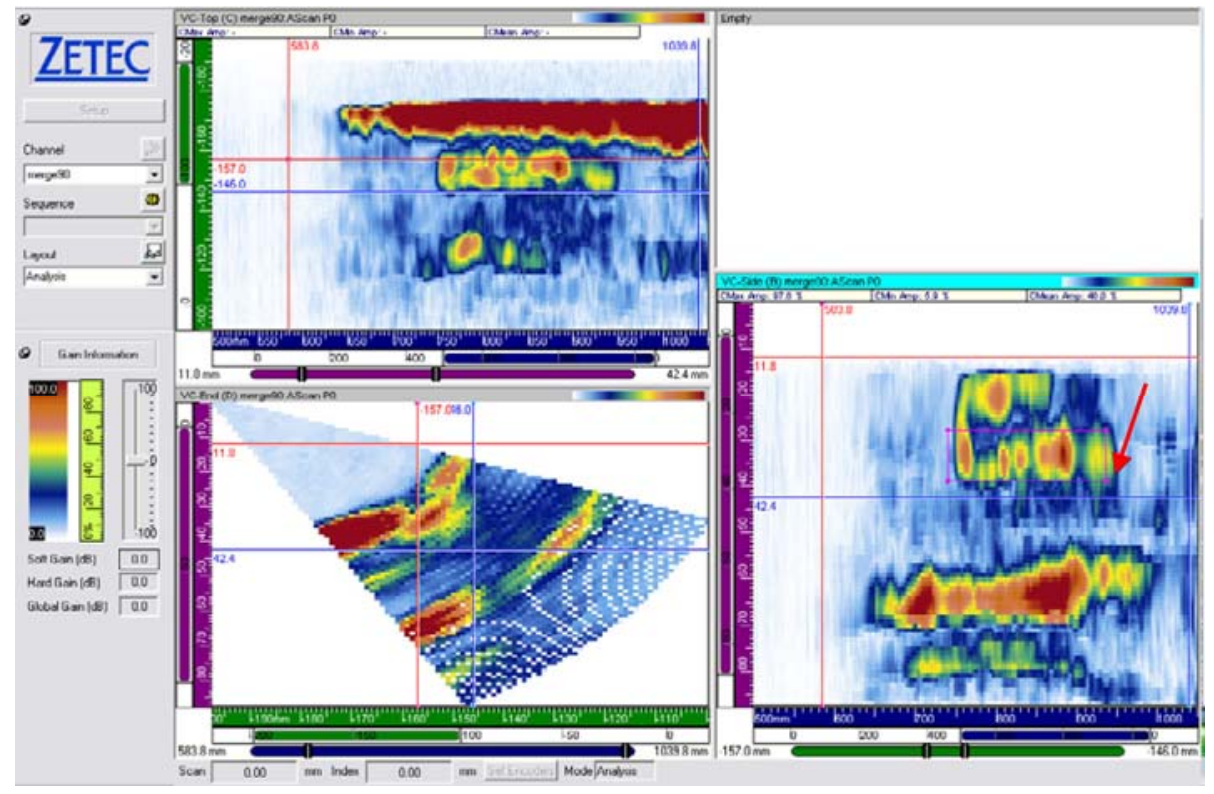

Figure A.13. Surge Line Flaw 4 from the SCSS Pipe Side at $800 \mathrm{kHz}$ and an Axial Position of $-200 \mathrm{~mm}$

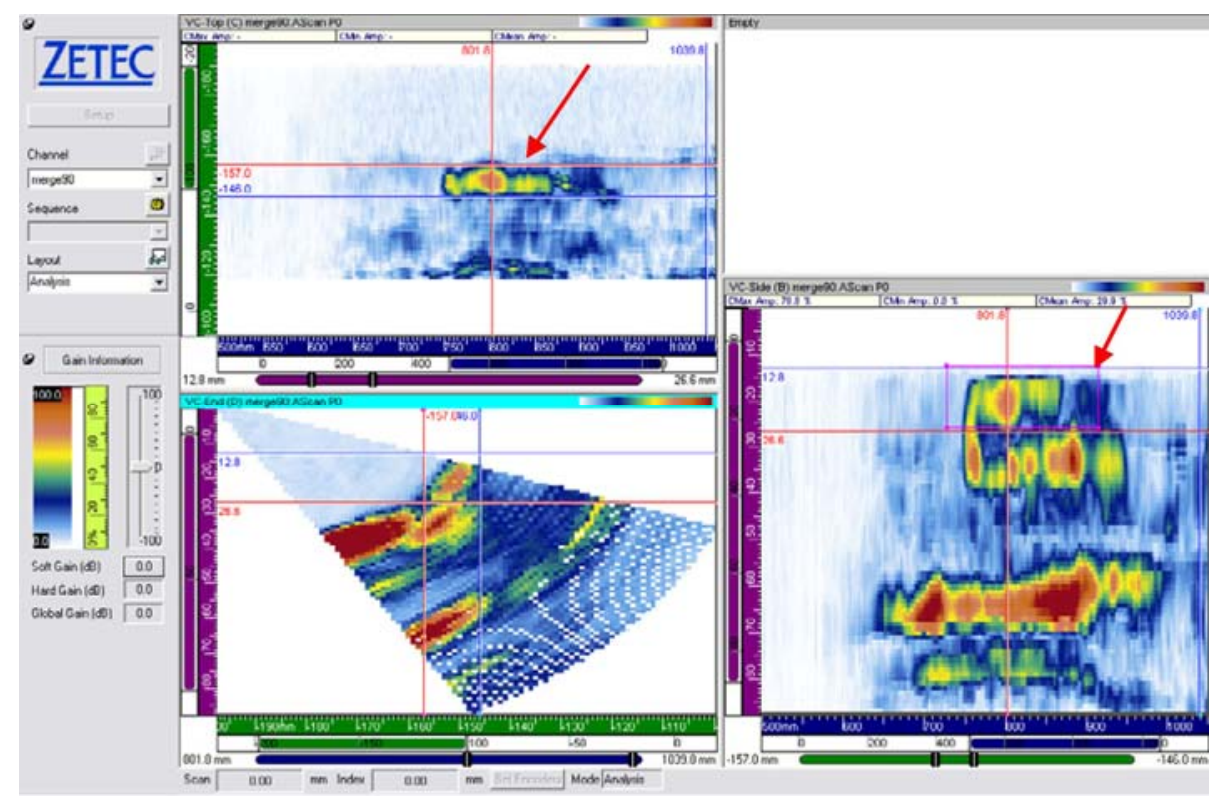

Figure A.14. Surge Line Flaw 4 from the SCSS Pipe Side at $800 \mathrm{kHz}$ and an Axial Position of $-200 \mathrm{~mm}$. The upper portion of the flaw is boxed and isolated in the C-scan, top view. 

Appendix B

1.5-MHz Phased Array Data on the Pressurizer Surge Line 



\section{Appendix B}

\section{5-MHz Phased Array Data on the Pressurizer Surge Line}

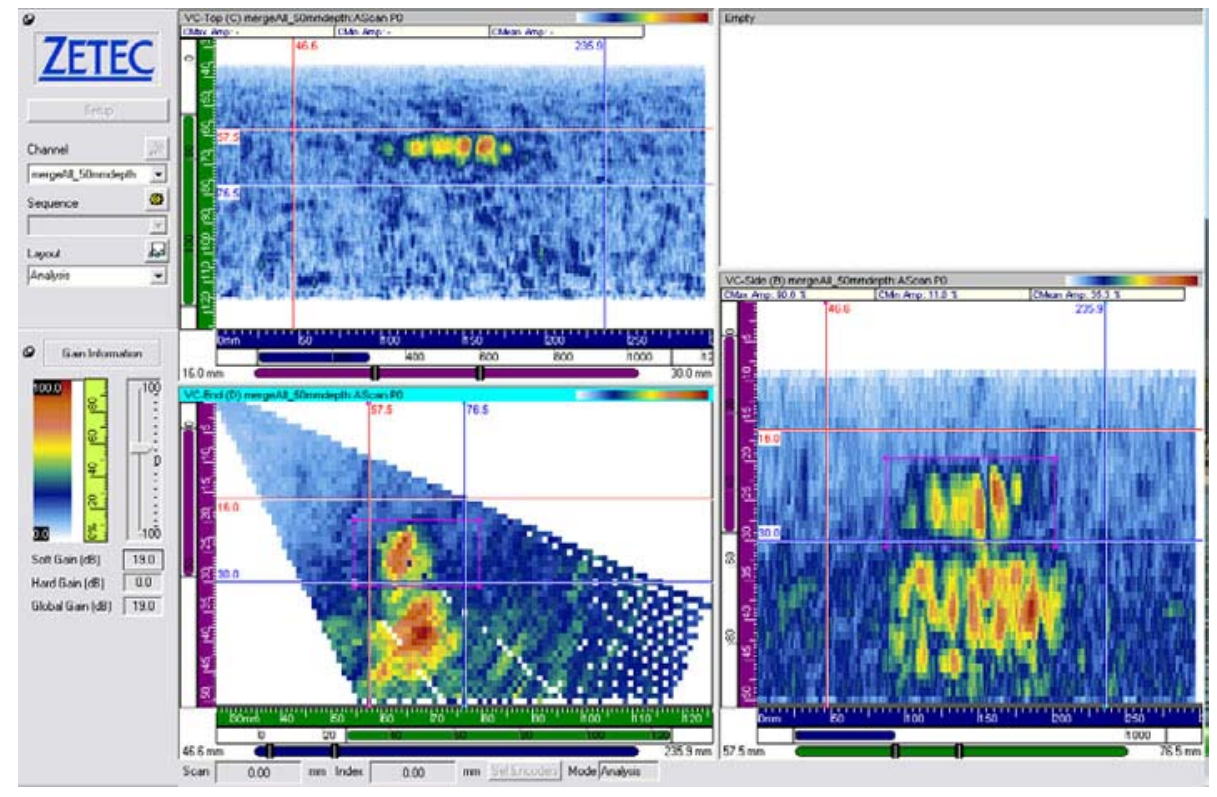

Figure B.1. Surge Line Flaw 1 from the CCSS Pipe Side at $1.5 \mathrm{MHz}$ and an Axial Position of $35 \mathrm{~mm}$

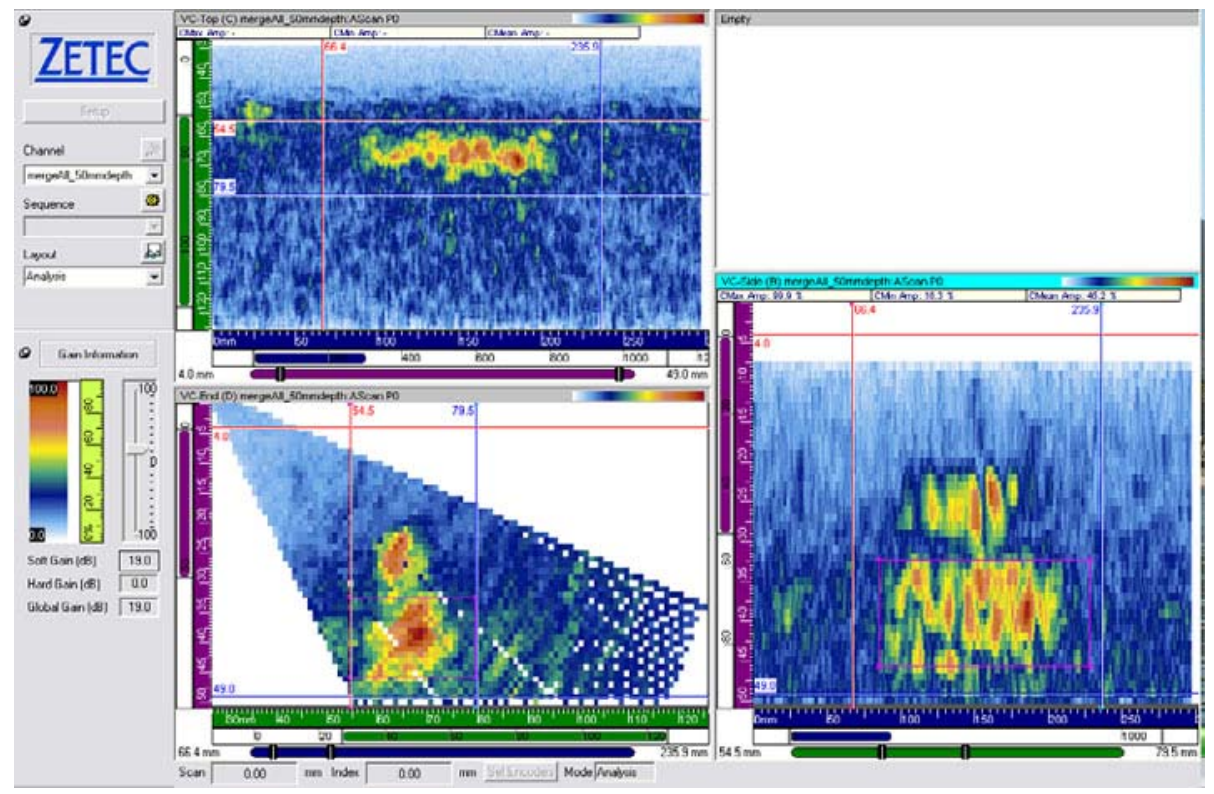

Figure B.2. Surge Line Flaw 1 from the CCSS Pipe Side at $1.5 \mathrm{MHz}$ and an Axial Position of $35 \mathrm{~mm}$. The upper portion of the flaw is boxed and isolated in the C-scan, top view. 


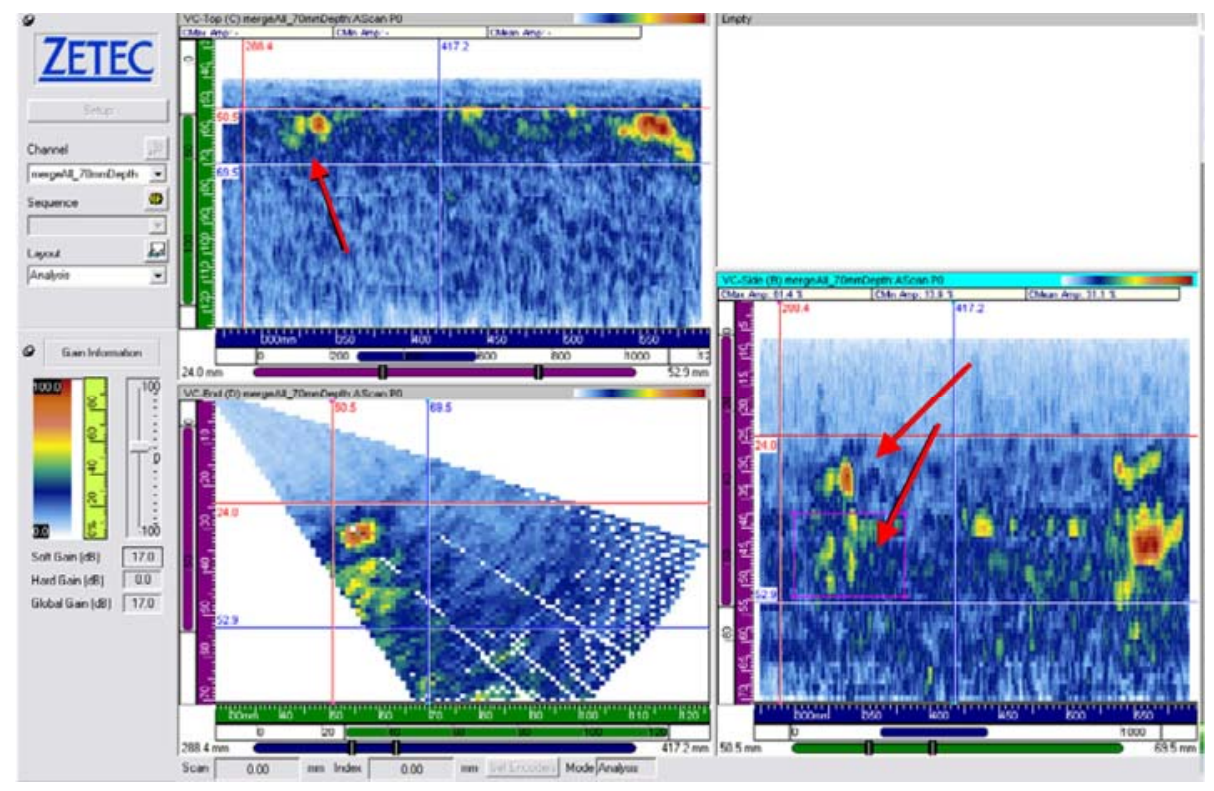

Figure B.3. Surge Line Flaw 2 from the CCSS Pipe Side at $1.5 \mathrm{MHz}$ and an Axial Position of $35 \mathrm{~mm}$

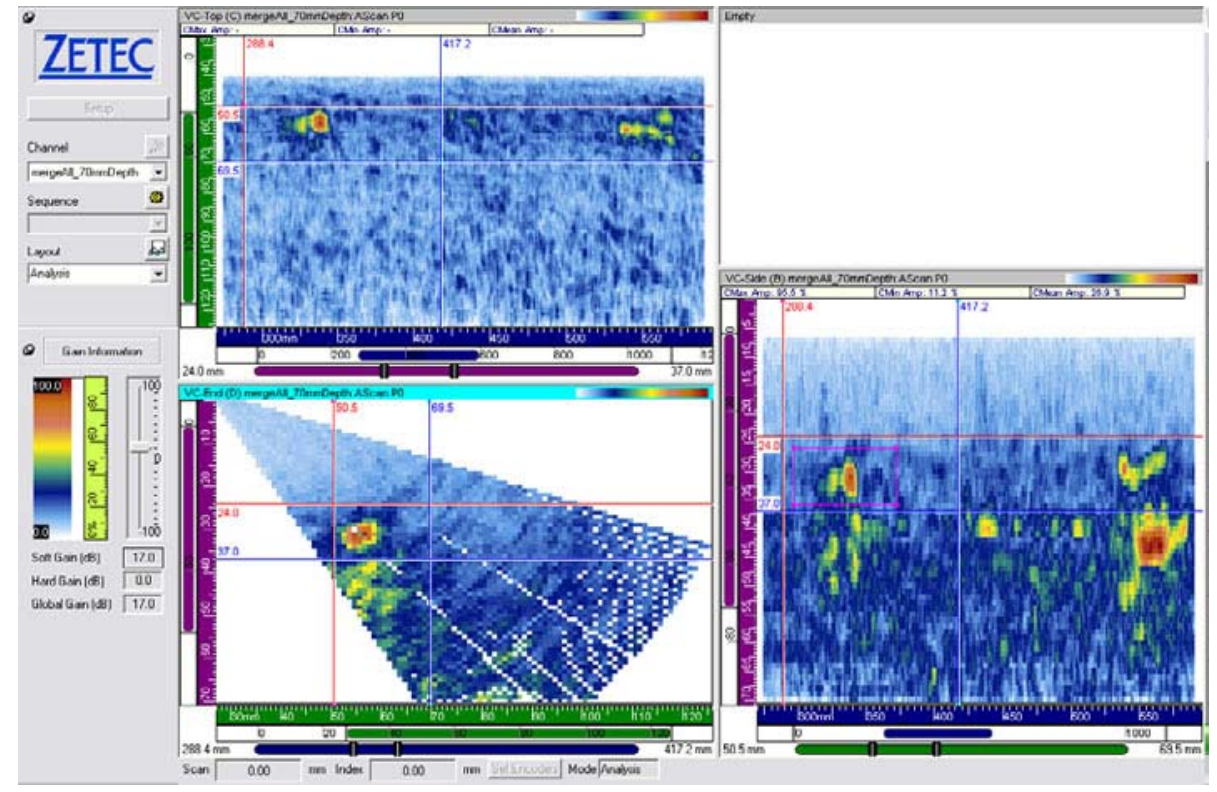

Figure B.4. Surge Line Flaw 2 from the CCSS Pipe Side at $1.5 \mathrm{MHz}$ and an Axial Position of $35 \mathrm{~mm}$. The upper portion of the flaw is boxed and isolated in the C-scan, top view. 


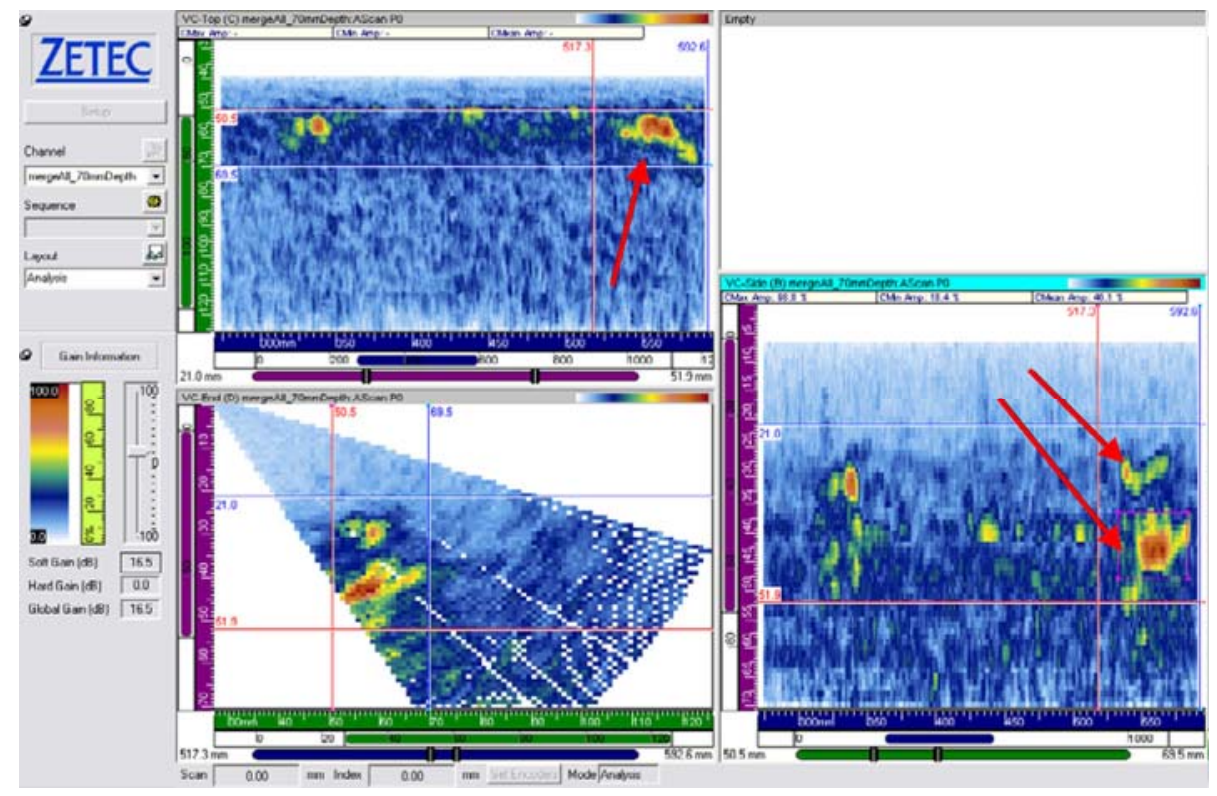

Figure B.5. Surge Line Flaw 3 from the CCSS Pipe Side at $1.5 \mathrm{MHz}$ and an Axial Position of $35 \mathrm{~mm}$

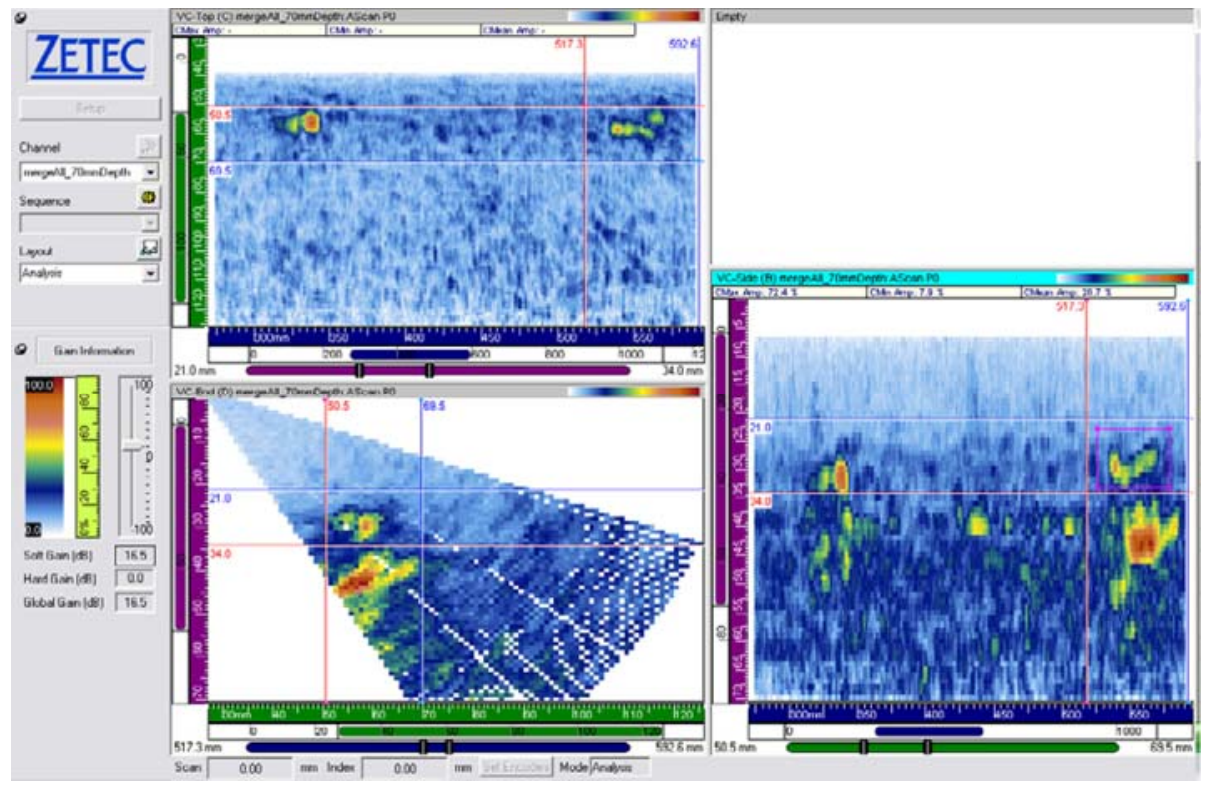

Figure B.6. Surge Line Flaw 3 from the CCSS Pipe Side at 1.5 MHz and an Axial Position of $35 \mathrm{~mm}$. The upper portion of the flaw is boxed and isolated in the C-scan, top view. 


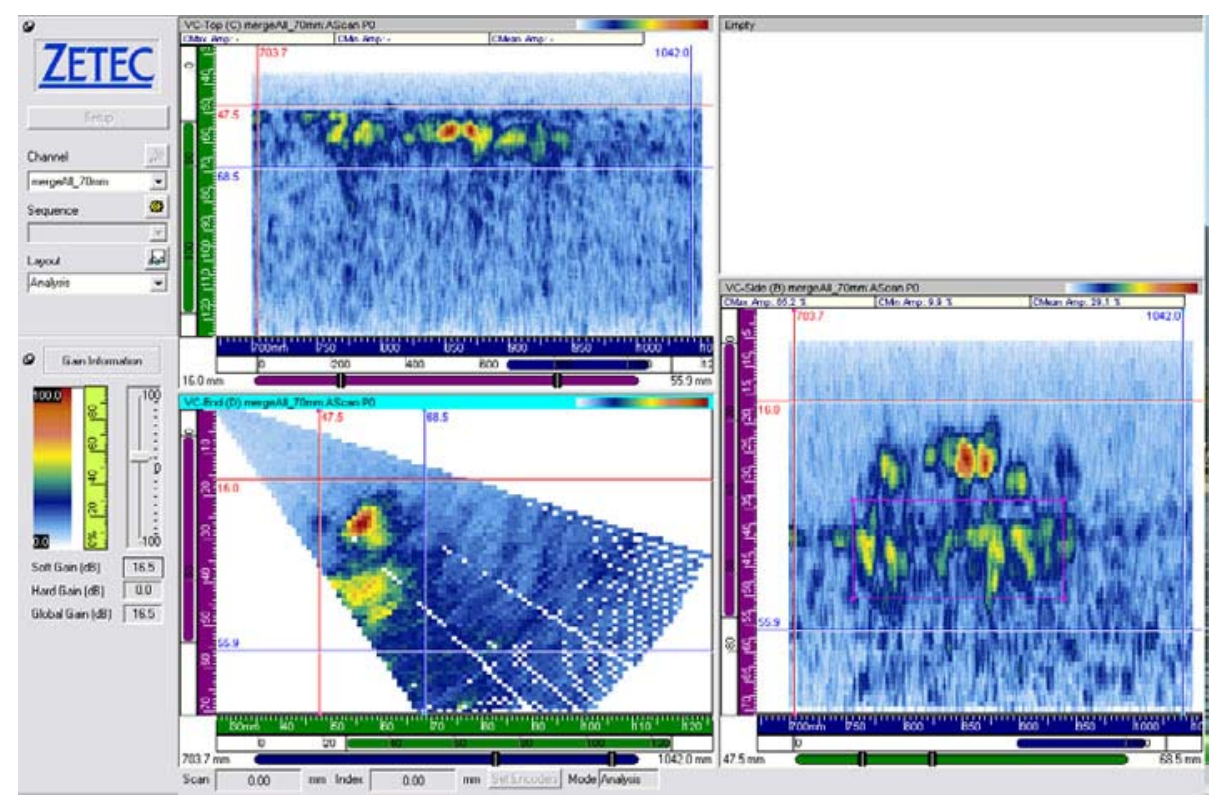

Figure B.7. Surge Line Flaw 4 from the CCSS Pipe Side at $1.5 \mathrm{MHz}$ and an Axial Position of $35 \mathrm{~mm}$

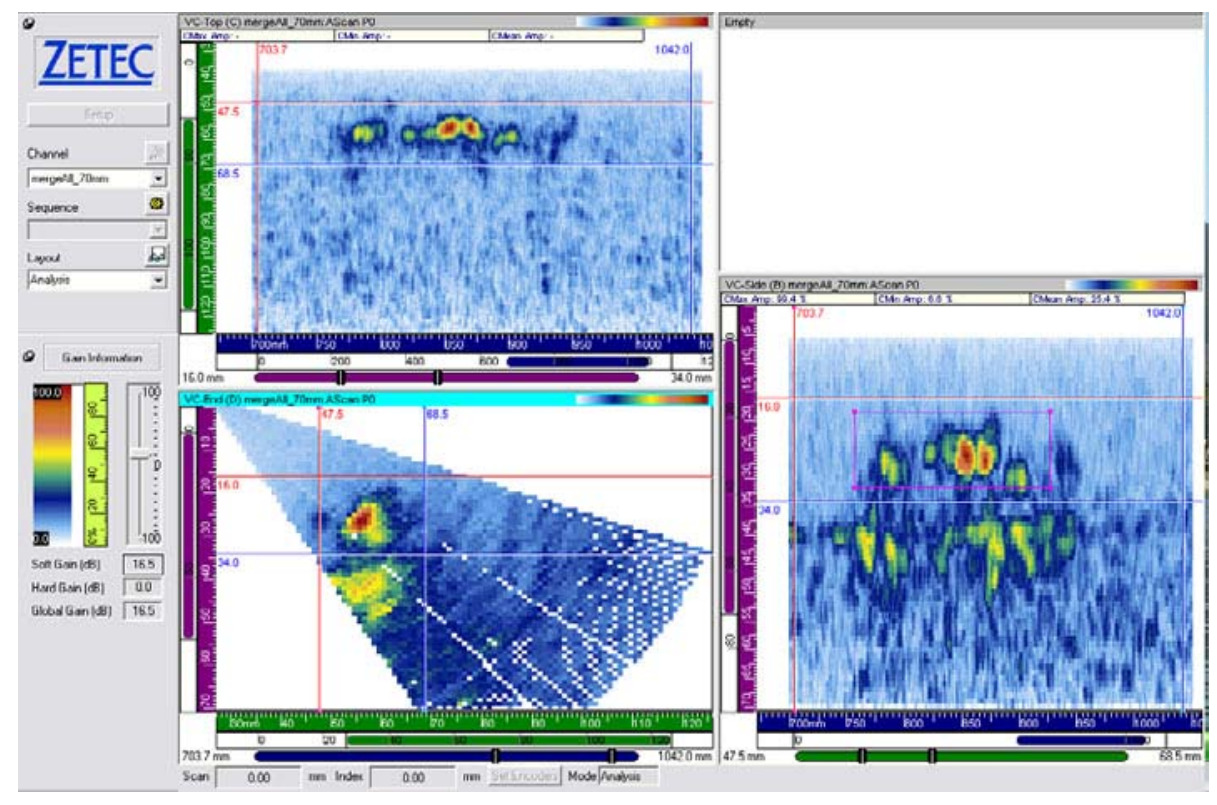

Figure B.8. Surge Line Flaw 4 from the CCSS Pipe Side at 1.5 MHz and an Axial Position of $35 \mathrm{~mm}$. The upper portion of the flaw is boxed and isolated in the C-scan, top view. 


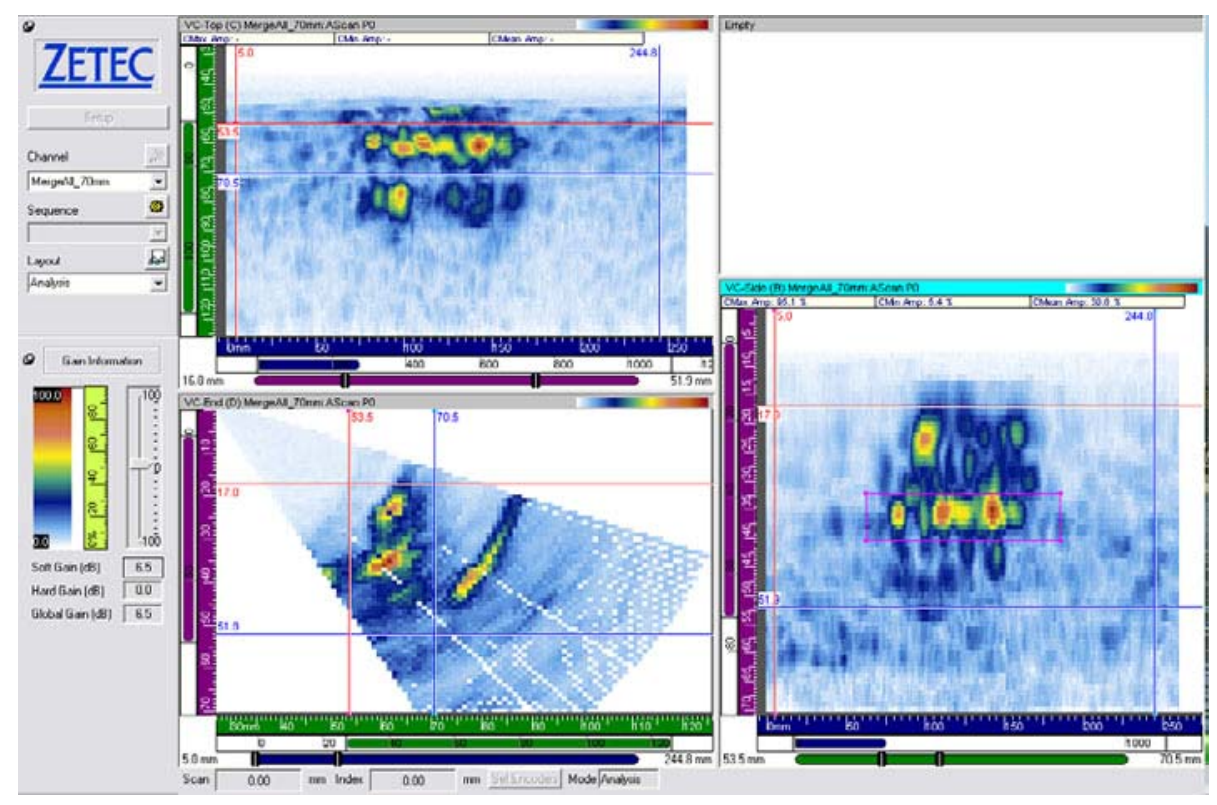

Figure B.9. Surge Line Flaw 1 from the SCSS Pipe Side at $1.5 \mathrm{MHz}$ and an Axial Position of $25 \mathrm{~mm}$

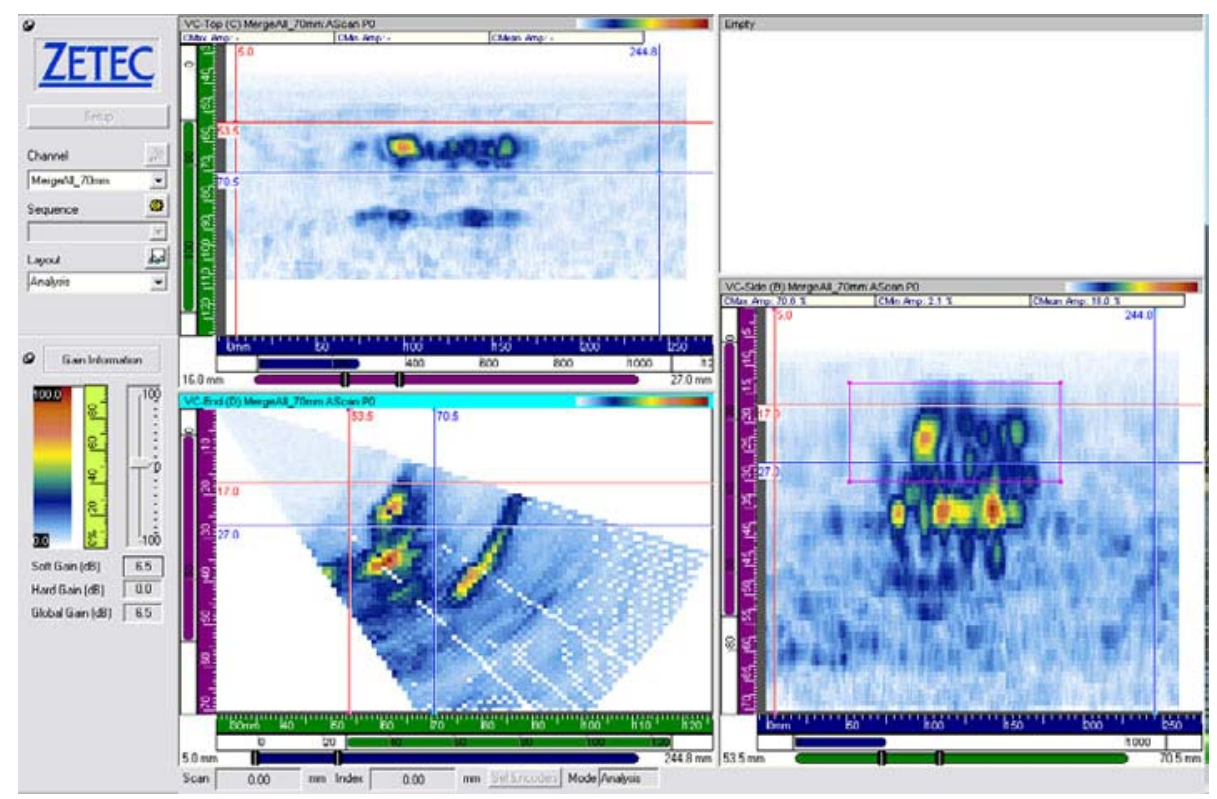

Figure B.10. Surge Line Flaw 1 from the SCSS Pipe Side at $1.5 \mathrm{MHz}$ and an Axial Position of $25 \mathrm{~mm}$. The upper portion of the flaw is boxed and isolated in the C-scan, top view. 


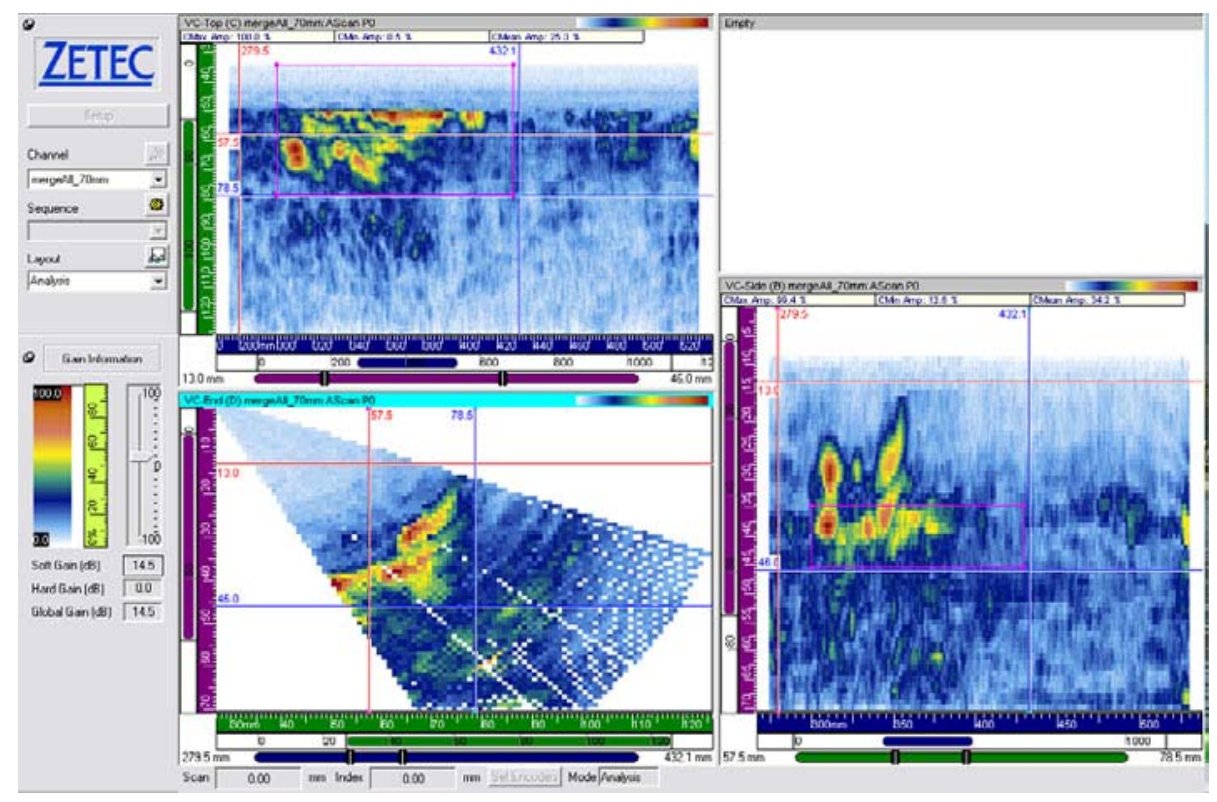

Figure B.11. Surge Line Flaw 2 from the SCSS Pipe Side at $1.5 \mathrm{MHz}$ and an Axial Position of $25 \mathrm{~mm}$

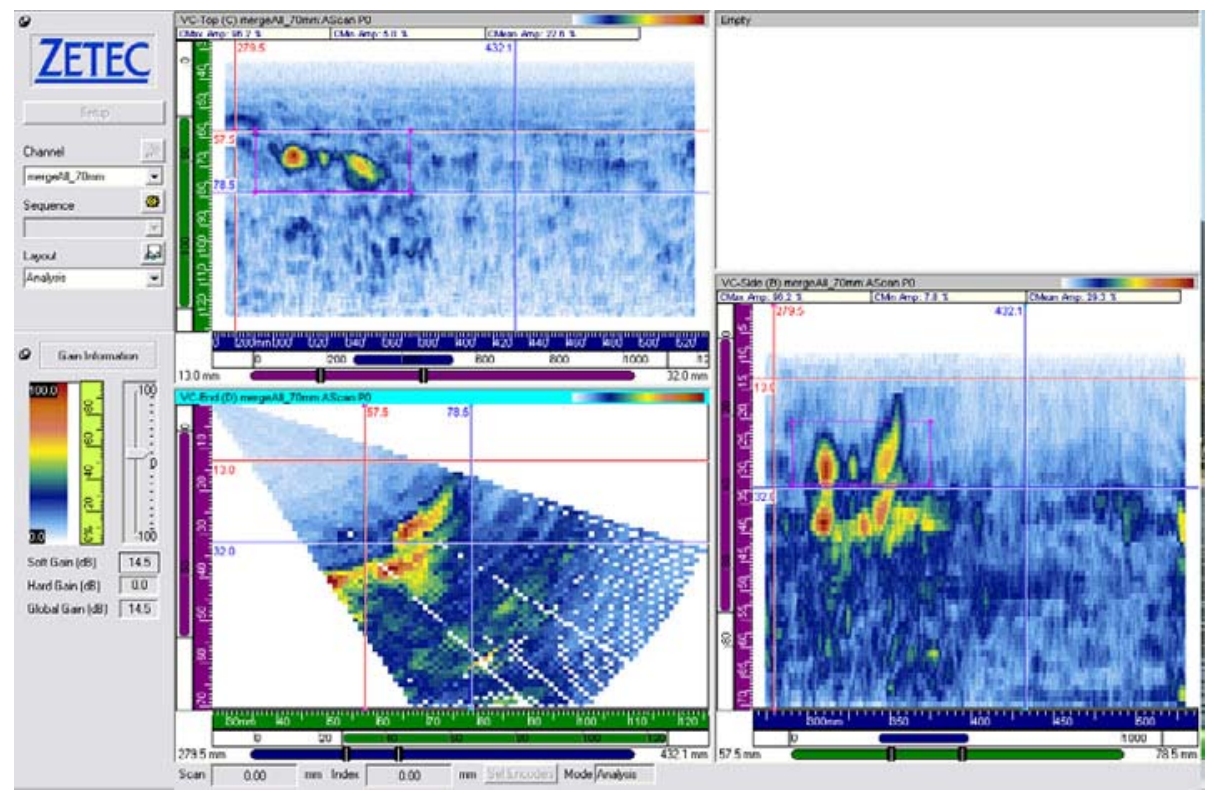

Figure B.12. Surge Line Flaw 2 from the SCSS Pipe Side at $1.5 \mathrm{MHz}$ and an Axial Position of $25 \mathrm{~mm}$. The upper portion of the flaw is boxed and isolated in the C-scan, top view. 


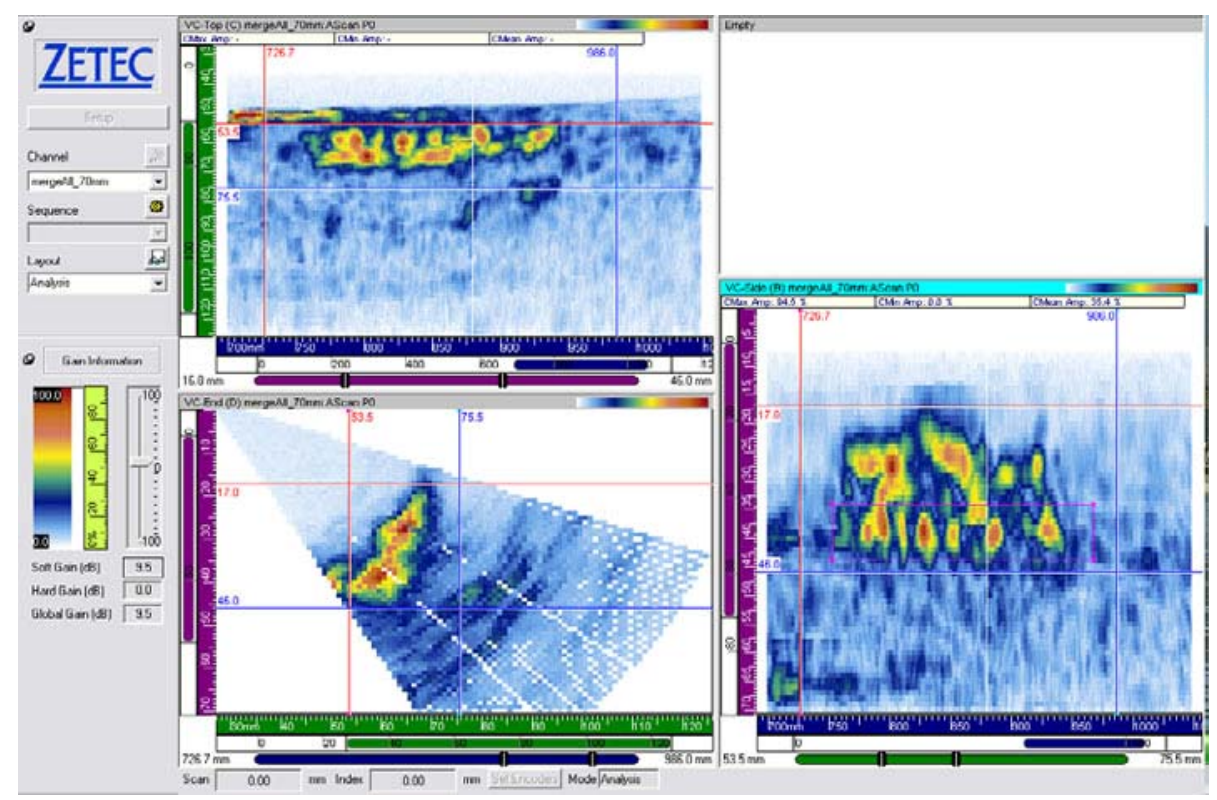

Figure B.13. Surge Line Flaw 4 from the SCSS Pipe Side at $1.5 \mathrm{MHz}$ and an Axial Position of $25 \mathrm{~mm}$

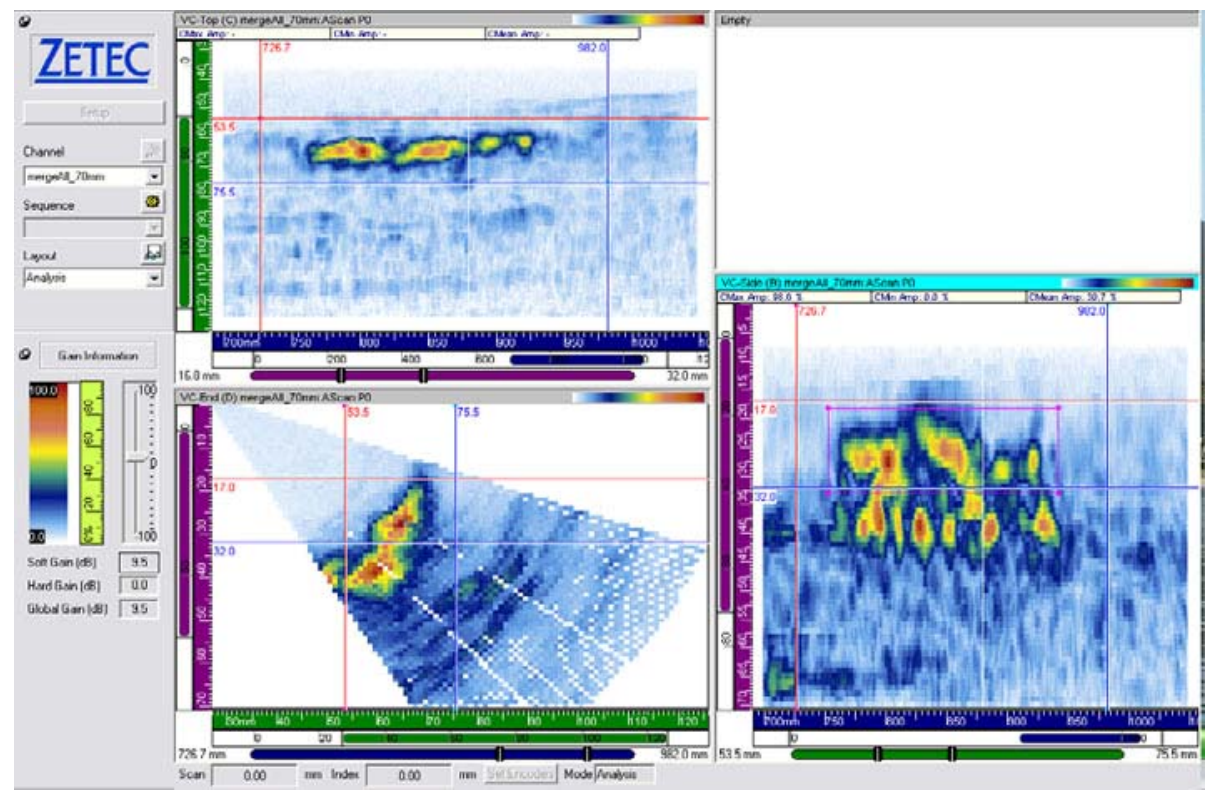

Figure B.14. Surge Line Flaw 4 from the SCSS Pipe Side at $1.5 \mathrm{MHz}$ and an Axial Position of $25 \mathrm{~mm}$. The upper portion of the flaw is boxed and isolated in the C-scan, top view. 

Appendix C

WOG Data with the 800-kHz Phased Array Probe 



\section{Appendix C}

\section{WOG Data with the 800-kHz Phased Array Probe}

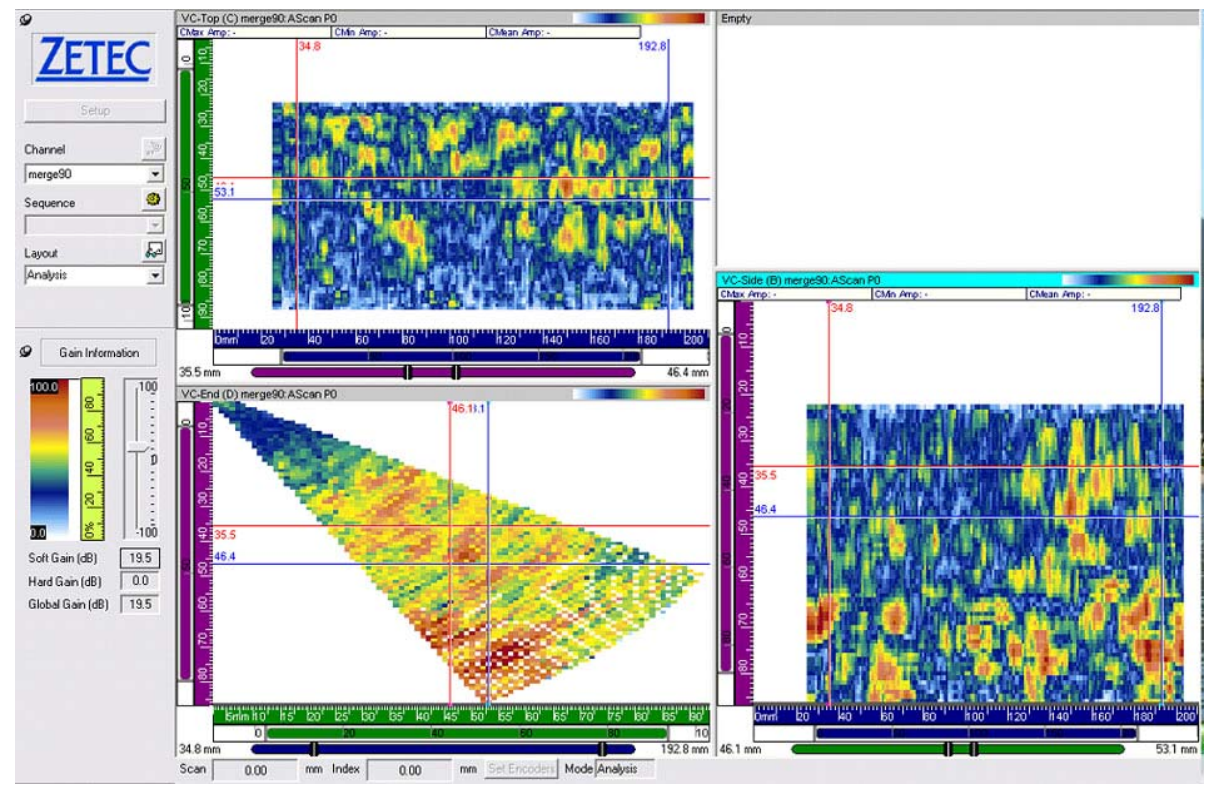

Figure C.1. $800-\mathrm{kHz}$ Data from ONP-3-5 from the CCSS Side of the Weld. The flaw was not detected.

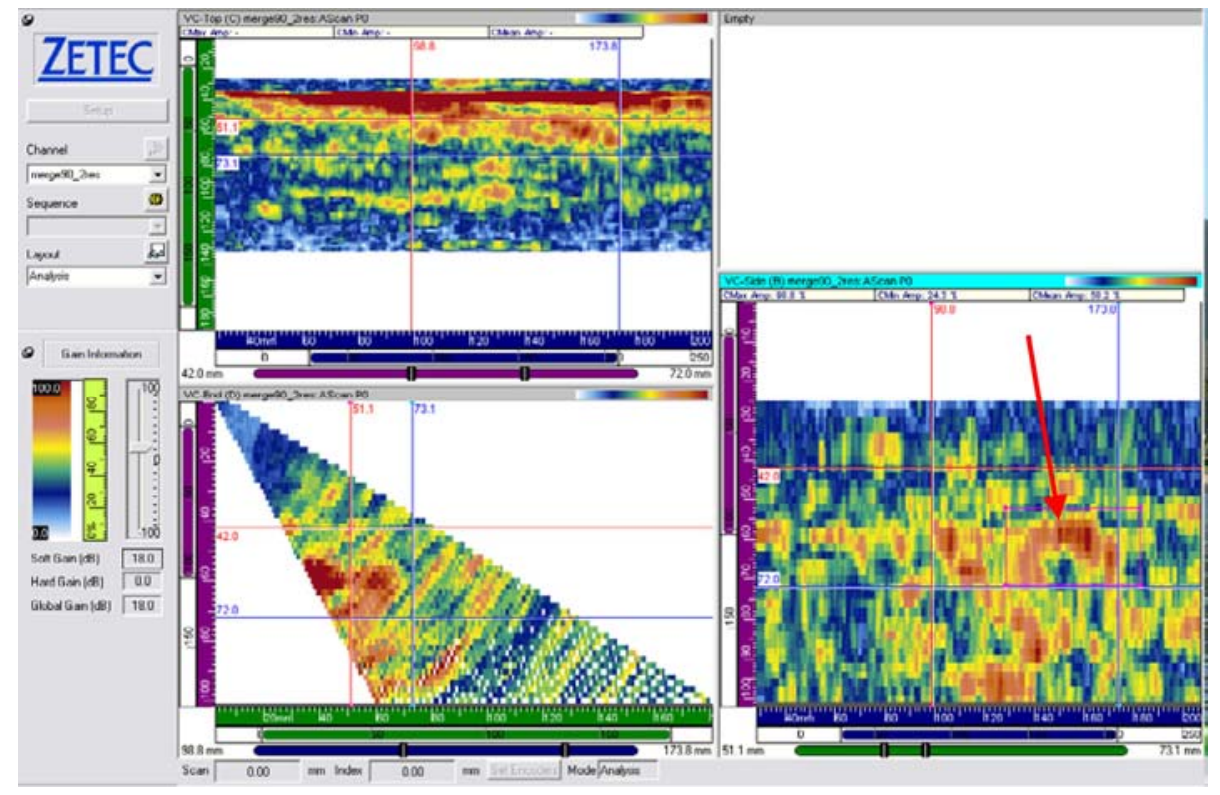

Figure C.2. $800-\mathrm{kHz}$ Data from OPE-5 from the CCSS Side of the Weld. The flaw is marginally detected. 


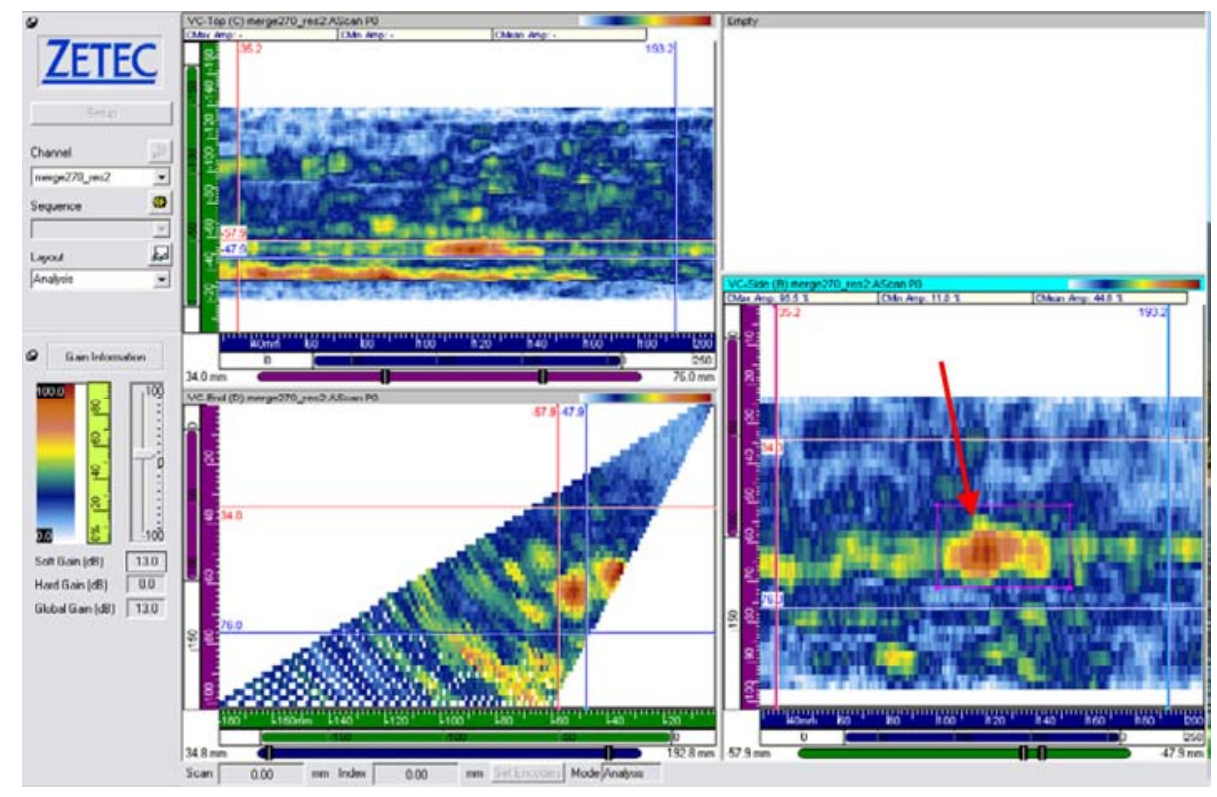

Figure C.3. $800-\mathrm{kHz}$ Data from OPE-5 from the SCSS Side of the Weld. The flaw is detected.

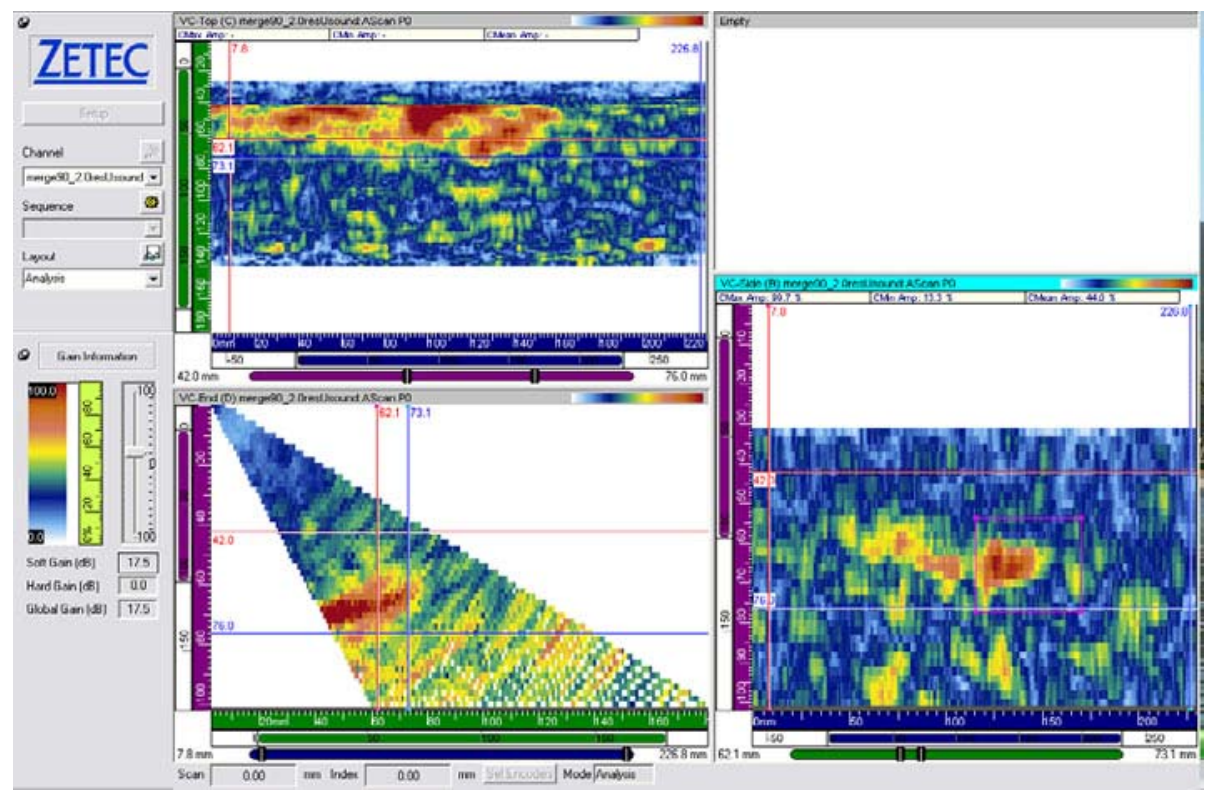

Figure C.4. $800-\mathrm{kHz}$ Data from MPE-6 from the CCSS Side of the Weld. The flaw is detected. 


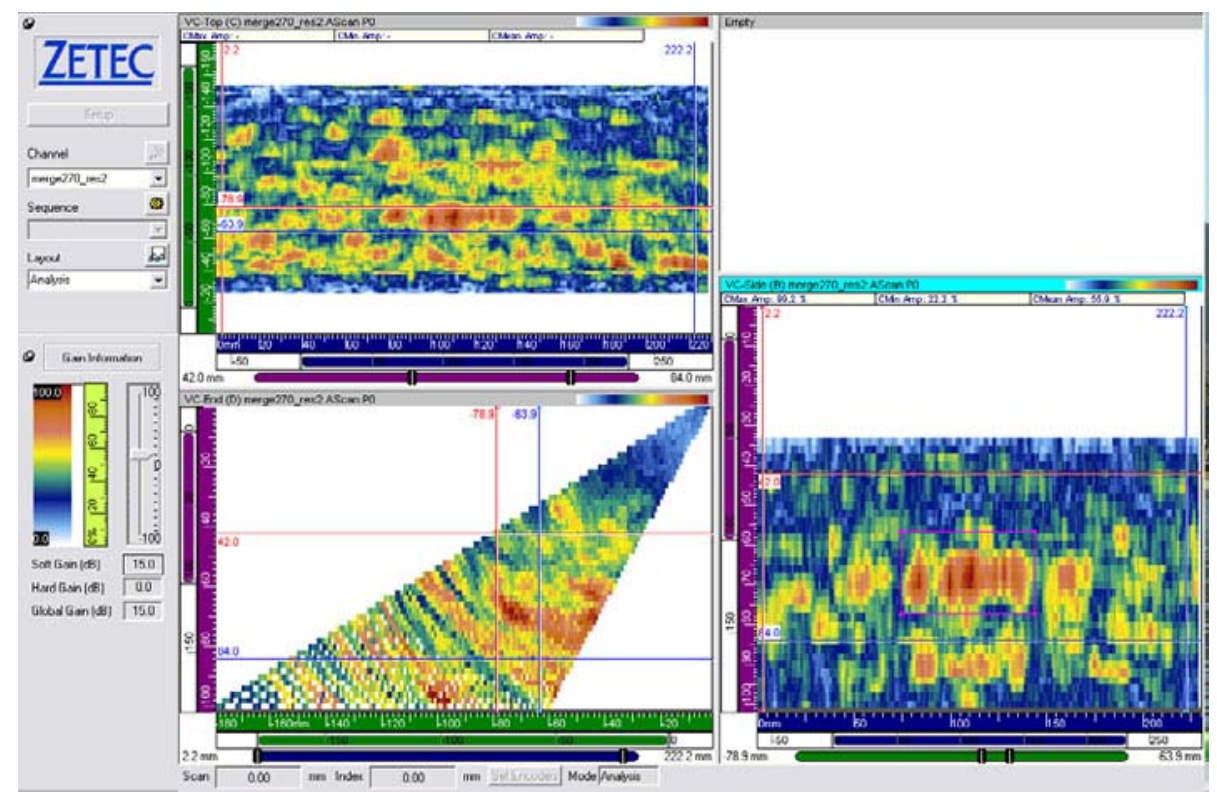

Figure C.5. $800-\mathrm{kHz}$ Data from MPE-6 from the SCSS Side of the Weld. The flaw is detected. 

Appendix D

800-kHz Phased Array Data for Delta-Technique Evaluation 



\section{Appendix D}

\section{0-kHz Phased Array Data for Delta-Technique Evaluation}

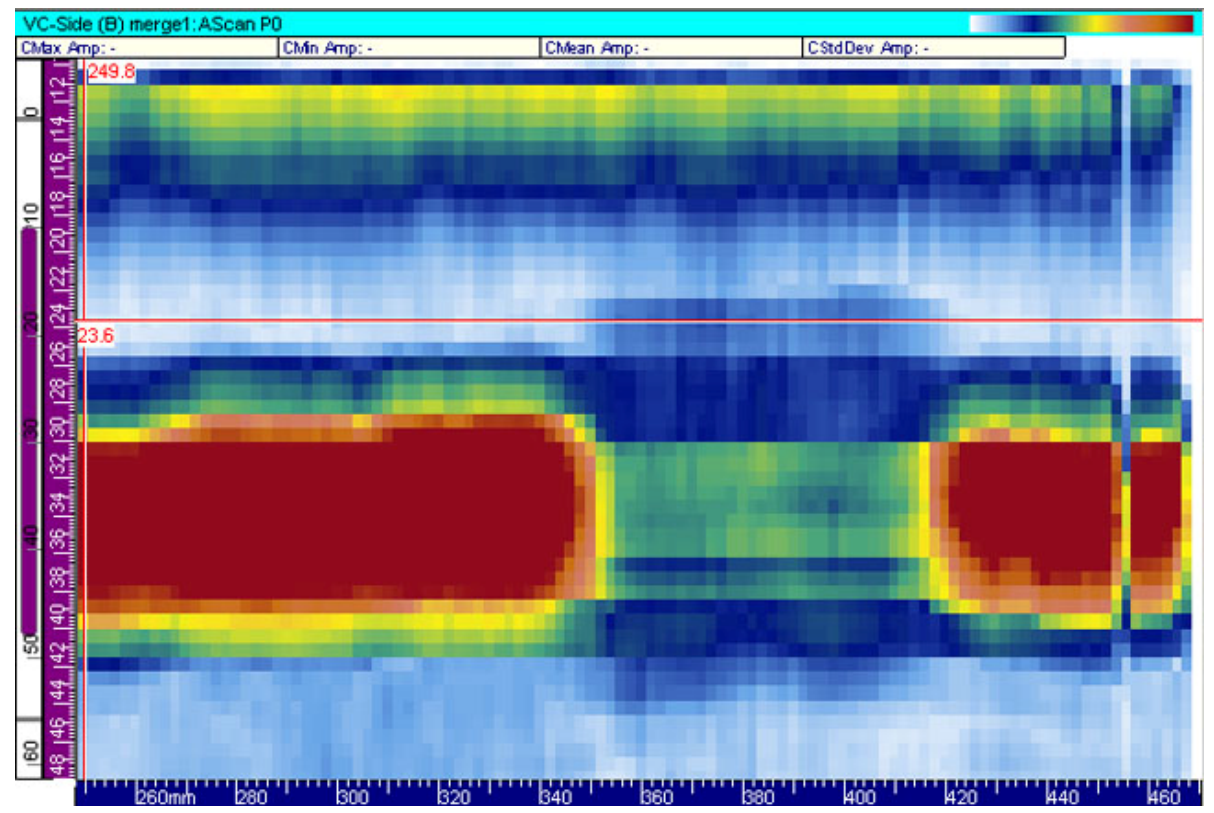

Figure D.1. Wrought Stainless, 30\% Through-Wall EDM Notch

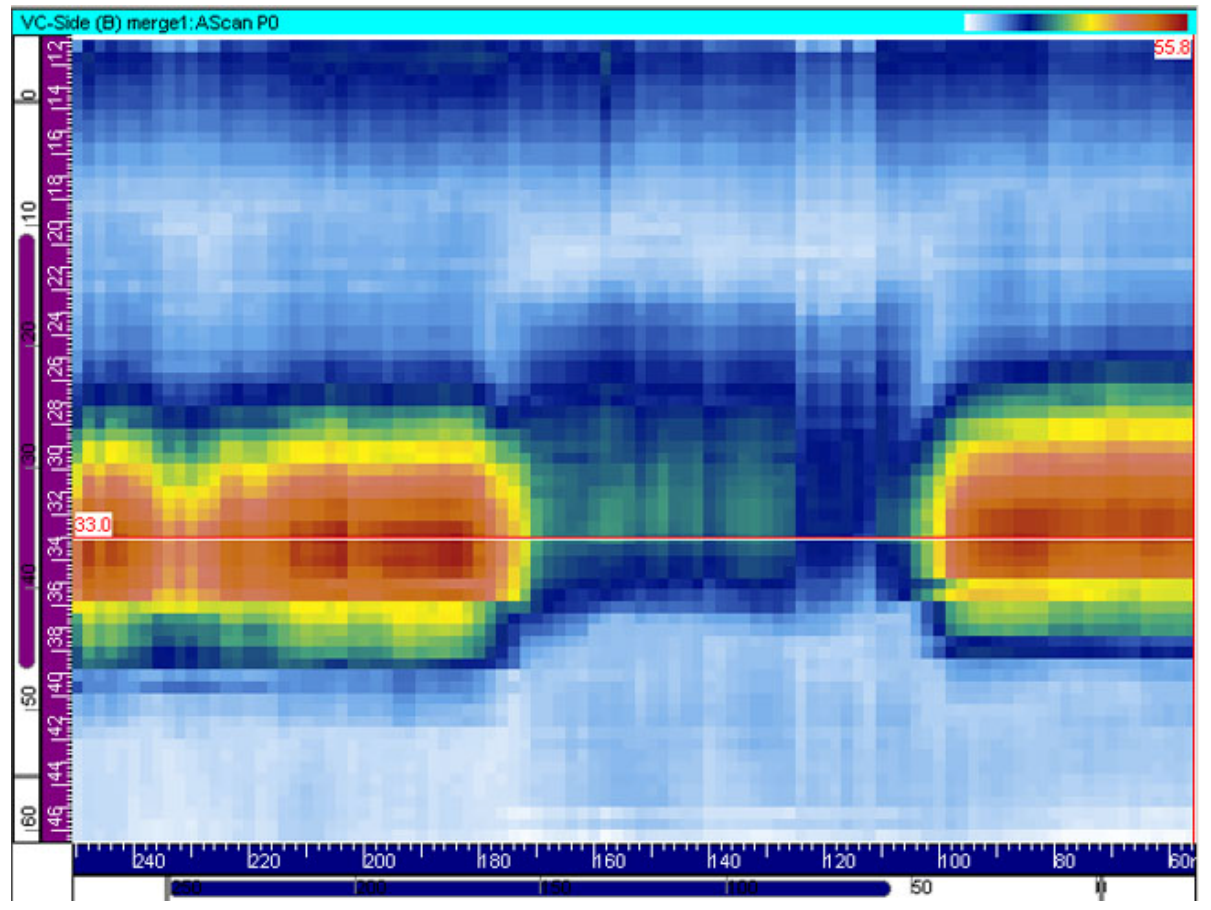

Figure D.2. Wrought Stainless, 20\% Through-Wall EDM Notch 


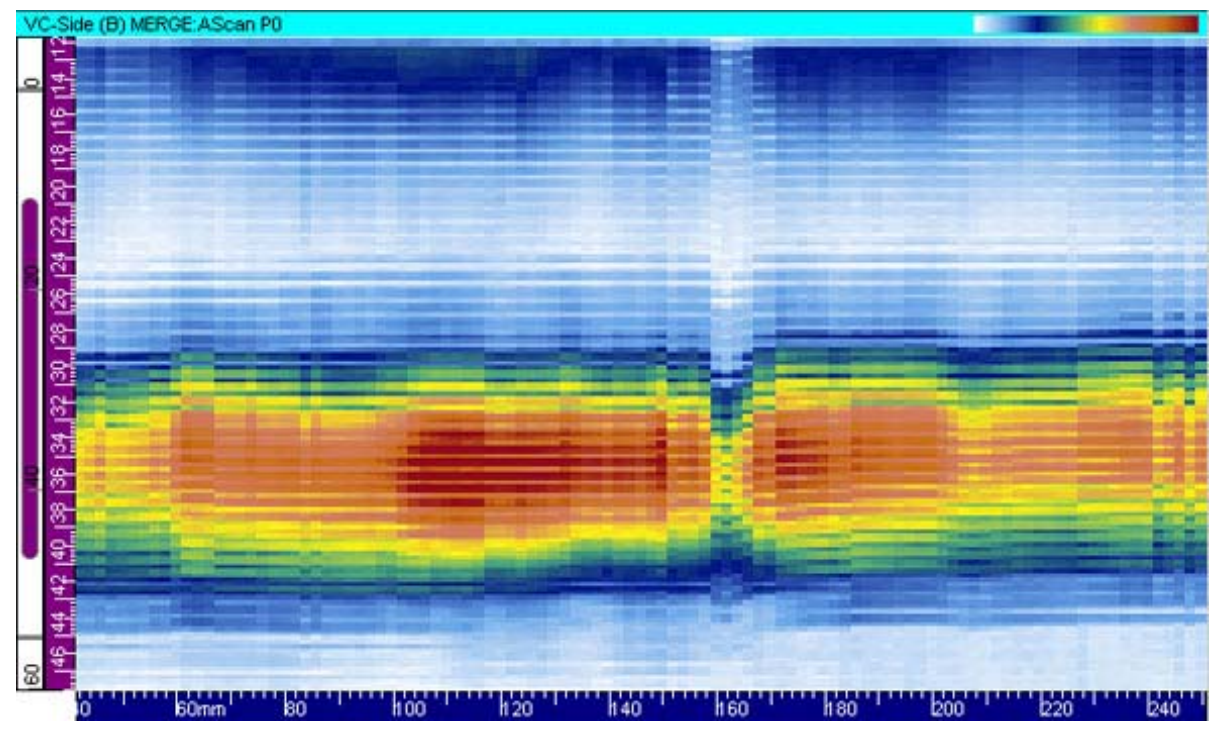

Figure D.3. Wrought Stainless, 10\% Through-Wall EDM Notch (not detected)

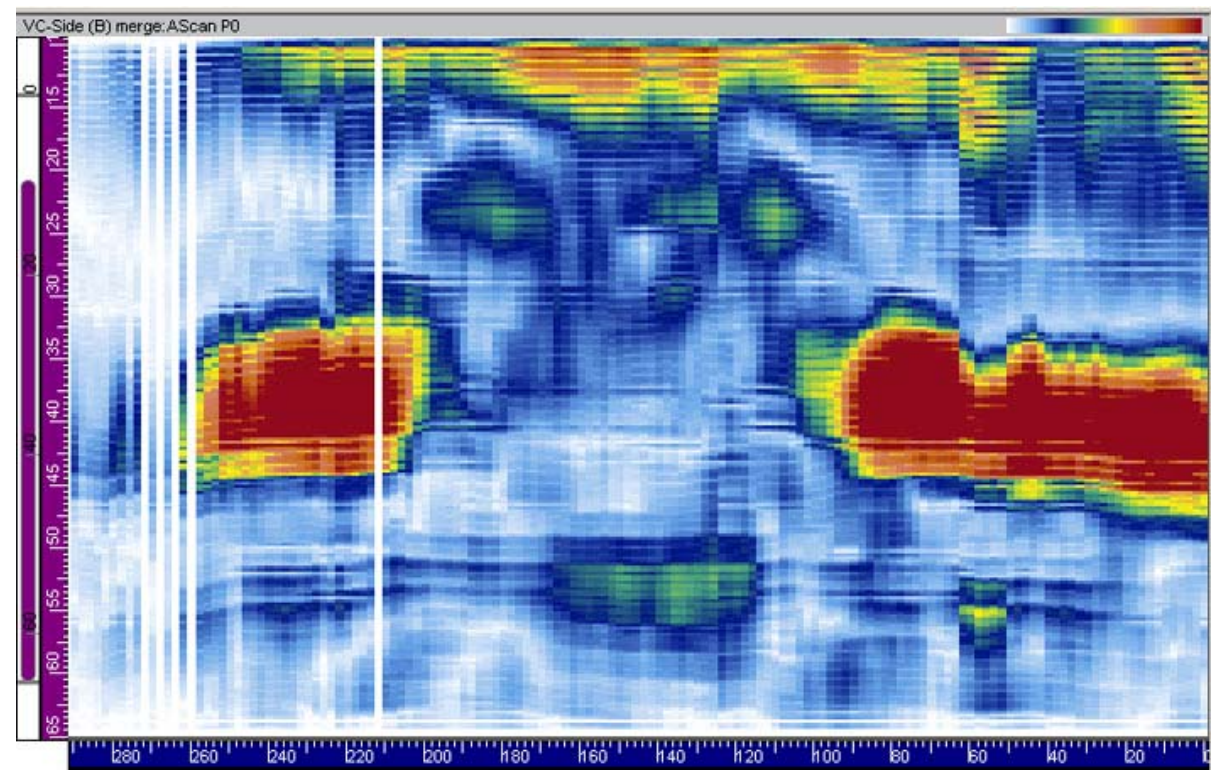

Figure D.4. Pressurizer Surge Line, Flaw 1 


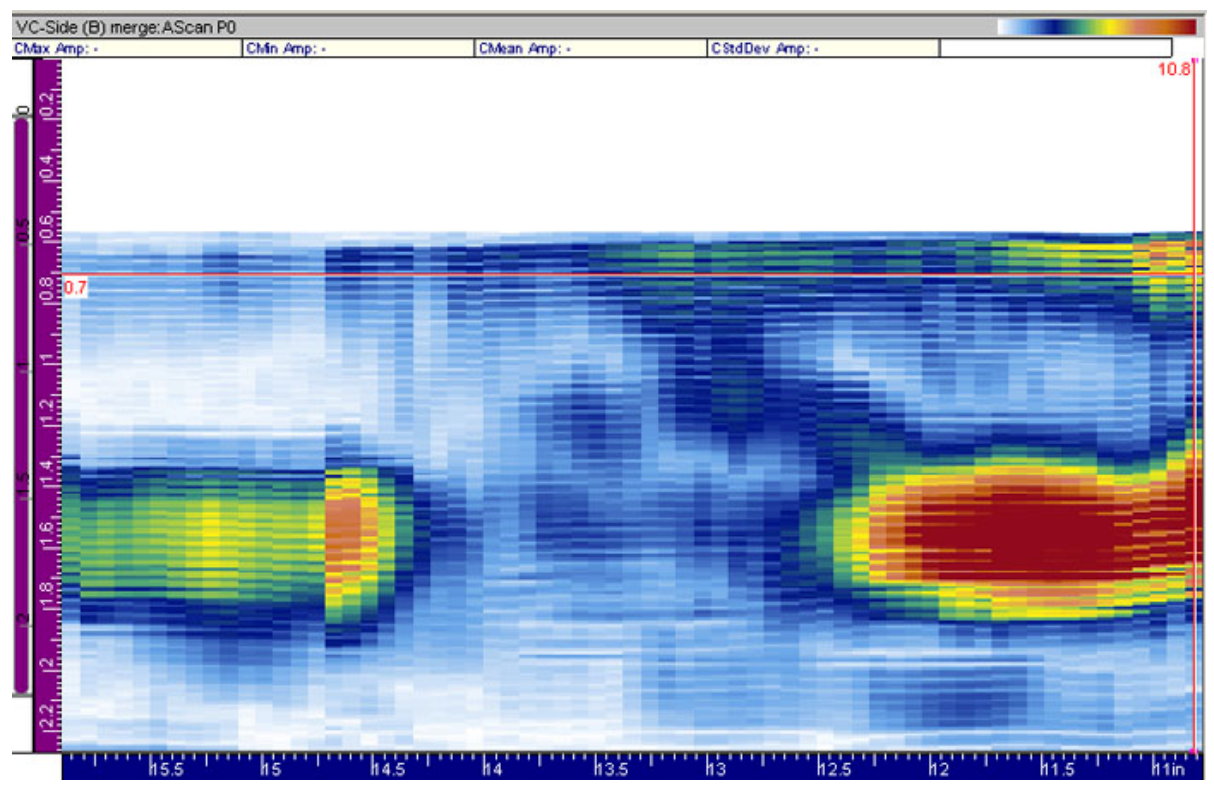

Figure D.5. Pressurizer Surge Line, Flaw 2

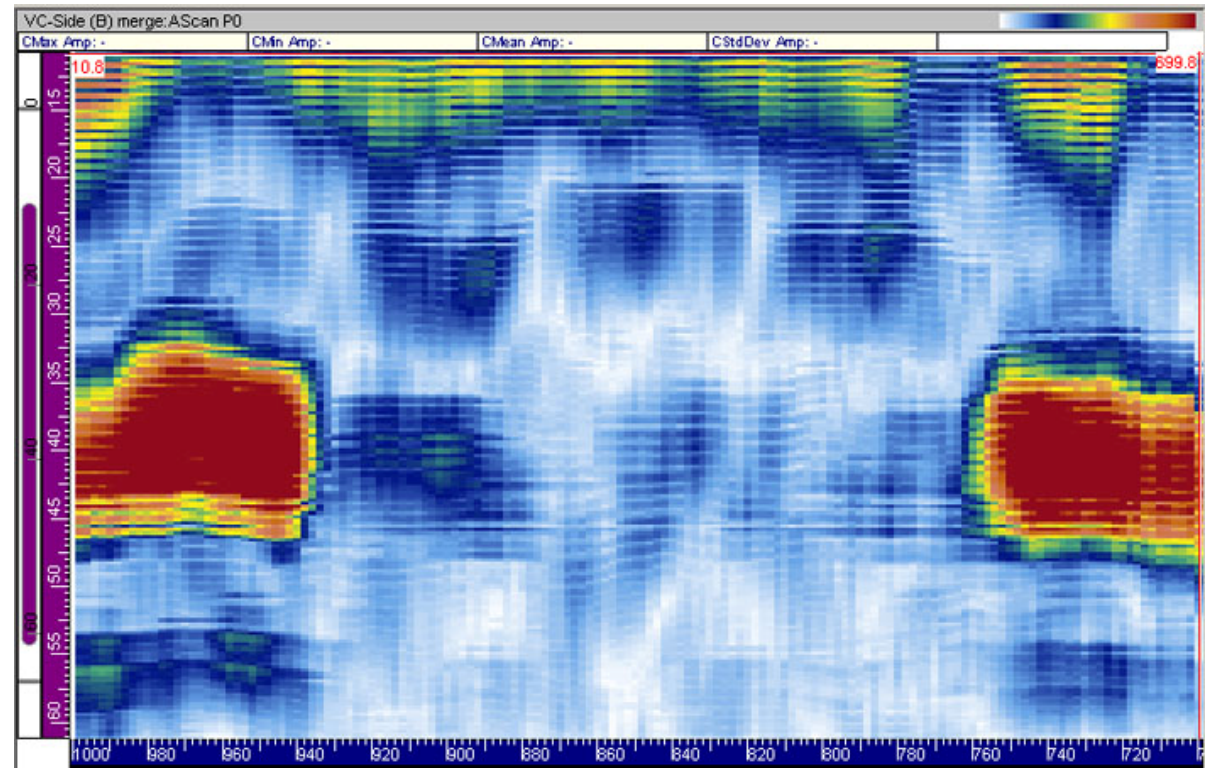

Figure D.6. Pressurizer Surge Line, Flaw 4 


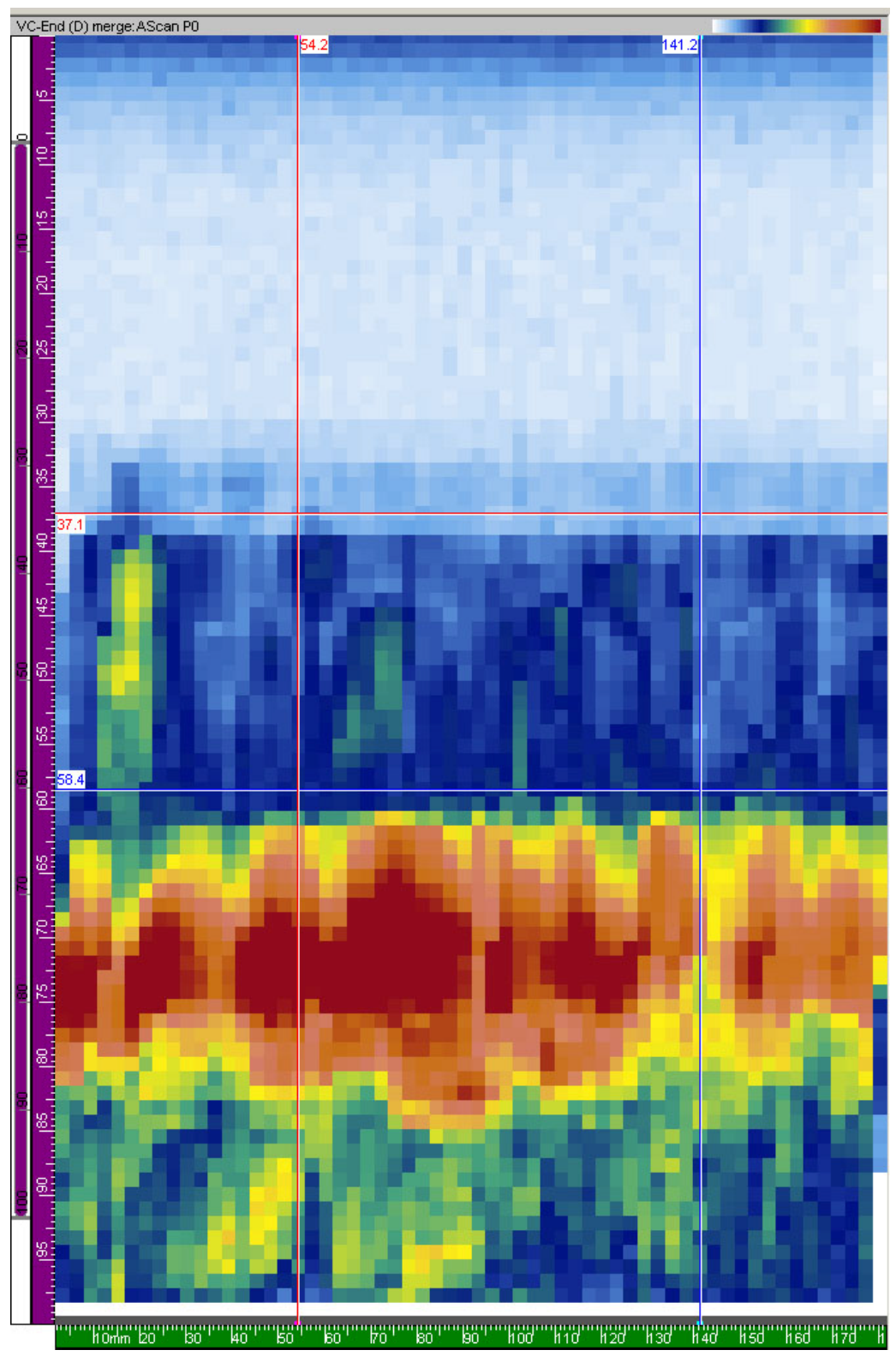

Figure D.7. OPE-5, 52 ${ }^{\circ}$ Transmit, 0.6-inch Separation, 15-mm Focal Plane 


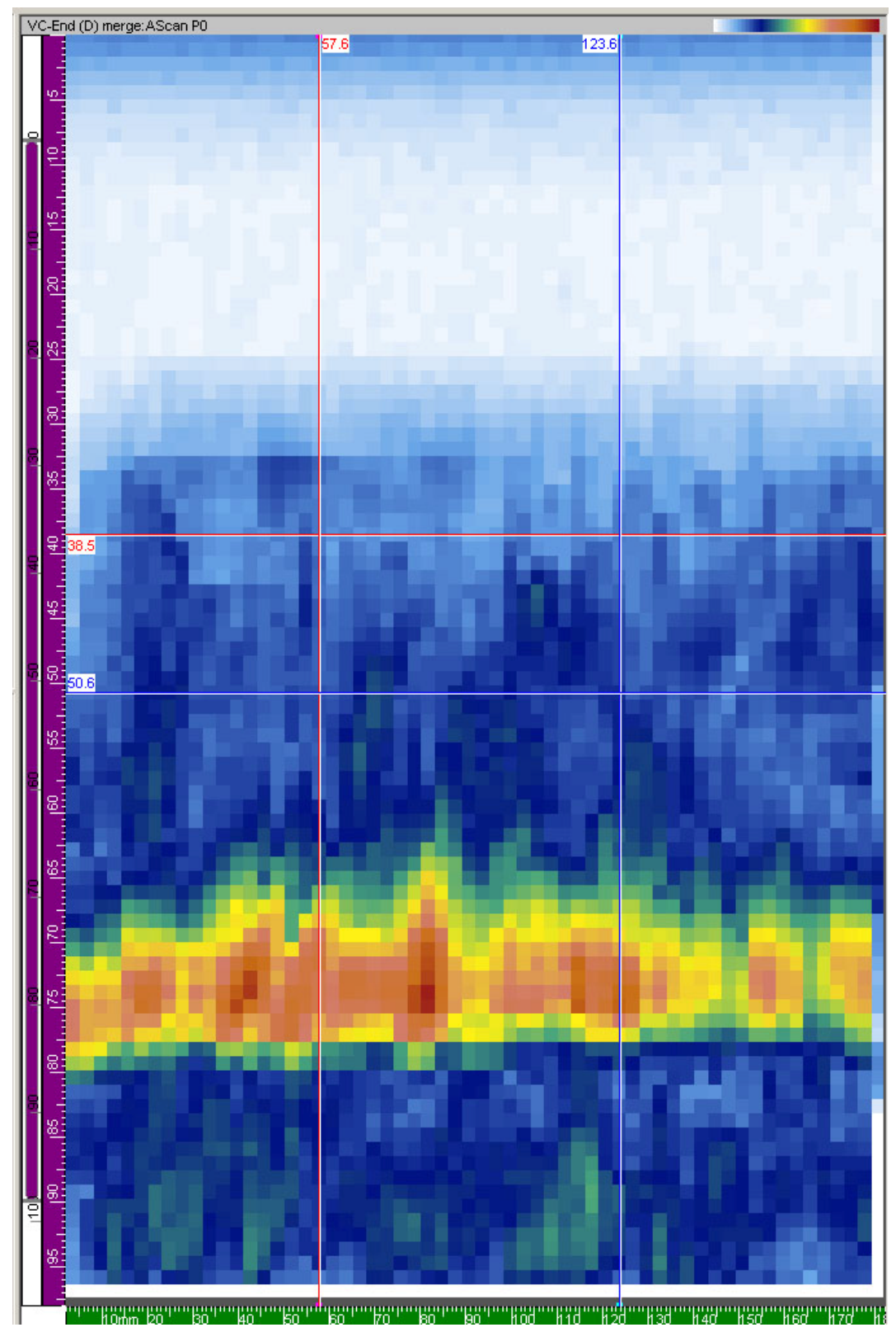

Figure D.8. OPE-5, 55 ${ }^{\circ}$ Transmit, 0.65-inch Separation, 15-mm Focal Plane 


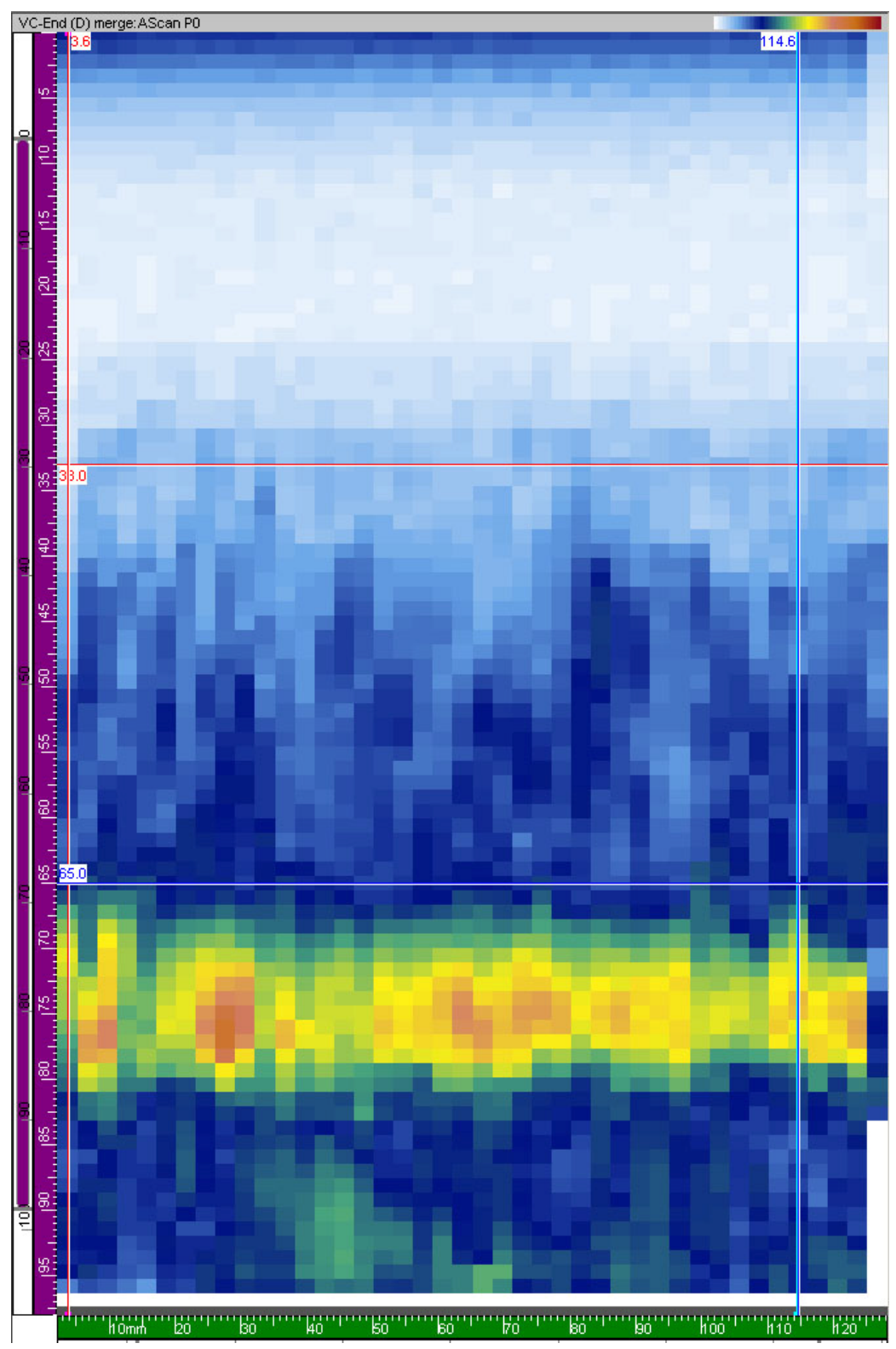

Figure D9. PNNL CASS Specimen B-515, 55 Transmit, 0.65-inch Separation, 15-mm Focal Plane 



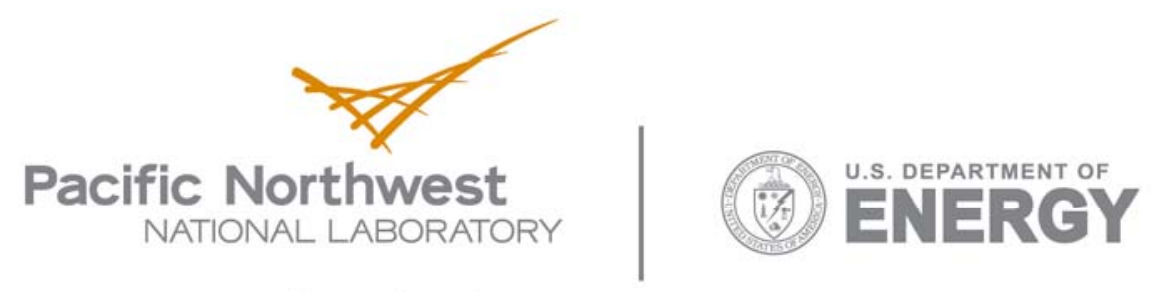

902 Battelle Boulevard

P.O. Box 999

Richland, WA 99352

1-888-375-PNNL (7665)

www.pnl.gov 